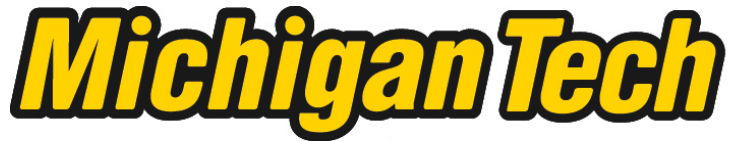 \\ Michigan Technological University Create the Future Digital Commons @ Michigan Tech
}

Dissertations, Master's Theses and Master's Reports - Open

Dissertations, Master's Theses and Master's

Reports

2012

\section{Arc-flash analysis of utility power systems}

Kevin A. Demeny

Michigan Technological University

Follow this and additional works at: https://digitalcommons.mtu.edu/etds

Part of the Electrical and Computer Engineering Commons

Copyright 2012 Kevin A. Demeny

\section{Recommended Citation}

Demeny, Kevin A., "Arc-flash analysis of utility power systems", Master's report, Michigan Technological University, 2012.

https://doi.org/10.37099/mtu.dc.etds/574

Follow this and additional works at: https://digitalcommons.mtu.edu/etds

Part of the Electrical and Computer Engineering Commons 


\title{
ARC-FLASH ANALYSIS OF UTILITY POWER SYSTEMS
}

\author{
By \\ Kevin A. Demeny
}

\begin{abstract}
A REPORT
Submitted in partial fulfillment of the requirements for the degree of MASTER OF SCIENCE ELECTRICAL ENGINEERING
\end{abstract}

\section{MICHIGAN TECHNOLOGICAL UNIVERSITY}

2012

(C) 2012 Kevin A. Demeny 
This report, "Arc-Flash Analysis of Utility Power Systems," is hereby approved in partial fulfillment of the requirements of the Degree of Master of Science in Electrical Engineering.

Department of Electrical and Computer Engineering

Signatures:

Report Advisor:

Dr. Bruce A. Mork

Department Chair:

Dr. Daniel R. Fuhrmann

Date: 


\section{Table of Contents}

Table of Contents............................................................................................... ii

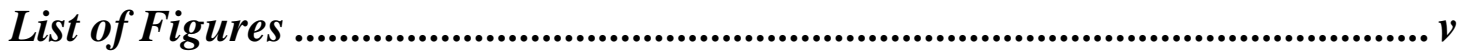

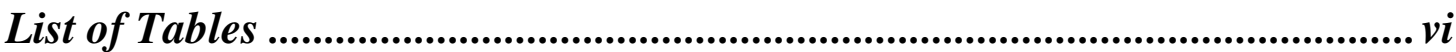

Acknowledgements ....................................................................................................... vii

Abstract ........................................................................................................... vii

Chapter 1: Introduction ...................................................................................... 1

Chapter 2: Background Information and Preexisting Work .............................4

$2.1 \quad$ Literature Search .................................................................................................

2.2 History of Work and Theoretical Foundations .............................................5

2.3 Behavior of Ares..............................................................................

Chapter 3:Arc-Flash Calculation Methods ........................................................ 10

3.1 Applicable Standards......................................................................................... 10

$3.2 \quad$ Emperical Equations ....................................................................................... 11

3.3 National Electrical Safety Code Look-up Tables........................................ 15

$3.4 \quad$ ASPEN ${ }^{\circ}$ Software .................................................................................. 18

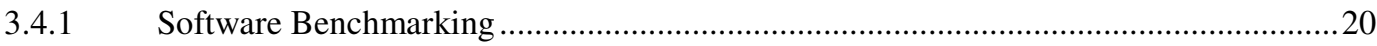

Chapter 4: Issues of Concern ...........................................................................22

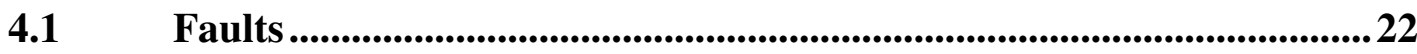

4.2 Energy Exposure..........................................................................................23

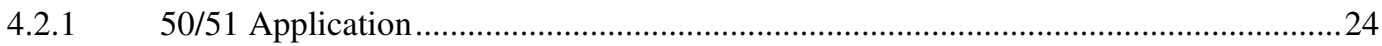

4.2.2 Discontinuity Due to Voltage Levels ............................................................2 24

4.3 Linearity of Incident Energy ..........................................................................33

4.4 Radial Feeders.................................................................................................35

Chapter 5:Mitigation Methods and Hazard Reduction .......................................37

5.1 System Modifications..........................................................................................37

5.2 Relay Application Changes .............................................................................37

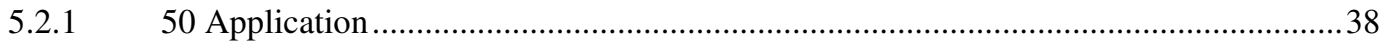

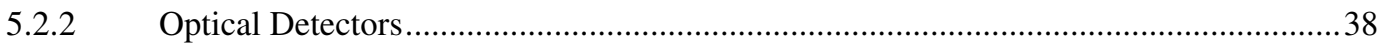


Chapter 6: Conclusions and Recommendations................................................39

6.1 Observations and Conclusions...............................................................39

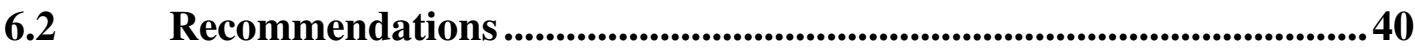

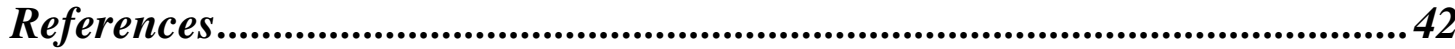

Appendix A Procedure for Arc-Flash Analysis .................................................43

Appendix B Arc-Flash Analyzer Verification Tools............................................45

B.1 Microsoft Excel Verification Tool ................................................................45

B.2 Microsoft Excel Verification Tool with Equations ...................................46

B.3 MATLAB Verification Tool...............................................................47

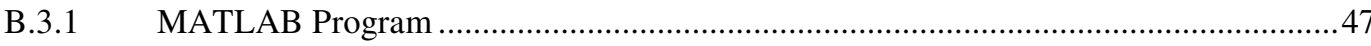

Appendix C Example System Data .......................................................................57

C.1 System Online Diagram..................................................................................57

C.2 Generator Data.......................................................................................57

C.3 Load Data ...............................................................................................................58

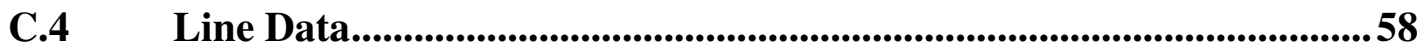

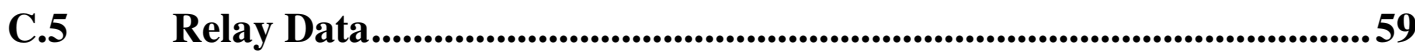

Appendix D Example System ASPEN囚 Output Data .....................................60

D.1 ASPEN ${ }^{2}$ Results .......................................................................................60

D.2 PPE Levels Required ......................................................................................65

Appendix E Discontinuity of Voltage Levels........................................................66

E.1 Voltage Level Test System..............................................................................66

E.2 ASPEN ${ }^{2}$ Results System 1, 51 Contact .......................................................66

E.3 ASPEN $®$ Results System 1, 50 Contact ......................................................67

E.4 ASPEN ${ }^{\circ}$ Results System 2, 51 Contact ......................................................68

E.5 ASPEN ${ }^{\circ}$ Results Using 50 and 51 Contacts........................................69

E.6 ASPEN ${ }^{2}$ Results Systems $1 \& 2$....................................................................70

Appendix F Energy Plot Data........................................................................... 71 


\section{List of Figures}

Figure 1.1: Lab demonstration of arc-flash explosion............................................. 1

Figure 2.1: Vector diagram of arc drop as arc length is varied ................................. 8

Figure 3.1: ASPEN@ with bus selected to run arc-flash calculator ........................... 18

Figure 3.2: ASPEN@ arc-flash calculator displayed ............................................... 19

Figure 3.3: ASPEN® arc-flash hazard calculator user interface .............................. 20

Figure 4.1: Example system oneline diagram ........................................................ 23

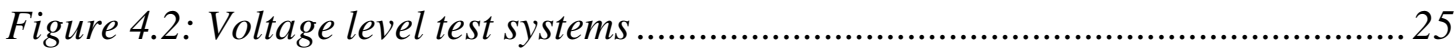

Figure 4.6: Incident energy based on empirical equations only...............................2 29

Figure 4.7: Incident energy based on Lee equation only ........................................ 30

Figure 4.8: Incident energy following given bus voltage .......................................... 31

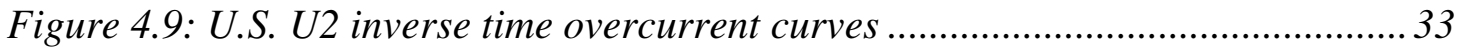

Figure 4.10: Energy through equipment as current increases.................................. 34

Figure 4.11: Potential incident energy in equipment as current increases ................. 35

Figure B.1: MATLAB verification tool user interface …......................................... 47

Figure C.1: Example system oneline diagram ...................................................... 57

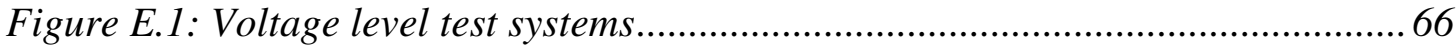




\section{List of Tables}

Table 3.1: Factors for equipment and voltage class ........................................... 12

Table 3.2: PPE required given equipment voltage, fault current and clearing time ... 15

Table 3.3: Live-line tool work PPE required given equipment voltage, fault current and clearing time ............................................................................... 17

Table 4.1: $1 \mathrm{kV}$ voltage level test system incident energy values .............................. 32

Table 4.2: $15 \mathrm{kV}$ voltage level test system incident energy values ............................ 32

Table B.1: Arc-flash hazard calculator................................................................ 45

Table B.2: Arc-flash hazard calculator with equations ........................................... 46

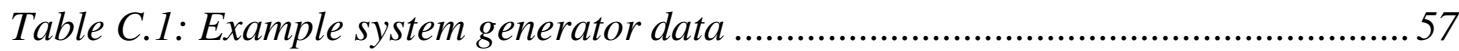

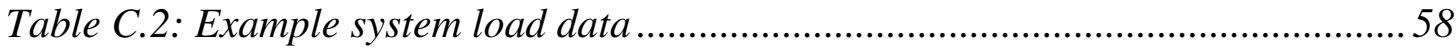

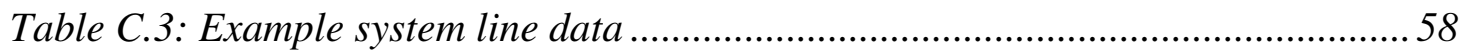

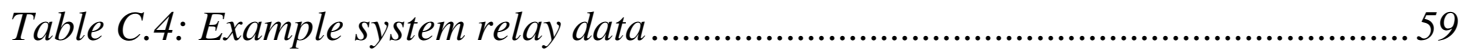

Table D.1: Example system PPE level requirements ............................................ 65

Table E.1: Incident energy level based on trip contact selected ..............................69

Table E.2: Voltage level system comparisons ................................................... 70

Table F.1: U.S. U2 inverse time overcurrent curve data ........................................ 71

Table F.2: Energy through equipment curve data ................................................ 73

Figure F.3: Potential incident energy in equipment curve data .............................. 83 


\section{Acknowledgements}

I would like to thank my advisor, Dr. Bruce Mork, for is guidance, support, time and patience during my experience with this project. I would also like to thank Tom Ernst for his knowledge and support while working under his guidance at Minnesota Power over the summer of 2008. I would also like to thank both of them for considering me for this opportunity which provided me many additional skills in addition to my classroom opportunities along with financial support.

A special thanks to the committee members for taking time to review my work and providing their professional guidance.

I would like to thank, not only the faculty and staff in the Electrical Engineering department, but all faculty and staff at Michigan Tech for their guidance and support during my many years at this great school.

I would like to thank my parents Mr. Martin Demeny and Mrs. Yvonne Heller for their moral support and motivation; without which I wouldn't be who I am and where I am today. I would also like to thank my brothers, Kyle Demeny and Kelly Demeny, along with the rest of my family for their support over the years.

I want to dedicate this work to my parents who deserve all the credit for my success. 


\begin{abstract}
The electric utility business is an inherently dangerous area to work in with employees exposed to many potential hazards daily. One such hazard is an arc flash. An arc flash is a rapid release of energy, referred to as incident energy, caused by an electric arc. Due to the random nature and occurrence of an arc flash, one can only prepare and minimize the extent of harm to themself, other employees and damage to equipment due to such a violent event.

Effective January 1, 2009 the National Electric Safety Code (NESC) requires that an arc-flash assessment be performed by companies whose employees work on or near energized equipment to determine the potential exposure to an electric arc.

To comply with the NESC requirement, Minnesota Power's (MP's) current short circuit and relay coordination software package, ASPEN OneLiner ${ }^{\mathrm{TM}}$ and one of the first software packages to implement an arc-flash module, is used to conduct an arc-flash hazard analysis. At the same time, the package is benchmarked against equations provided in the IEEE Std. 1584-2002 and ultimately used to determine the incident energy levels on the MP transmission system.

This report goes into the depth of the history of arc-flash hazards, analysis methods, both software and empirical derived equations, issues of concern with calculation methods and the work conducted at MP. This work also produced two offline software products to conduct and verify an offline arc-flash hazard analysis.
\end{abstract}




\section{Chapter 1: Introduction}

Electric utility equipment, like any other product we use in everyday activities, is prone to failure and requires preventative maintenance and repair. During these maintenance activities, employees become exposed to a potential arc-flash incident. An arc-flash incident is a violent event exposing employees to not only high temperatures and noise levels but also shrapnel from the equipment involved in the arc flash. This incident may be caused by the employee themselves or by a random equipment failure on nearby equipment.

Effective January $1^{\text {st }}, 2009$ NESC requires that an arc-flash assessment be performed by companies whose employees work on or near energized equipment to determine the potential exposure to an electric arc employers are. For this reason detailed research has been conducted on the behavior of arc flashes and emperical equations have been developed to predict the energy released during the event and IEEE Std. 1584-2002 enforces the equations. Figure 1.1 below captures what an employee may experience if subjected to an arc-flash incident [1].

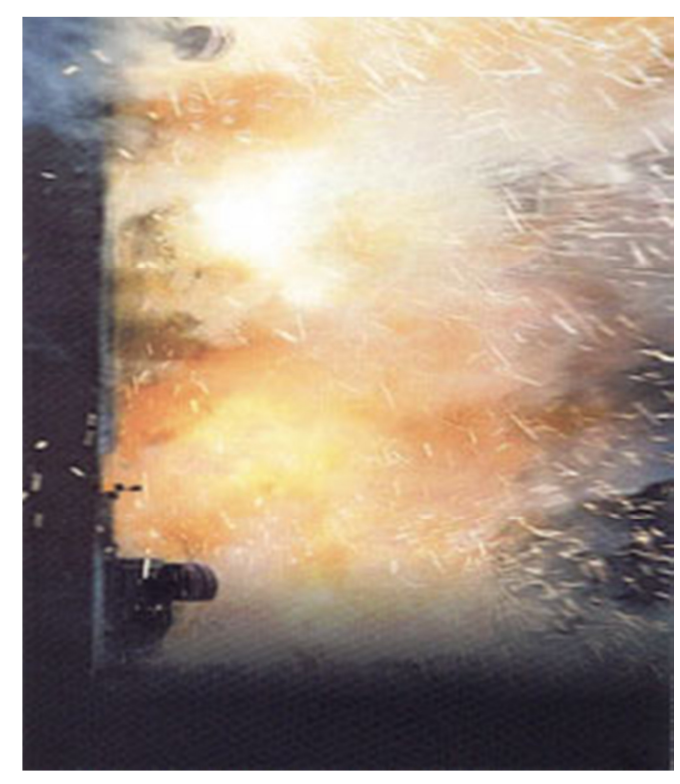

Figure 1.1: Lab demonstration of arc-flash explosion 
Arc-flash assessments are not easy studies to conduct. These studies require both knowledge of the power system and the protective devices used on the system. The effort required to conduct a full system analysis has been greatly reduced with the integration of arc-flash analysis tools in present short circuit software in coordination with protective device data.

The largest effort required in an arc-flash assessment is data collection. There are many variables required as inputs into the detailed equations developed. Some of these variables are also used in short circuit analyses and are readily available while some require engineering judgment to determine which variable to use. The arc energy could be calculated by hand but this also requires a large effort and close attention when entering the data. Several short circuit software programs have implemented an arc-flash analysis module including ASPEN OneLiner ${ }^{\mathrm{TM}}$ which was used to conduct this system assessment.

ASPEN OneLiner ${ }^{\mathrm{TM}}$ is a short circuit and relay coordination program used by protection engineers to simulate different types of faults on a transmission system. Engineers can make changes to relay settings and the configuration of the system and see the effects without needing to re-calculate time consuming short-circuit equations by hand.

One of the objectives of this project is to benchmark the newly released ASPEN $®$ arc-flash module to verify the equations programmed performed to the IEEE Std. 1584-2002.

A second objective of the project is to perform MP's transmission system arcflash assessment to ensure that the company was in compliance with the NESC standards.

Chapter 2 discusses the history of arc-flash hazards, pre-existing work to develop the empirical equations used to calculate the incident energy released during the arc flash, and the behavior of arcs.

Chapter 3 explains the different ways to determine the potential incident energy at a location on the system by using the empirical equations derived in Chapter 
2, by using tables developed by the NESC based on system parameters or by using the ASPEN ${ }^{\circ}$ arc-flash module. Also it details the methodology of benchmarking the software package against the empirical equations.

Chapter 4 provides issues of concerns with the empirical equations based on fault type and the faulted equipment. This chapter also discusses the level of energy exposure due to protective coordination and the voltage level at the fault location.

Chapter 5 will outline potential mitigation methods to be implemented to reduce the hazard exposure with system modifications during maintenance, applying state of the art relay applications or special operating procedures.

Chapter 6 provides conclusions and recommendation for future work. 


\section{Chapter 2: Background Information and Preexisting Work}

This chapter provides background information, preexisting work and the history of arc-flash hazards and analysis.

\subsection{Literature Search}

An arc-flash hazard, as defined by IEEE Std. 1584-2002, is a dangerous condition associated with the release of energy caused by an electric arc. The release of energy is caused by an electric current passing through air between ungrounded conductors or between ungrounded conductors and grounded conductors which is capable of producing temperatures of $35,000^{\circ} \mathrm{F}$. Exposure to these temperatures can burn human skin and ignite the clothing, adding to the burn injury. These high temperatures also cause the explosive expansion of both the surrounding air and the metal in the arc path.

Copper, a metal commonly used in electric equipment, expands by a factor of 67,000 times when it turns from a solid to a vapor. The expansion of the air and metal can cause high pressures, intense sound, and flying shrapnel. The high pressures can exceed hundreds or even thousands of pounds per square foot, possibly knocking workers off ladders, rupturing eardrums, and collapsing lungs. The sounds associated with these pressures can exceed $160 \mathrm{~dB}$ resulting in hearing damage. The shrapnel and molten metal will be expelled away from the arc at speeds exceeding 700 miles per hour which is fast enough to completely penetrate the human body. Each year at least 2,000 people in the U.S. are admitted to burn centers with severe arc-flash burns. Arc flashes can and do kill at distances of 10 feet [2].

According to the NESC 2007 edition Part 4, effective January 1, 2009 employers shall ensure that an assessment is performed to determine potential exposure to an electric arc for employees who work on or near energized parts or equipment. The assessment is to determine the potential incident energy levels to which employees could be exposed while performing work on energized equipment. 
If the assessment reveals that the potential exposure is greater than $2 \mathrm{cal} / \mathrm{cm}^{2}$ at the distance the employee is working from the energized parts $\left(1.2 \mathrm{cal} / \mathrm{cm}^{2}\right.$ is equivalent to the onset of a second degree burn) the employer shall require the employee to wear clothing or a clothing system that has an effective arc rating not less than the anticipated level of arc energy.

When an arc-flash hazard analysis is performed, it shall include a calculation of the estimated arc energy based on the available fault current, the duration of the arc (cycles), and the distance from the arc to the employee. Two exceptions to the analysis are listed below [3]. The clothing or clothing system level required to be worn based on the potential energy released will be discussed in the Chapter 3.

EXCEPTION 1: If the clothing required by this rule has the potential to create additional and greater hazards than the possible exposure to the heat energy of the electric arc, then clothing with an arc rating or arc thermal performance value (ATPV) less than that required by the rule can be worn.

EXCEPTION 2: For secondary systems below $1000 \mathrm{~V}$, applicable work rules required by this part and engineering controls shall be utilized to limit exposure. In lieu of performing an arc-flash hazard analysis, clothing or a clothing system with a minimum effective arc rating of $4 \mathrm{cal} / \mathrm{cm}^{2}$ shall be required to limit the likelihood of ignition.

\subsection{History of Work and Theoretical Foundations}

Prior to 1982 it was assumed that electric shock was the major risk associated with live electrical work [4]. In 1982, Ralph Lee published a paper, The Other Electrical Hazard, Electric Arc Blast Burns, where he describes the thermal event associated with an electric arc and its effect on the human body. In this paper he defines the $1.2 \mathrm{cal} / \mathrm{cm}^{2}$ "curable burn level" that is still used today and the calculations to determine the curable burn distance for an arc in air. Lee's paper is considered by many people as the first research assessing the hazards associated with arc flashes. In 1987 Lee published a second paper regarding arc-flash hazards, Pressures Developed from Arcs. In this paper he describes the sound and pressure effects of an arc in air. 
He also provides charts to determine the pressure wave forces at distances from an arc based on the fault level [5]. In 1990, the threat of an arc flash was well-established, and OSHA updated 29 CFR-1910 Subpart S to recognize the need for arc-flash safety [4].

Two other papers have been published that look at the energies in arcing faults. The first published in 1997, Testing Update on Protective Clothing and Equipment for Electric Arc Exposure, uses empirical test data to determine the incident energy at distances from a low voltage arcing fault. This was the first paper to address the directional effect of an arc in an enclosure. The second paper published in 2000, Predicting Incident Energy to Better Manage the Electric Arc Hazard on 600-V Power Distribution Systems, provided equations to determine incident energy based on the fault level, working distance and the clearing time for arcs in air and in an enclosure on a 600 volt system

$$
\begin{gathered}
E_{M A}=5271 * D_{A}^{-19593} * t_{A} *\left[0.0016 * F^{2}-0.0076 * F+0.8939\right] \\
E_{M B}=1038.7 * D_{B}^{-1.4738} * t_{A} *\left[0.0093 * F^{2}-0.3453 * F+5.9675\right]
\end{gathered}
$$

where

$$
\begin{aligned}
& \mathrm{E}_{\mathrm{MA}}=\text { incident Energy }\left(\mathrm{cal} / \mathrm{cm}^{2}\right) \text { for an arc in open air } \\
& \mathrm{E}_{\mathrm{MB}} \quad=\text { incident Energy }\left(\mathrm{cal} / \mathrm{cm}^{2}\right) \text { for an arc in a box }(20 \text { in. maximum }) \\
& \mathrm{D}_{\mathrm{A}}, \mathrm{D}_{\mathrm{B}}=\text { distance from the arc in inches } \\
& \mathrm{F} \quad=\text { bolted Fault Current }(\mathrm{kA}) \\
& \mathrm{t}_{\mathrm{A}} \quad=\text { time of arc exposure (seconds) }
\end{aligned}
$$

(2.1) and (2.2) were developed based on test data collected from three-phase arc tests conducted in testing laboratories. These tests were conducted under specific conditions in order to collect different incident energy levels. Curve fit equations were then applied to the data to allow the incident energy of the system to be predicted. As can be seen in the equations, the simple form of energy at a given 
distance, $\mathrm{I}^{2} \mathrm{t} / \mathrm{d}^{3}$, isn't clearly evident. By examining the equations closely each component of the simple energy equation can be seen, even if they aren't in the exact same form. This is due to the empirical equations taking into consideration the electrode spacing, whether the arc is in a box or air and other variables not addressed in the simple energy equation.

The work expressed in this paper was used in the formation of the 2000 edition of NFPA-70E Standard for Electrical Safety Requirements for Employee Workplaces in developing safe work practices in regards to arc-flash hazards. The only limitation was that the equations were only good for low voltage applications. The work also formed a basis for future research that resulted in the publication of IEEE Std. 15842002, IEEE Guide for Performing Arc-Flash Hazard Calculations [5]. The same year that the standard was introduced, the first requirement for arc-flash warning labels appeared in the 2002 version of the National Electric Code (NEC) [4]. Equations developed in the IEEE standard will be addressed in the Chapter 3.

\subsection{Behavior of Arcs}

By flashover or from the introduction of some conductive material, an arc is the flow of current through a path consisting of the vapor of the terminal material. This vapor has substantially higher resistance than the solid metal, to the extent that voltage drop in the arc ranges between 75 and 100 V/in, several thousand times its drop in a solid conductor. Since the inductance of the arc is not appreciably different from that of a solid conductor of the same length, the arc current is substantially resistive in nature. Voltage drop in a faulted large solid or stranded conductor is about 0.5 to $1 \mathrm{~V} / \mathrm{ft}$.

For low voltage circuits, the arc, at 75 to $100 \mathrm{~V} /$ in length, consumes a substantial portion of the available voltage, leaving only the difference between source voltage and arc voltage to force the fault current through the total system impedance, including that of the arc. This is the reason for the stabilization of arc current on $277 / 480$ volt circuits when the arc length is of the order of 4 inches. 
For higher voltages, the arc lengths can be substantially greater, say 1 inch per $100 \mathrm{~V}$ of supply, before the system impedance starts to regulate or limit the fault current. The arc voltage drop and the source voltage drop add in quadrature, the former resistive, and the latter substantially reactive. Thus, the length, or size, of arcs in the higher voltage systems can be greater, so they can readily bridge the gap from energized parts to ground or other polarities with little drop in fault current.

In a bolted fault, there is no arc, so little heat will be generated there. Should there be appreciable resistance at the fault point, temperature there could rise to the melting and boiling points of metal and an arc would be started. The longer the arc becomes, the greater the amount of available system voltage will be consumed in it, so the voltage will be available to overcome the supply impedance and the total current will decrease. This is shown in Figure 2.2 [6].

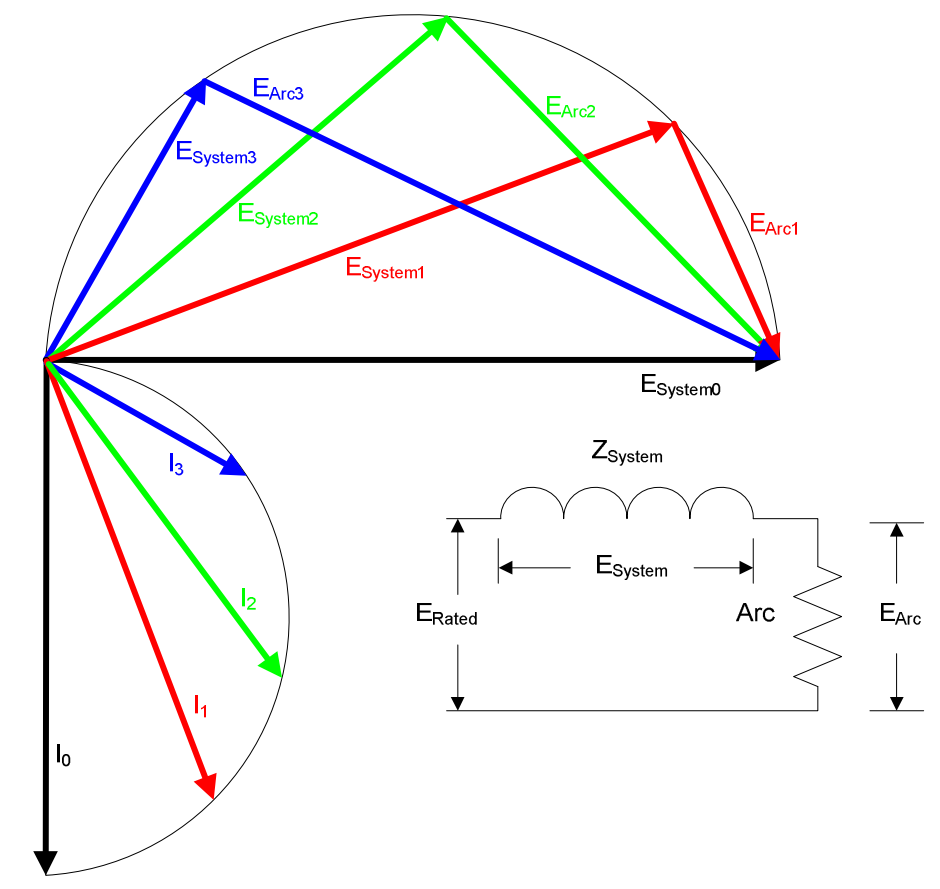

Figure 2.1: Vector diagram of arc drop as arc length is varied

The system has rated voltage $\mathrm{E}_{\mathrm{Rated}}$ and total impedance to the fault of $\mathrm{Z}_{\text {System. }}$. Four arc conditions are shown, one of zero length (bolted fault), one of short length 
(subscript 1), one of moderate length (subscript 2) and one of greater length (subscript 3). Since the arc impedance is almost purely resistive and that of the supply system almost purely inductive, the voltage drop across arc and supply system are out of phase by $90^{\circ}$ for all arc lengths. The locus of the intersection of the vectors of supply voltage drop $\left(\mathrm{E}_{\text {System }}\right)$ and arc voltage drop $\left(\mathrm{E}_{\mathrm{Arc}}\right)$ is a semicircle with diameter of

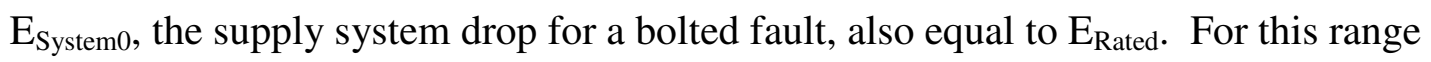
of arc lengths, the total current is represented by current vectors $\mathrm{I}_{0}, \mathrm{I}_{1}, \mathrm{I}_{2}$, and $\mathrm{I}_{3}$, all at right angles to the corresponding $\mathrm{E}_{\text {System. }}$. The magnitude of vectors I are proportional to that of the $E_{\text {System }}$ vectors, since they are related by the constant $Z_{\text {System }}$, ( $I=E_{\text {System }} /$ $\left.\mathrm{Z}_{\text {Sytem }}\right)$.

The total energy in the arc then is the product of $\mathrm{E}_{\mathrm{Arc}}$ and $\mathrm{I}$. This is zero for the bolted fault, appreciable for condition 1 , very substantial for subscript 2 , then decreasing for condition subscript 3 , where the arc voltage increases only moderately while the current decreases substantially. Also, somewhere in the region of subscript 2 to subscript 3 , the length of the arc may become so long that the arc is selfextinguishing or at least self-stabilizing at a low current level. This would be the condition in burndown of 480/277 V buses with wide spacing, where the arc current stabilizes about $1500 \mathrm{~A}$ for 4 inch spacing at $277 \mathrm{~V}$.

It has been found that condition 2, where the arc voltage drop equals the supply system drop, yields the maximum arc wattage condition. Here, the arc voltage drop is 70.7 percent of the supply voltage and the current is 70.7 percent of the bolted fault level. These are in phase, so the product is pure power, even though the system power factor is $45^{\circ}$ lagging at the time, due to the supply system impedance of 0 power factor. Under these conditions the maximum arc wattage is $0.707^{2}$ or 0.5 times the maximum kVA bolted fault capability of the system at that point [6]. 


\section{Chapter 3: Arc-Flash Calculation Methods}

In this chapter, the standards applicable to an arc-flash analysis will be discussed both in the theoretical explanation and how the equations developed to determine incident energy levels are implemented in software packages to be used on large scales on a full power system. Also the benchmarking of the software package used in this project will be discussed.

\subsection{Applicable Standards}

There are a couple different methods proposed to conduct an arc-flash analysis. The two methods addressed and examined with this project are IEEE Std. 1584-2002, Guide for Performing Arc-Flash Hazard Calculations and NESC C2-2007. Each of these will be discussed in detail in this chapter.

When using the IEEE equations to determine the incident energy of a specific point in a system, such as at a bus or switchgear, the equations look and seem overwhelming because of the many variables used which also vary based on the voltage of the system. Along with the many variables there are a few steps that need to be followed in order to calculate the incident energy. These steps are shown below along with the equations needed in each. It should be noted that these equations are not valid for single phase or dc systems. These models are based on measured arc current incident energy under a specific set of test conditions and on theoretical work. These models will enable users to calculate the estimated maximum incident energy and the estimated arc-flash boundary distance. Real arc exposures may be more or less severe than indicated by these models.

The first step that needs to be conducted is to determine the bolted fault current at the point in the system that is being analyzed. The three phase fault current at the location is needed for these calculations. The next step is to determine the voltage level at the point of interest. Voltage levels are broken up into two different categories, less than $1 \mathrm{kV}$ and 1 to $15 \mathrm{kV}$, and based on these ranges different 
equations are used to calculate the arcing current. For voltages less than $1 \mathrm{kV}$ (3.1) is used and for voltages from $1 \mathrm{kV}$ to $15 \mathrm{kV}$ (3.2) is used. Both equations are shown below along with what each of the variables represent. The equation for voltages greater than $15 \mathrm{kV}$ will be discussed later.

\subsection{Emperical Equations}

For applications with a system voltage less than or equal to $1 \mathrm{kV}$,

$$
\begin{gathered}
l_{g} I_{a}=K+0.662 * l_{g} I_{F}+0.0966 * V+0.000526 * G+0.558 * V *\left(l_{g} I_{F}\right)- \\
0.00304 * G *\left(l_{g} I_{F}\right)
\end{gathered}
$$

For applications with a system voltage of 1 to $15 \mathrm{kV}$,

$$
l_{g} I_{a}=0.00402+0.983 * l_{g} I_{F}
$$

where

$$
\begin{array}{ll}
l_{\mathrm{g}} & =\log 10 \\
\mathrm{I}_{\mathrm{a}} & =\operatorname{arcing} \text { current }(\mathrm{kA}) \\
\mathrm{K} & =-0.153 \text { for open configurations (no enclosure) and }-0.097 \text { for box } \\
& \text { configurations (enclosed equipment) } \\
\mathrm{I}_{\mathrm{F}} & =\text { bolted fault current for three-phase faults (symmetrical RMS) (kA) } \\
\mathrm{V} & =\text { system voltages }(\mathrm{kV}) \\
\mathrm{G} & =\text { gap between conductors }(\mathrm{mm})
\end{array}
$$

Table 3-1 provides a list of equipment for the different voltage classes, the typical gap between conductors of that equipment and a distance factor that will be used in later equations. 
Table 3.1: Factors for equipment and voltage class

\begin{tabular}{|l|l|c|c|}
\hline \multirow{4}{*}{ System voltage $(\mathrm{kV})$} & Equipment type & $\begin{array}{c}\text { Typical gap } \\
\text { between } \\
\text { conductors } \\
(\mathrm{mm})\end{array}$ & Distance X factor \\
\hline \multirow{4}{*}{$0.208-1$} & Open air & $10-40$ & 2.000 \\
\cline { 2 - 4 } & Switchgear & 32 & 1.473 \\
\cline { 2 - 4 } & MCC and panels & 25 & 1.641 \\
\cline { 2 - 4 } & Cable & 13 & 2.000 \\
\hline \multirow{3}{*}{ greater than 1 to 5} & Open air & 102 & 2.000 \\
\cline { 2 - 4 } & Switchgear & $13-102$ & 0.973 \\
\cline { 2 - 4 } & Cable & 13 & 2.000 \\
\hline \multirow{3}{*}{ greater than 5 to 15} & Open air & $13-153$ & 2.000 \\
\cline { 2 - 4 } & Switchgear & 153 & 0.973 \\
\cline { 2 - 4 } & Cable & 13 & 2.000 \\
\hline
\end{tabular}

By raising 10 to the power of $1_{\mathrm{g}} \mathrm{I}_{\mathrm{a}}$ in (3.3),

$$
I_{a}=10^{l_{g} I_{a}}
$$

arcing current is determined and can be used in following equations for the incident energy. The next step in the analysis is to calculate the incident energy based on data normalized for an arc of 0.2 seconds and distance from the possible arc point to the person of $610 \mathrm{~mm}$. (3.4) is used in the calculation for both voltage ranges of less than or equal to $1 \mathrm{kV}$ and 1 to $15 \mathrm{kV}$

$$
l_{g} E_{n}=K_{1}+K_{2}+1.081 * l_{g} I_{a}+0.0011 * G
$$

where

$$
\begin{array}{ll}
\mathrm{E}_{\mathrm{n}} & =\text { incident energy }\left(\mathrm{J} / \mathrm{cm}^{2}\right) \text { normalized for time and distance } \\
\mathrm{K}_{1} & =-0.792 \text { for open configurations (no enclosure) and }-0.555 \text { for box } \\
& \text { configurations (enclosed equipment) } \\
\mathrm{K}_{2} \quad=0 \text { for ungrounded and high-resistance grounded systems and }-0.113 & \text { for grounded systems } \\
\mathrm{G} & =\text { gap between conductors }(\mathrm{mm})(\text { Table } 3.1)
\end{array}
$$


By raising 10 to the power of $1_{\mathrm{g}} \mathrm{E}_{\mathrm{n}}$ in (3.5), incident energy can be used in (3.6) to convert from the normalized state.

$$
E_{a}=10^{l_{g} E_{n}}
$$

After calculating the normalized incident energy, it must be converted to $\mathrm{cal} / \mathrm{cm}^{2}$ at the specific working distance and with the devices clearing time. (3.6) is used in the conversion

$$
E=4.184 * C_{f} * E_{a} *\left(\frac{t}{0.2}\right) *\left(\frac{610^{X}}{D^{X}}\right) * 0.24
$$

where

$$
\begin{array}{ll}
\mathrm{E} & =\text { incident energy }\left(\mathrm{cal} / \mathrm{cm}^{2}\right) \\
\mathrm{C}_{\mathrm{f}} & =\text { calculation factor } \\
\mathrm{E}_{\mathrm{a}} & =\text { normalized incident energy } \\
\mathrm{t} & =\text { arcing time (seconds) } \\
\mathrm{D} & =\text { working distance }(\mathrm{mm}) \\
\mathrm{X} & =\text { distance exponent }(\text { Table } 3.1) \\
0.24 & =\text { conversion factor }
\end{array}
$$

These equations are valid for voltages up to $15 \mathrm{kV}$ and arc gaps between 10 and $153 \mathrm{~mm}$. Equations associated with equipment up to $15 \mathrm{kV}$ are based on the lab testing and data used to develop (2.1) and (2.2) and are applied in the IEEE Std. 15842002.

When the voltages are greater than $15 \mathrm{kV}$ or the arc gap is outside of the range, a theoretical equation, called the Lee Equation, is used as shown in (3.7) were the arc current is considered to be equal to the bolted fault current. This equation is also applied in the IEEE Std. 1584-2002. 


$$
E=2.142 * 10^{6} * V * I_{a} *\left(\frac{t}{D^{2}}\right) * 0.24
$$

where

$$
\begin{array}{ll}
\mathrm{E} & =\text { incident energy }\left(\mathrm{cal} / \mathrm{cm}^{2}\right) \\
\mathrm{V} & =\text { system voltage }(\mathrm{kV}) \\
\mathrm{t} & =\text { arcing time }(\text { seconds }) \\
\mathrm{D} & =\text { working distance }(\mathrm{mm}) \\
\mathrm{I}_{\mathrm{a}} & =\text { bolted fault current }(\mathrm{kA})
\end{array}
$$

The next step in the calculations is to determine the flash protection boundary. This is an approach distance at which a worker will receive a second-degree burn if an arc flash would occur; this is the level of $1.2 \mathrm{cal} / \mathrm{cm}^{2}$. (3.8) provides the equation to determine this distance

$$
D_{b}=\left[4.184 * C_{f} * E_{a} *\left(\frac{t}{0.2}\right) *\left(\frac{610^{X}}{E_{b}}\right)\right]^{\frac{1}{X}}
$$

where

$$
\begin{array}{ll}
\mathrm{D}_{\mathrm{b}} & =\text { distance of flash protection boundary from arc (mm) } \\
\mathrm{C}_{\mathrm{f}} & =\text { calculation factor of } 1.5 \text { for voltages less than or equal to } 1 \mathrm{kV} \\
\mathrm{E}_{\mathrm{a}} & =\text { normalized incident energy } \\
\mathrm{E}_{\mathrm{b}} & =\text { incident energy in } \mathrm{J} / \mathrm{cm}^{2} \text { at the flash protection boundary: typically } 5 \\
\mathrm{t} & \mathrm{J} / \mathrm{cm}^{2} \text { which is equal to } 1.2 \mathrm{cal} / \mathrm{cm}^{2} \\
\mathrm{X} & =\text { arcing time (seconds) } \\
610 & =\text { normalance exponent } \\
0.2 & =\text { normalized } 0.2 \text { second clearing time }
\end{array}
$$

(3.8) is based on the current being interrupted by a non-current limiting device. Additional equations are needed if these devices are present. 


\subsection{National Electrical Safety Code Look-up Tables}

The NESC provides look-up tables beginning with the NESC-2007 version of the code. These tables provide arc-flash potentials which can be used instead of performing an arc-flash hazard analysis and provide effective arc rating of clothing or a clothing system to be worn at voltages $1000 \mathrm{~V}$ and above. Table 3.2 provides the level of PPE required based on the fault current and the maximum clearing time of the fault for equipment with voltages from 1 to $46 \mathrm{kV}$.

Table 3.2: PPE required given equipment voltage, fault current and clearing time

\begin{tabular}{|c|c|c|c|c|}
\hline \multirow[b]{2}{*}{$\begin{array}{c}\text { Phase-to-phase } \\
\text { voltage }(\mathrm{kV})\end{array}$} & \multirow[b]{2}{*}{$\begin{array}{l}\text { Fault current } \\
\quad(\mathrm{kA})\end{array}$} & 4-cal System & 8-cal System & 12-cal System \\
\hline & & $\begin{array}{l}\text { Maximum clearing } \\
\text { times in cycles } \\
\text { (seconds) }\end{array}$ & $\begin{array}{l}\text { Maximum clearing } \\
\text { times in cycles } \\
\text { (seconds) }\end{array}$ & $\begin{array}{l}\text { Maximum clearing } \\
\text { times in cycles } \\
\text { (seconds) }\end{array}$ \\
\hline \multirow{4}{*}{1 to 15} & 5 & $46.5(.775)$ & $93(1.55)$ & $139.5(2.325)$ \\
\hline & 10 & $18(.3)$ & $36.1(.6017)$ & $54.1(.9017)$ \\
\hline & 15 & $10(.1667)$ & $20.1(.335)$ & $30.1(.5017)$ \\
\hline & 20 & $6.5(.1083)$ & $13(.2167)$ & $19.5(.325)$ \\
\hline \multirow{4}{*}{15.1 to 25} & 5 & $27.6(.46)$ & $55.2(.92)$ & $82.8(1.38)$ \\
\hline & 10 & $11.4(.19)$ & $22.7(.3783)$ & $34.1(.5683)$ \\
\hline & 15 & $6.6(.11)$ & $13.2(.22)$ & $19.8(.33)$ \\
\hline & 20 & $4.4(.0733)$ & $8.8(.1467)$ & $13.2(.22)$ \\
\hline \multirow{4}{*}{25.1 to 36} & 5 & $20.9(.3483)$ & $41.7(.695)$ & $62.6(1.0433)$ \\
\hline & 10 & $8.8(.1467)$ & $17.6(.2933)$ & $26.5(.4417)$ \\
\hline & 15 & $5.2(.0867)$ & $10.4(.1733)$ & $15.7(.2617)$ \\
\hline & 20 & $3.5(.0583)$ & $7.1(.1183)$ & $10.6(.1766)$ \\
\hline \multirow{4}{*}{36.1 to 46} & 5 & $16.2(.27)$ & $32.4(.54)$ & $48.6(.81)$ \\
\hline & 10 & $7(.1167)$ & $13.9(.2317)$ & $20.9(.3483)$ \\
\hline & 15 & $4.3(0717)$ & $8.5(.1417)$ & $12.8(.2133)$ \\
\hline & 20 & $3(.05)$ & $6.1(.1017)$ & $9.1(.1517)$ \\
\hline
\end{tabular}

This table was built using a commercially available computer software program, not specifically listed in the NESC documentation. Also this table is based on an open air phase-to-ground arc and is not intended to be used for phase-to-phase arcs or enclosed arcs. Assumptions of a 15 inch separation from the arc to the 
employee and arc gaps of 2 inches for 1 to $15 \mathrm{kV}, 4$ inches for 15.1 to $25 \mathrm{kV}, 6$ inches for 25.1 to $36 \mathrm{kV}$ and 9 inches for 36.1 to $46 \mathrm{kV}$ based on the IEEE Standard 4-1995 $[3]$.

Table 3.3 provides the same information except for voltages from 46.1 to 800 $\mathrm{kV}$ was also developed using a computer software program and has its own list of assumptions. First, the air gap was calculated by using the phase-to-ground voltage and dividing by 10 ; this represents the dielectric strength of air at $10 \mathrm{kV}$ per inch. Second, the distance from the arc was calculated by using the minimum approach distance from Table 441-2 in the NESC-2007 and subtracting two times the assumed arc gap length. 
Table 3.3: Live-line tool work PPE required given equipment voltage, fault current and clearing time

\begin{tabular}{|c|c|c|c|c|}
\hline \multirow[b]{2}{*}{$\begin{array}{c}\text { Phase-to-phase } \\
\text { voltage }(\mathrm{kV})\end{array}$} & \multirow[b]{2}{*}{$\begin{array}{l}\text { Fault current } \\
\qquad(\mathrm{kA})\end{array}$} & 4-cal System & 8-cal System & 12-cal System \\
\hline & & $\begin{array}{l}\text { Maximum clearing } \\
\text { times in cycles } \\
\text { (seconds) }\end{array}$ & $\begin{array}{l}\text { Maximum clearing } \\
\text { times in cycles } \\
\text { (seconds) }\end{array}$ & $\begin{array}{l}\text { Maximum clearing } \\
\text { times in cycles } \\
\text { (seconds) }\end{array}$ \\
\hline \multirow{4}{*}{46.1 to 72.5} & 20 & $8.5(.1417)$ & $17(.2833)$ & $25.5(.425)$ \\
\hline & 30 & $5.3(.0883)$ & $10.5(.175)$ & $15.8(.2633)$ \\
\hline & 40 & $3.7(.0617)$ & $7.3(.1217)$ & $11(.1833)$ \\
\hline & 50 & $2.8(.0467)$ & $5.50917)$ & $8.3(.1383)$ \\
\hline \multirow{4}{*}{72.6 to 121} & 20 & $8.2(.1367)$ & $16.5(.275)$ & $24.7(.4117)$ \\
\hline & 30 & $4.7(.0783)$ & $9.4(.1567)$ & $14.1(.235)$ \\
\hline & 40 & $3.1(.0517)$ & $6.2(.1033)$ & $9.3(.155)$ \\
\hline & 50 & $2.2(.0367)$ & $4.4(.0733)$ & $6.6(.11)$ \\
\hline \multirow{4}{*}{138 to 145} & 20 & $9.8(.1633)$ & $19.5(.325)$ & $29.3(.4883)$ \\
\hline & 30 & $5.6(.0933)$ & $11.2(.1867)$ & $16.8(.28)$ \\
\hline & 40 & $3.7(.0617)$ & $7.4(.1233)$ & $11.1(.185)$ \\
\hline & 50 & $2.6(.0433)$ & $5.3(.0883)$ & $7.9(.1317)$ \\
\hline \multirow{4}{*}{161 to 169} & 20 & $9.3(.155)$ & $18.6(.31)$ & $27.9(.465)$ \\
\hline & 30 & $5.7(.095)$ & $11.5(.1917)$ & $17.2(.2867)$ \\
\hline & 40 & $4(.0667)$ & $8(.1333)$ & $12(.2)$ \\
\hline & 50 & $3(.05)$ & $6(.1)$ & $9(.15)$ \\
\hline \multirow{4}{*}{230 to 242} & 20 & $10.4(.1733)$ & $20.9(.3483)$ & $31.3(.5217)$ \\
\hline & 30 & $6.4(.1067)$ & $12.9(.215)$ & $19.3(.3217)$ \\
\hline & 40 & $4.5(.075)$ & $9(.15)$ & $13.5(.225)$ \\
\hline & 50 & $3.4(.0567)$ & $6.8(.1133)$ & $10.1(.1683)$ \\
\hline \multirow{4}{*}{345 to 362} & 20 & $22.6(.3767)$ & $45.3(.755)$ & $67.9(1.1317)$ \\
\hline & 30 & $14(.2333)$ & $28.1(.4683)$ & $42.1(.7017)$ \\
\hline & 40 & $9.8(.1633)$ & $19.6(.3267)$ & $29.4(.49)$ \\
\hline & 50 & $7.4(.1233)$ & $14.7(.245)$ & $22.1(.3683)$ \\
\hline \multirow{4}{*}{500 to 550} & 20 & $18.9(.315)$ & $37.8(.63)$ & $56.7(.945)$ \\
\hline & 30 & $11.7(.195)$ & $23.3(.3883)$ & $35(.5833)$ \\
\hline & 40 & $8.1(.135)$ & $16.3(.2717)$ & $24.4(.4067)$ \\
\hline & 50 & $6.1(.1017)$ & $12.2(.2033)$ & $18.3(.305)$ \\
\hline \multirow{4}{*}{765 to 800} & 20 & $43.6(.7267)$ & $87.3(1.455)$ & $130.9(2.182)$ \\
\hline & 30 & $27(.45)$ & $53.9(.8983)$ & $80.9(1.3483)$ \\
\hline & 40 & $18.9(.315)$ & $37.8(.63)$ & $56.7(.945)$ \\
\hline & 50 & $14.2(.2367)$ & $28.4(.4733)$ & $42.6(.71)$ \\
\hline
\end{tabular}




\subsection{ASPEN® Software}

ASPEN OneLiner ${ }^{\mathrm{TM}}$ was used for this project due to the fact that MP's relay engineers use this product for their short circuit and relay coordination on the power system and is a highly recognized product in the power industry; OneLiner has a $46 \%$ market share according to an independent survey conducted in 2009. Figure 3.1 is a screen shot of an example one line system in ASPEN® selecting a position to run the arc-flash calculator. Figure 3.2 shows the user interface for the arc-flash calculator after it has been selected.

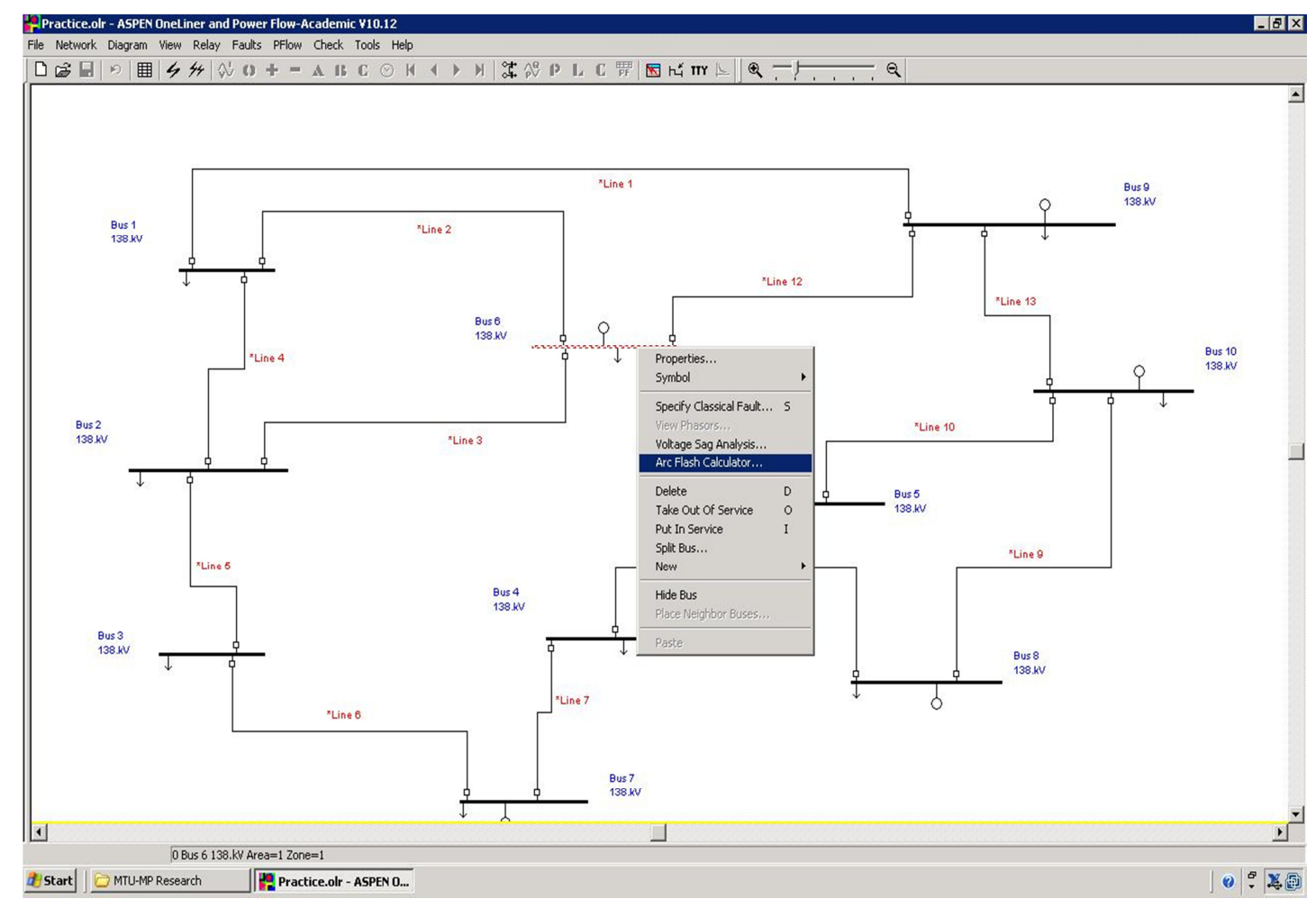

Figure 3.1: ASPEN® with bus selected to run arc-flash calculator 


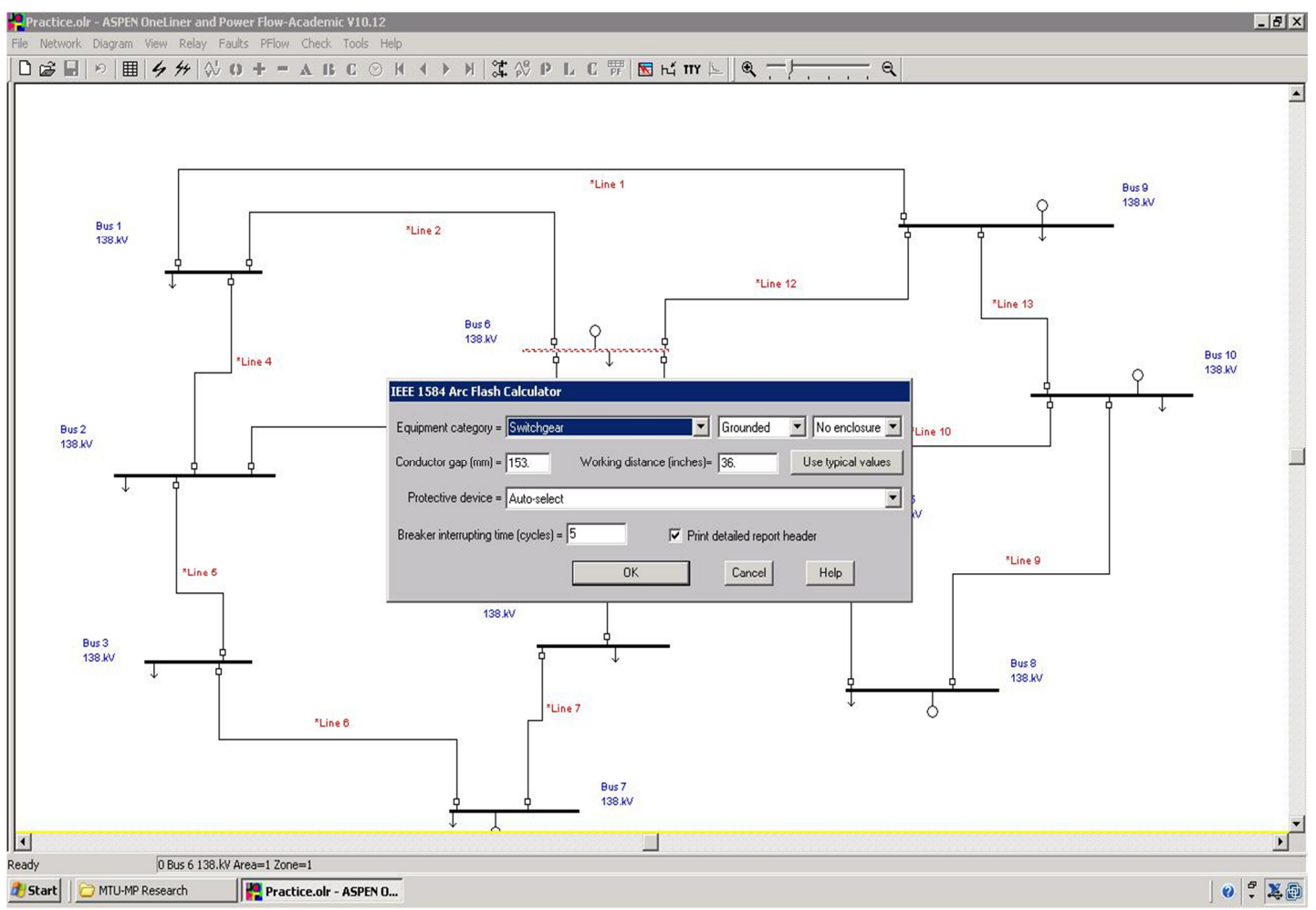

Figure 3.2: ASPEN® arc-flash calculator displayed

Prior to the kick-off of this project ASPEN® had just released their arc-flash calculator which allowed users to carry out the calculation on one equipment location on the system at a time. Figure 3.3 is a zoomed in view of the dialog box user interface with the calculator to enter options about the location of the equipment analyzed. The calculator uses IEEE Std. 1584-2002 equations, explained above in section 3.2, to calculate arcing current and the incident energy level at both $100 \%$ and $85 \%$ of the fault current. The calculation results are output to the TTY window. 


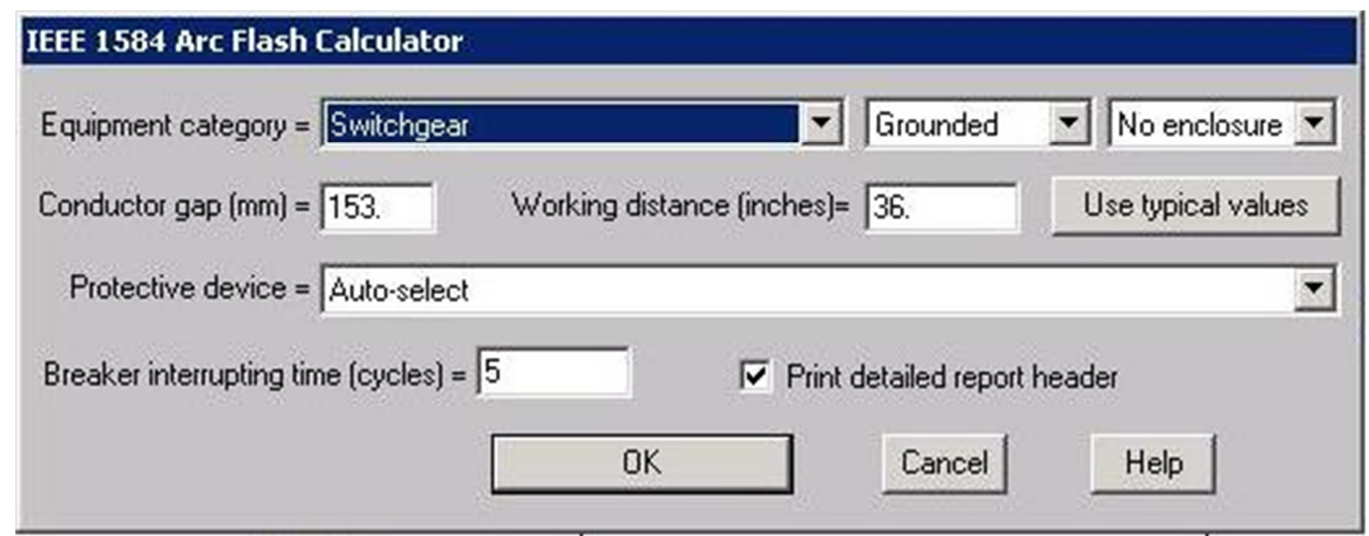

Figure 3.3: ASPEN® arc-flash hazard calculator user interface

\subsubsection{Software Benchmarking}

Since the calculator was released just prior to the project implementation, some benchmarking was recommended to test that the calculator was performing correctly and also to provide some practice in using the interface and reading the results in the TTY window.

Since the ASPEN® calculator uses IEEE Std. 1584-2002 equations, two different offline tools were developed to validate the calculator. The first tool built was an Excel spreadsheet, shown in Appendix B, including the IEEE Std. 1584-2002 equations allowing the user to input values from the ASPEN® calculator and solve for the arc current, normalized incident energy and incident energy. This tool proved to work well but it was quickly realized that the screen space needed to enter inputs, determine which set of equations to use based on voltage and view outputs was rather large.

The second tool built followed the same idea as the Excel spreadsheet but instead was implemented in MATLAB. The user interface and code are shown below in Appendix B. The user interface was greatly reduced and it reduced the chance of the user entering data into the incorrect cell. After the MATLAB calculator was built the equations in the code were verified against hand calculations of the IEEE Std. 1584-2002 equations. 
After the verification of the MATLAB calculator was completed the ASPEN® calculator was tested against the MATLAB calculator based on the test system shown below in Appendix C.

During in-house benchmarking at the university, the ASPEN® calculator performed correctly but it wasn't until the actual MP arc-flash analysis was underway that three software bugs were discovered. The first of these bugs was that reclosers were not recognized as protective devices. This was discovered when clearing times reported were much longer than anticipated based on protective data entered into the power flow model. This bug required a revision to the software code to include these protective devices.

The second bug was the break point for level 2 of incident energy at the equipment location. The IEEE standard has the break point at $4 \mathrm{cal} / \mathrm{cm}^{2}$ while the calculator uses $5 \mathrm{cal} / \mathrm{cm}^{2}$ based on the NFPA 70E-2000.

The third and final bug found was the calculation of the incident energy using $85 \%$ of the arcing current. To ensure the worst case incident energy level is calculated, the ASPEN® calculator also takes $85 \%$ of the arcing current and runs it through the equations. This was discovered when the voltage level of the system was below $15 \mathrm{kV}$. Using these equations, the full short circuit level was being applied when in fact only $85 \%$ of the fault current was to be entered. This was not an issue when the voltage was above $15 \mathrm{kV}$ since only the short circuit current is used to determine the incident energy.

All these issues were brought to ASPEN®'s attention with ASPEN® providing resolution in updated Oneliner.exe files to replace current files on the computers being used to conduct the arc-flash analysis. 


\section{Chapter 4: Issues of Concern}

In this chapter, two areas of concern will be discussed, both of which are main factors affecting the incident energy of an arc flash. The first of these concerns is the type of fault used to conduct an arc-flash analysis. Assumptions of the fault may or may not be correct based on the equipment or location of the fault analyzed. Second, the amount of energy exposure personnel could be exposed could potentially differ based on assumptions of the protective equipment used to clear faults. Each of these points is addressed below.

\subsection{Faults}

During all the research for and implementation of an arc-flash analysis it was determined that there is some discontinuity for the type of fault to be used on different type of equipment. If we look at typical power system there is a correlation to the type of fault personnel could be exposed to depending on the equipment they may be working on. Out in a switch yard working on a $345 \mathrm{kV}$ gang operated disconnect switch, a three phase fault is rarely heard of. On the other hand, a three phase fault inside of some switch gear in a motor control panel is very plausible.

If you look at the IEEE standard a three phase fault current is used based on any voltage level or type of equipment at the point where the analysis is being conducted. For voltage levels up to $15 \mathrm{kV}$ this is justifiable because those equations are based on three phase fault lab test results. With the equation for voltage levels greater than $15 \mathrm{kV}$ it is assumed the fault is a three phase fault. But if you look at Table 3-2 shown above, which is provided by the NESC gives a quick reference lookup table for arc-flash values, a phase to ground fault is used to develop the table. Looking at Table 3-3, NESC doesn't state the type of fault used to develop the table.

An example of this discontinuity can be seen on the test system used to benchmark the ASPEN® software in Figure 4.1. 


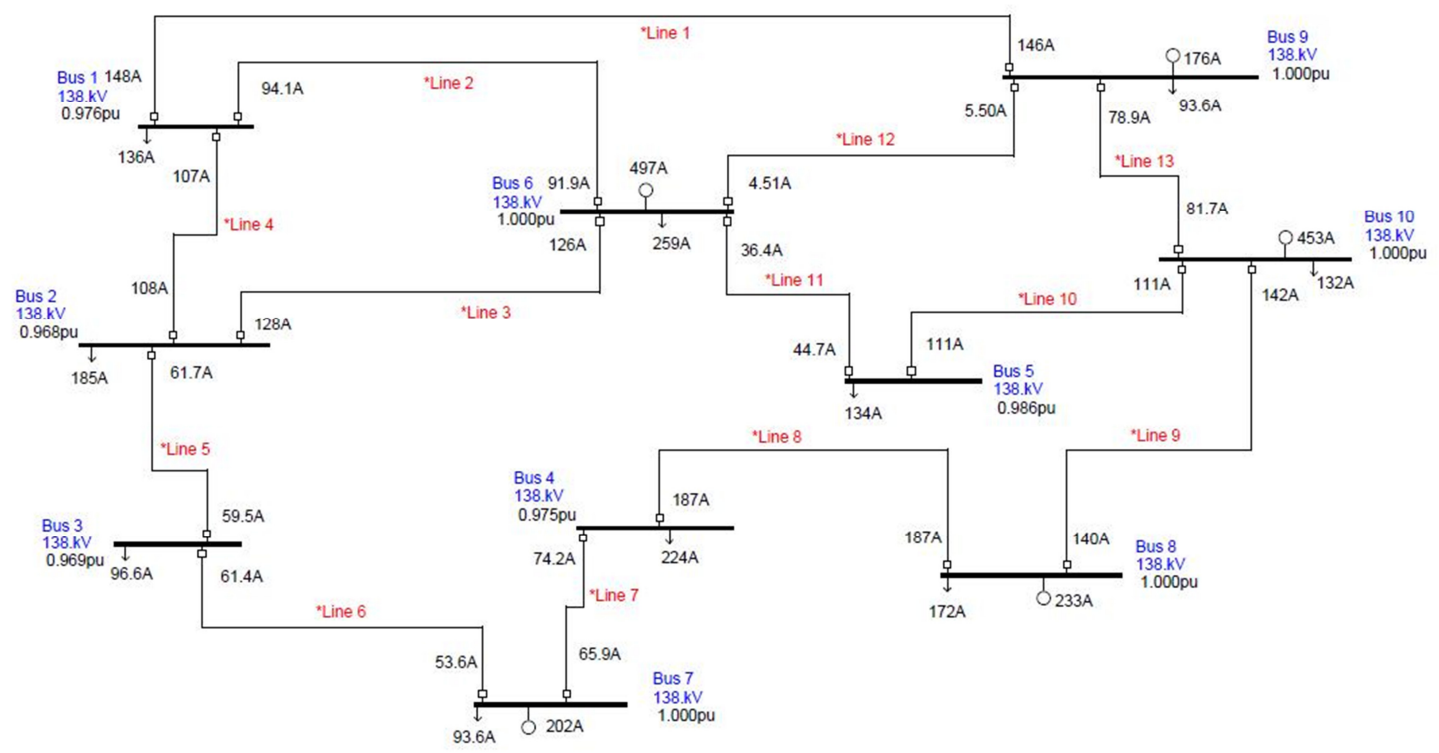

Figure 4.1: Example system oneline diagram

If we look at the $138 \mathrm{kV}$ bus, Bus 1 , in ASPEN®, the three phase fault current is $4.228 \mathrm{kA}$ with a clearing time of 0.11 seconds. These values result in a requirement of PPE level 3. If the NESC table is used, which doesn't state what type of fault to be used, and use the three phase fault current and clearing time from ASPEN®, the table doesn't provide a reference meaning the level is below the minimum 4 calorie system typical substation personnel wear to conduct minor activities, such as a walk around visual inspection.

When it comes to personnel safety, requiring individuals to wear PPE levels 3 and 4 when it isn't required may cause other safety hazards while they perform their assigned tasks. Also, it may cost the company undue expenses in purchasing PPE equipment not really required.

\subsection{Energy Exposure}

When the potential level of incident energy is at or above $40 \mathrm{cal} / \mathrm{cm}^{2}$, the IEEE Std. 1584-2002 says that work cannot be conducted on the energized equipment. Equipment may never be serviced under this requirement and could fail causing further issues on the system. 
The amount of incident energy personnel may become subject to can vary based on two specific variables; the time duration of the fault and the voltage level of the equipment subject to the fault. This chapter will discuss how each of these specific variables affects the potential incident energy.

\subsubsection{0/51 Application}

The application of a 50/51 relay on a bus or line for fault protection uses either an instantaneous trip, the 50 contact, or a time delayed trip based on a relay curve, the 51 contact. Either application is acceptable but choosing one over the other can greatly affect your level of arc-flash hazard present at the fault location. In Appendix E.1, a test system has been built to emphasize this point. In System 1, initially the 51 contact is used to protect both lines of the system. Under this configuration, Bus 2 has an arc-flash category level 2 of $5.5 \mathrm{cal} / \mathrm{cm}^{2}$ and Bus 3 has an arc-flash category level 0 of $1.01 \mathrm{cal} / \mathrm{cm}^{2}$. By using the 50 contact, which will reduce the total clearing time to essentially the time needed to operate the breaker, reduces the Bus 2 and 3 arc-flash categories 0 and $0,0.88$ and $0.62 \mathrm{cal} / \mathrm{cm}^{2}$ respectively. The full details of the four different results can be seen in Appendix E.2 and E.3.

\subsubsection{Discontinuity Due to Voltage Levels}

The second variable to be evaluated is the voltage level of the equipment. As mentioned earlier in section $3.2,1 \mathrm{kV}$ is a break point for which equation to use in calculating the arcing current and $15 \mathrm{kV}$ is a break point at which empirical equations are not to be used in analysis but instead the Lee equation. On a typical transmission or distribution system, voltages can range from 0.95 to 1.05 per unit on an intact system. For a $1 \mathrm{kV}$ system the voltage could run between 0.95 to $1.05 \mathrm{kV}$ and for a 15 $\mathrm{kV}$ system the voltage range could run between 14.25 and $15.75 \mathrm{kV}$. This results in two different set of equations to be considered when evaluating one piece of equipment. If the incorrect equation is used, lower levels of incident energy may be calculated in turn causing lower levels of PPE worn by the employee which may not properly protect the individual for an arc-flash incident. 
An evaluation of this condition was studied on the same test system used for the 50/51 contact evaluation. One system was set to a system voltage of $14.9 \mathrm{kV}$ and the second system was set to a voltage level of $15.1 \mathrm{kV}$ displayed in Figure 4.2. The same condition was applied to a $1 \mathrm{kV}$ system except the low level was set to $0.9 \mathrm{kV}$ and the high to $1.1 \mathrm{kV}$.

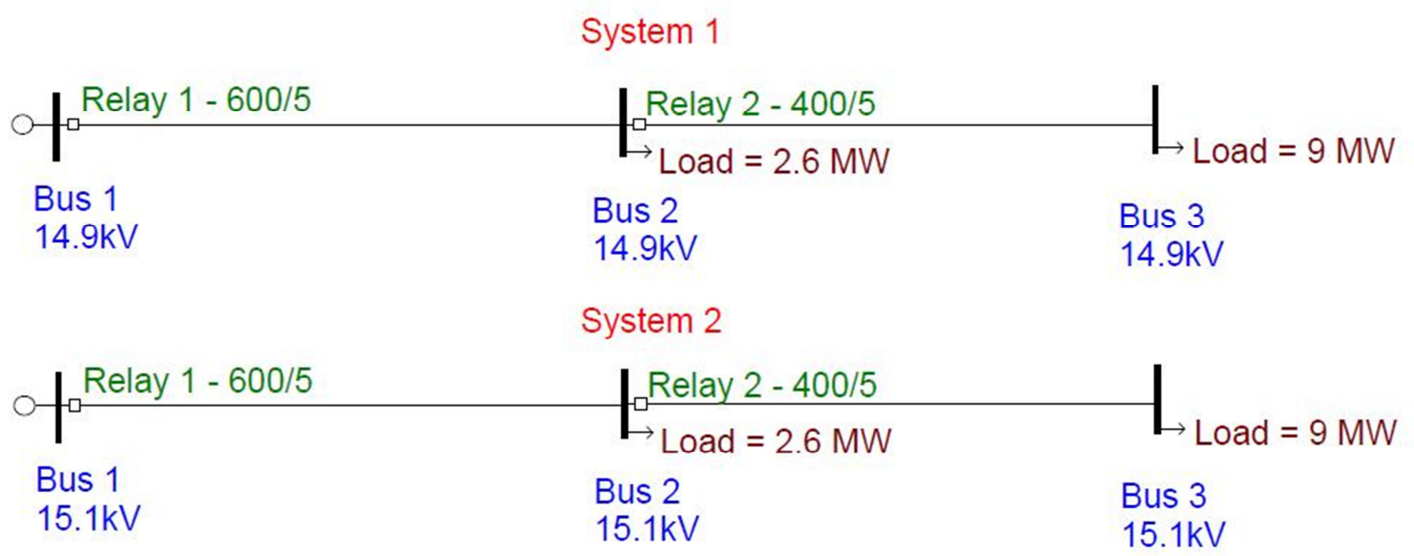

Figure 4.2: Voltage level test systems

This allows both sets of arc-flash equations to be tested with only a 200 volt difference between the two systems. In the field this could easily be found by placing a distribution capacitor into service to boost service voltages or if the employee is working on the piece of equipment under a lighter load timeframe.

As shown in the simulations on the $1 \mathrm{kV}$ system and only using the equation for equipment below $1 \mathrm{kV}$, the incident energy at bus 3 increases exponentially as the voltage increases. This can be seen below in Figure 4.3. 


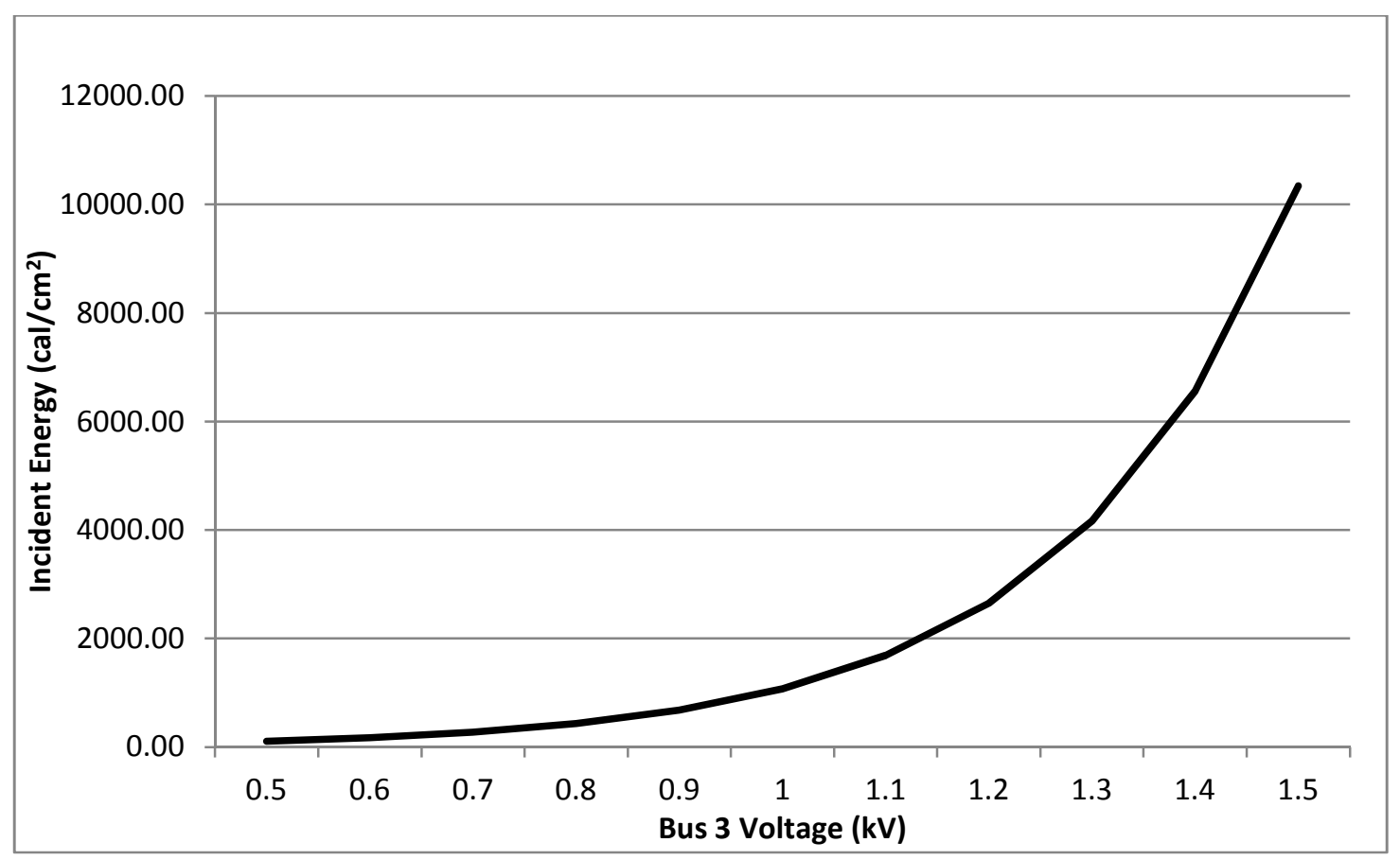

Figure 4.3: Incident energy based on empirical equation for equipment less than $1 \mathrm{kV}$

The opposite trend is found when only using the equation for equipment above $1 \mathrm{kV}$ and less than $15 \mathrm{kV}$. This can be seen below in Figure 4.4. 


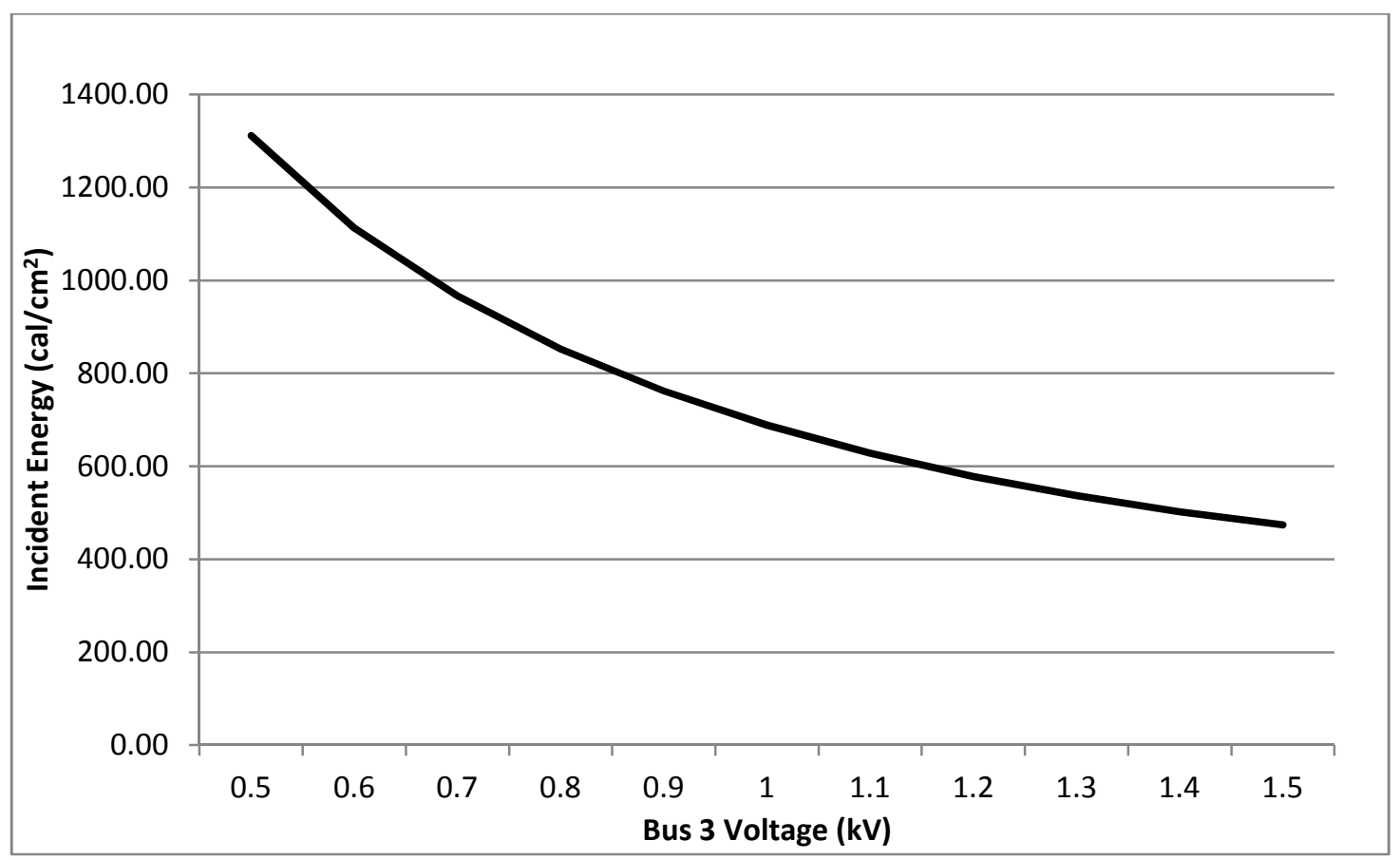

Figure 4.4: Incident energy using equation for equipment above $1 \mathrm{kV}$ and less than $15 \mathrm{kV}$

Figure 4.5 is a plot of the incident energy using the equation required based on the bus voltage. As it shows, the incident energy increases as the voltage increases until it reaches $1 \mathrm{kV}$. At this point, the equation for equipment above $1 \mathrm{kV}$ and less than $15 \mathrm{kV}$ takes over and we see a slight increase in incident energy up to $1.1 \mathrm{kV}$ and then the incident energy decreases. The discontinuity can be associated to two factors.

First, in the equation for equipment less than $1 \mathrm{kV},(3.1)$ above, uses the conductor gap to calculate the arcing current. This full value isn't applied to the equation but a fraction of it is, increasing the log of the arcing current available. This gap isn't applied in the equation for equipment greater than $1 \mathrm{kV}$ and less than $15 \mathrm{kV}$, (3.2) above.

Second, in equation (3.1), the equipment voltage is used to calculate the arcing current. When the voltage is less than $1 \mathrm{kV}$, this applies a fraction of the log of the arcing current. When the log of the arcing current is then applied to find the normalized incident energy, the normalized incident energy is also reduced. Once the voltage is greater than $1 \mathrm{kV}$, the log of the arcing currents starts to decrease causing the decrease in incident energy. An example of this can be seen using the values from 
the test system. When the voltage is at 900 volts, the $1_{g} I_{a}$ is equal to $3.55 \mathrm{kA}$ when using equation (3.1) and $3.76 \mathrm{kA}$ when using equation (3.2). When the voltage is at $1.1 \mathrm{kV}$, the $1_{\mathrm{g}} \mathrm{I}_{\mathrm{a}}$ is equal to $3.92 \mathrm{kA}$ when using equation (3.1) and $3.68 \mathrm{kA}$ when using equation (3.2). If the voltage was to be applied in the equation (3.1), there would be a multiplying factor anywhere from 1.1 to 15 times and the incident energy would continue to increase.

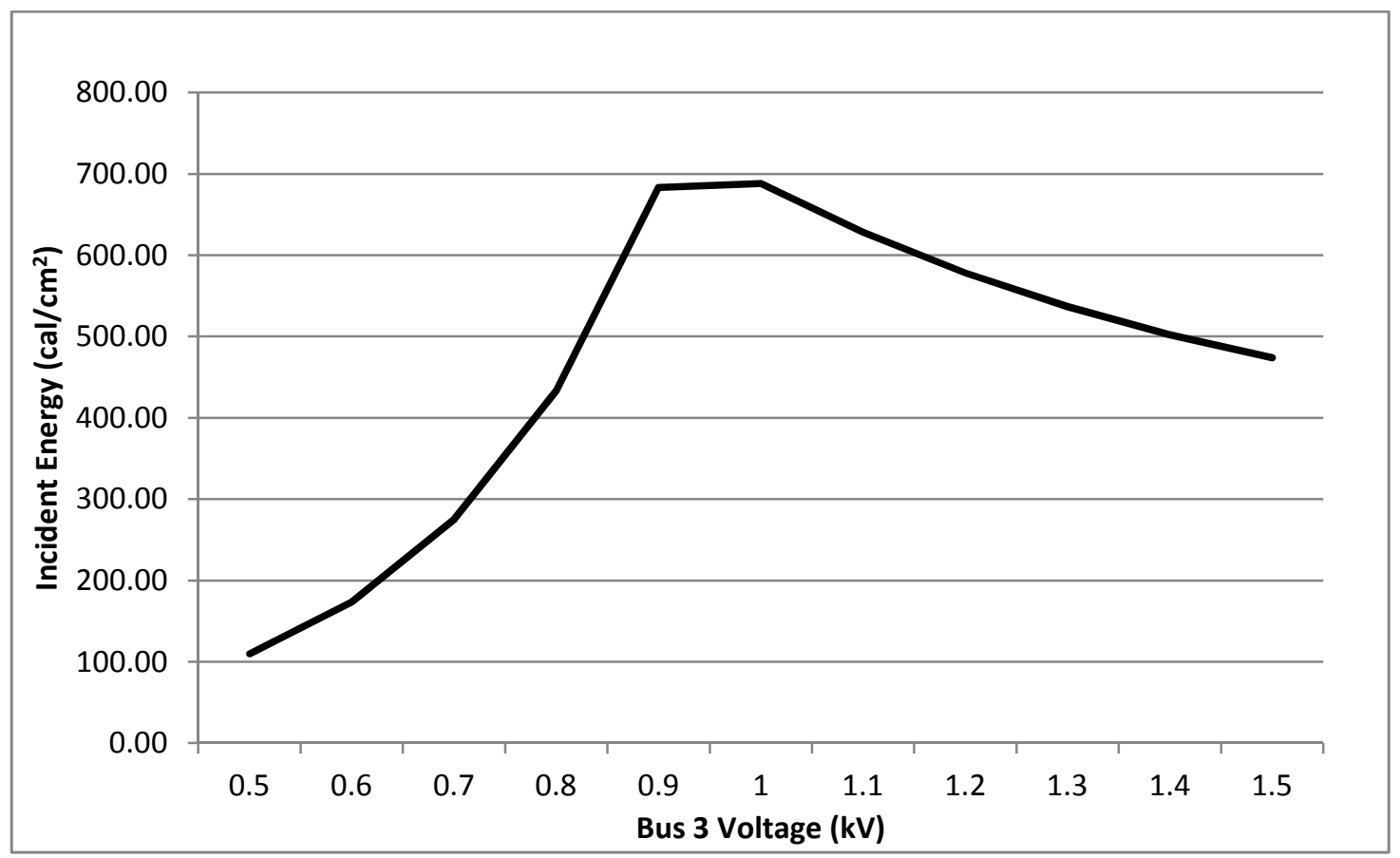

Figure 4.5: Incident energy based on given bus voltage

As shown in the simulation, the incident energy of both buses in the $15.1 \mathrm{kV}$ system increased to levels well above the level found in the $14.9 \mathrm{kV}$ system. For bus 2 the incident energy increased from 5.5 to $130.16 \mathrm{cal} / \mathrm{cm}^{2}$ and bus 3 increased from 1.0 to $25.01 \mathrm{cal} / \mathrm{cm}^{2}$. Figure 4.6 is a plot of the bus 3 incident energy against the bus 3 voltage using the derived equations only based off of lab testing. We see that as the voltage increases the incident energy increases linearly. 


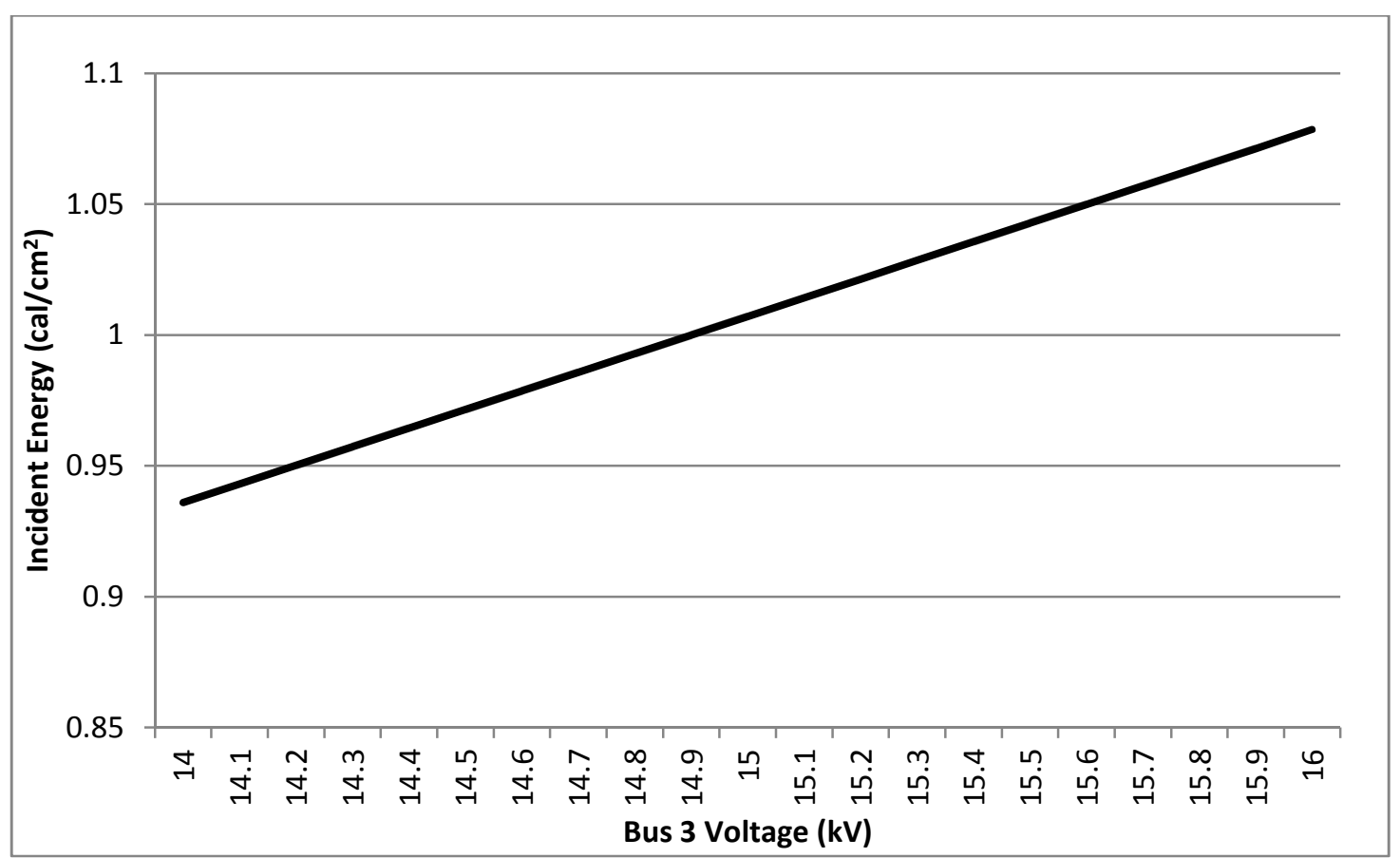

Figure 4.6: Incident energy based on empirical equations only

Figure 4.7 is a plot of the bus 3 incident energy against the bus 3 voltage using the Lee equation only. Again we see the incident energy increase linearly as the bus voltage increases. 


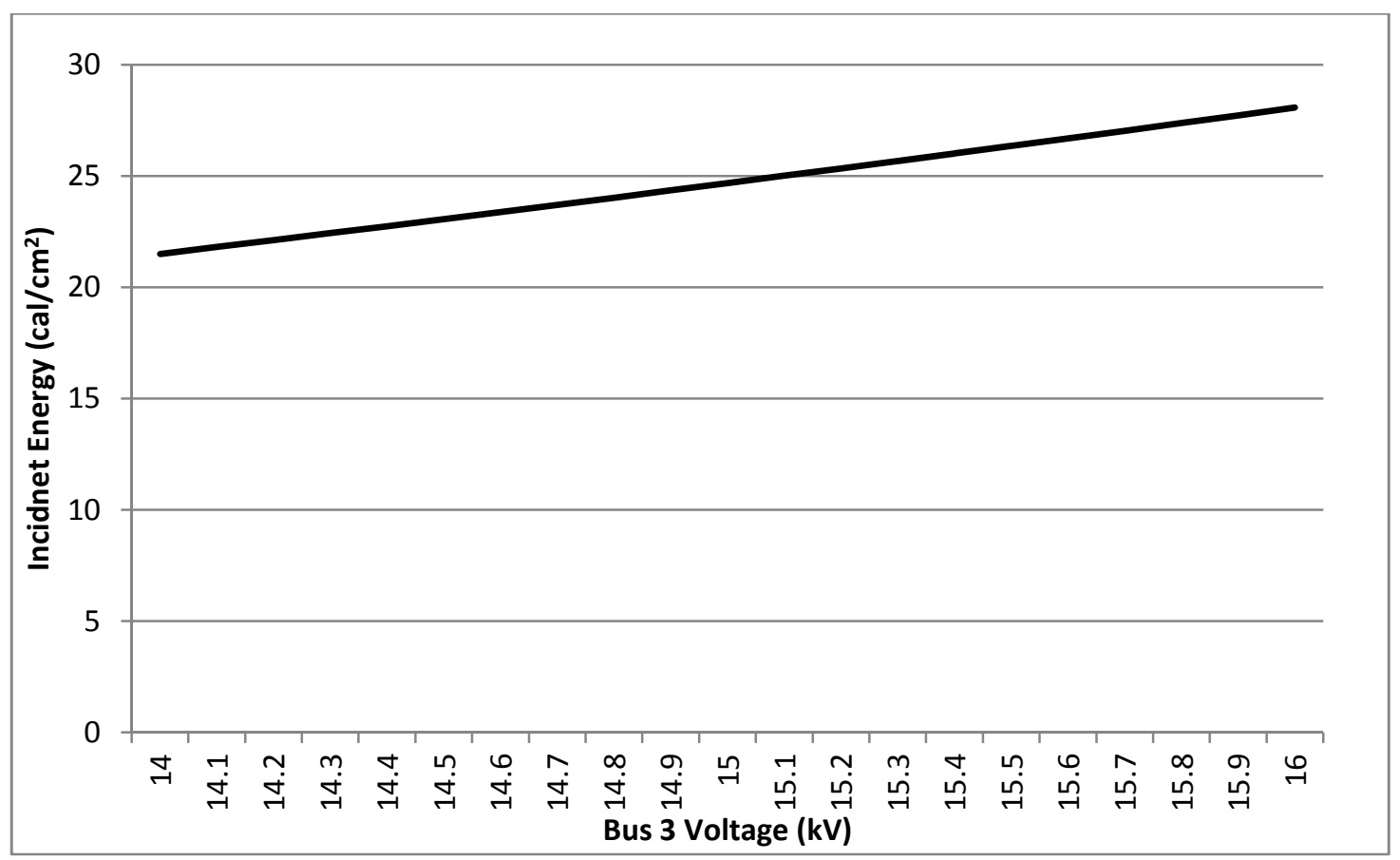

Figure 4.7: Incident energy based on Lee equation only

Figure 4.8 is a plot of the incident energy using the equation required based on the bus voltage. As it shows, at the $15 \mathrm{kV}$ voltage level, the incident energy makes a significant jump from around $1 \mathrm{cal} / \mathrm{cm}^{2}$ up to above $24 \mathrm{cal} / \mathrm{cm}^{2}$. This is due to the change to using the Lee Equation. It should be noted that the significant change does not occur in a real life application. The equipment under analysis would provide similar incident energy levels whether the voltage was at 14.9 or $15.1 \mathrm{kV}$. Using the higher incident energy could cause employees to wear over bearing PPE potentially causing more risk during their job duties and require wrong field labels placed on the equipment. Also, if the incident energy levels were high enough that the equipment would need to be de-energized before the employee could perform their job, this could cause jeopardy to the reliability of the system and lost revenue to the company. If lab tests were to be conducted on equipment with voltages greater than $15 \mathrm{kV}$, the derived equations potentially would continue linearly and not provide the significant jump causing unrealistic incident energy levels. Equipment around the $15 \mathrm{kV}$ level should be examined closely by hand to verify the correct incident energy is being applied to the system. 


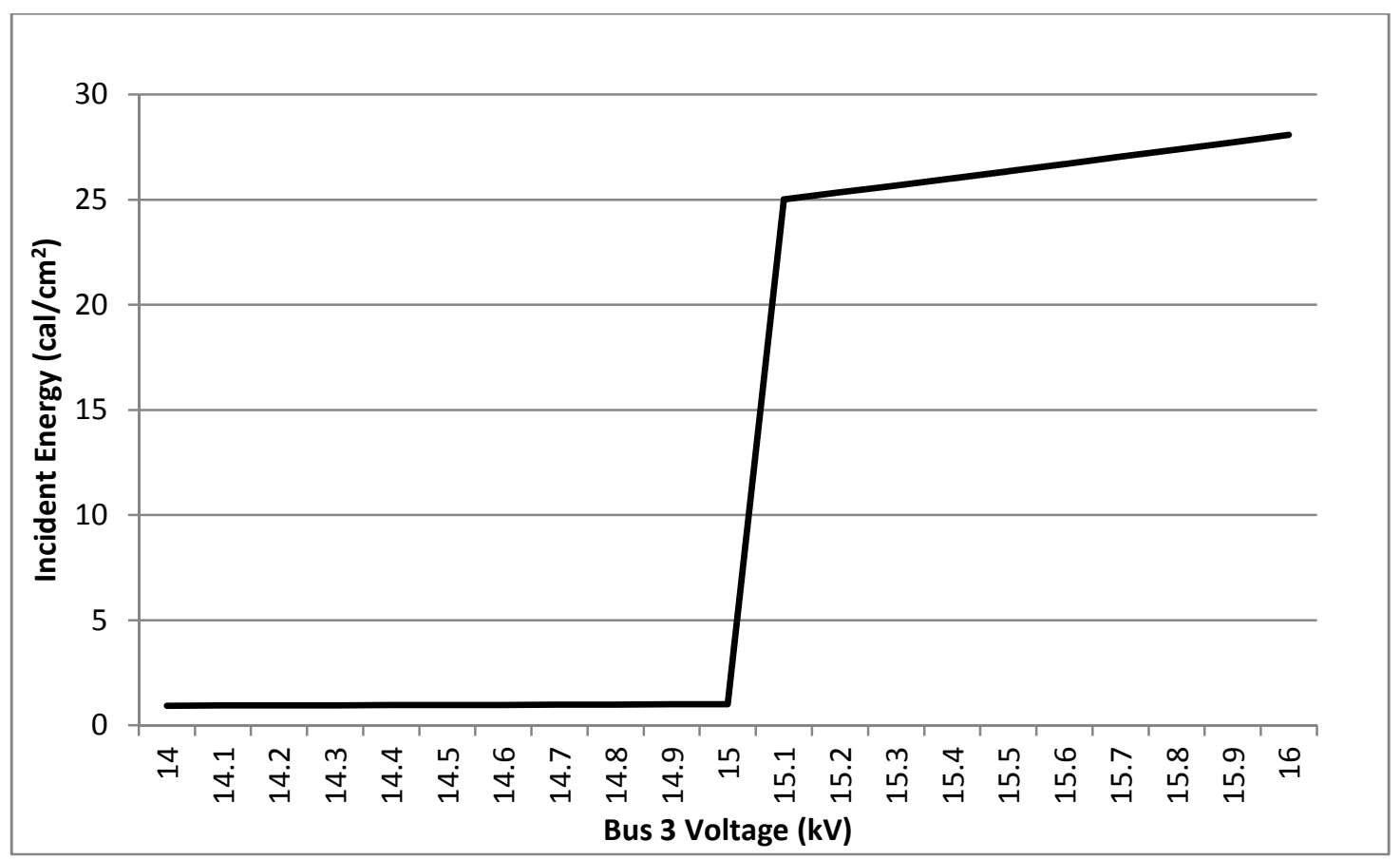

Figure 4.8: Incident energy following given bus voltage

Table 4.1 has the incident energy calculated from the voltage test system based on which set of equations were selected. These values were used to create Figures 4.3, 4.4 and 4.5 .

Table 4.2 has the incident energy calculated from the voltage test system based on which set of equations were selected. These values were used to create Figures 4.6, 4.7 and 4.8 . 
Table 4.1: $1 \mathrm{kV}$ voltage level test system incident energy values

\begin{tabular}{|c|c|c|c|}
\hline \multirow{2}{*}{$\begin{array}{c}\text { Bus Voltage } \\
(\mathrm{kV})\end{array}$} & \multicolumn{3}{|c|}{ Incident Energy $\left(\mathrm{cal} / \mathrm{cm}^{2}\right)$} \\
\cline { 2 - 4 } & Empirical Equation & Lee Equation & $\begin{array}{c}\text { Based on Bus } \\
\text { Voltage }\end{array}$ \\
\hline 0.5 & 109.89 & 1311.72 & 109.89 \\
\hline 0.6 & 173.60 & 1112.42 & 173.60 \\
\hline 0.7 & 274.65 & 966.29 & 274.65 \\
\hline 0.8 & 433.71 & 852.72 & 433.71 \\
\hline 0.9 & 683.28 & 761.94 & 683.28 \\
\hline 1 & 1074.61 & 688.33 & 688.33 \\
\hline 1.1 & 1688.36 & 628.00 & 628.00 \\
\hline 1.2 & 2651.74 & 578.13 & 578.13 \\
\hline 1.3 & 4167.63 & 536.78 & 536.78 \\
\hline 1.4 & 6558.29 & 502.31 & 502.31 \\
\hline 1.5 & 10345.38 & 473.69 & 473.69 \\
\hline
\end{tabular}

Table 4.2: $15 \mathrm{kV}$ voltage level test system incident energy values

\begin{tabular}{|c|c|c|c|}
\hline \multirow[b]{2}{*}{$\begin{array}{c}\text { Bus Voltage } \\
(\mathrm{kV})\end{array}$} & \multicolumn{3}{|c|}{ Incident Energy $\left(\mathrm{cal} / \mathrm{cm}^{2}\right)$} \\
\hline & Empirical Equation & Lee Equation & $\begin{array}{c}\text { Based on Bus } \\
\text { Voltage }\end{array}$ \\
\hline 14.0 & 0.94 & 21.50 & 0.94 \\
\hline 14.1 & 0.94 & 21.81 & 0.94 \\
\hline 14.2 & 0.95 & 22.12 & 0.95 \\
\hline 14.3 & 0.96 & 22.43 & 0.96 \\
\hline 14.4 & 0.96 & 22.75 & 0.96 \\
\hline 14.5 & 0.97 & 23.07 & 0.97 \\
\hline 14.6 & 0.98 & 23.38 & 0.98 \\
\hline 14.7 & 0.99 & 23.70 & 0.99 \\
\hline 14.8 & 0.99 & 24.03 & 0.99 \\
\hline 14.9 & 1.00 & 24.36 & 1.00 \\
\hline 15.0 & 1.01 & 24.68 & 1.01 \\
\hline 15.1 & 1.01 & 25.01 & 25.01 \\
\hline 15.2 & 1.02 & 25.34 & 25.34 \\
\hline 15.3 & 1.03 & 25.68 & 25.68 \\
\hline 15.4 & 1.04 & 26.02 & 26.02 \\
\hline 15.5 & 1.04 & 26.35 & 26.35 \\
\hline 15.6 & 1.05 & 26.69 & 26.69 \\
\hline 15.7 & 1.06 & 27.04 & 27.04 \\
\hline 15.8 & 1.06 & 27.38 & 27.38 \\
\hline 15.9 & 1.07 & 27.73 & 27.73 \\
\hline 16.0 & 1.08 & 28.08 & 28.08 \\
\hline
\end{tabular}

This is especially concerning due to the fact the NESC does not specify at what conditions the analysis is to be conducted; i.e. under light load or peak load conditions. 
Special consideration needs to be taken when analyzing systems where the voltage of the equipment can vary between two different sets of equations.

\subsection{Linearity of Incident Energy}

When comparing the incident energy levels over a range of possible current levels, both load and fault levels, it needs to be mentioned that the incident energy increase linearly and not exponentially. Figure 4.9 shows a standard U.S. U2 inverse time overcurrent curve of tripping times of a relay, either in a mechanical or electrical relay.

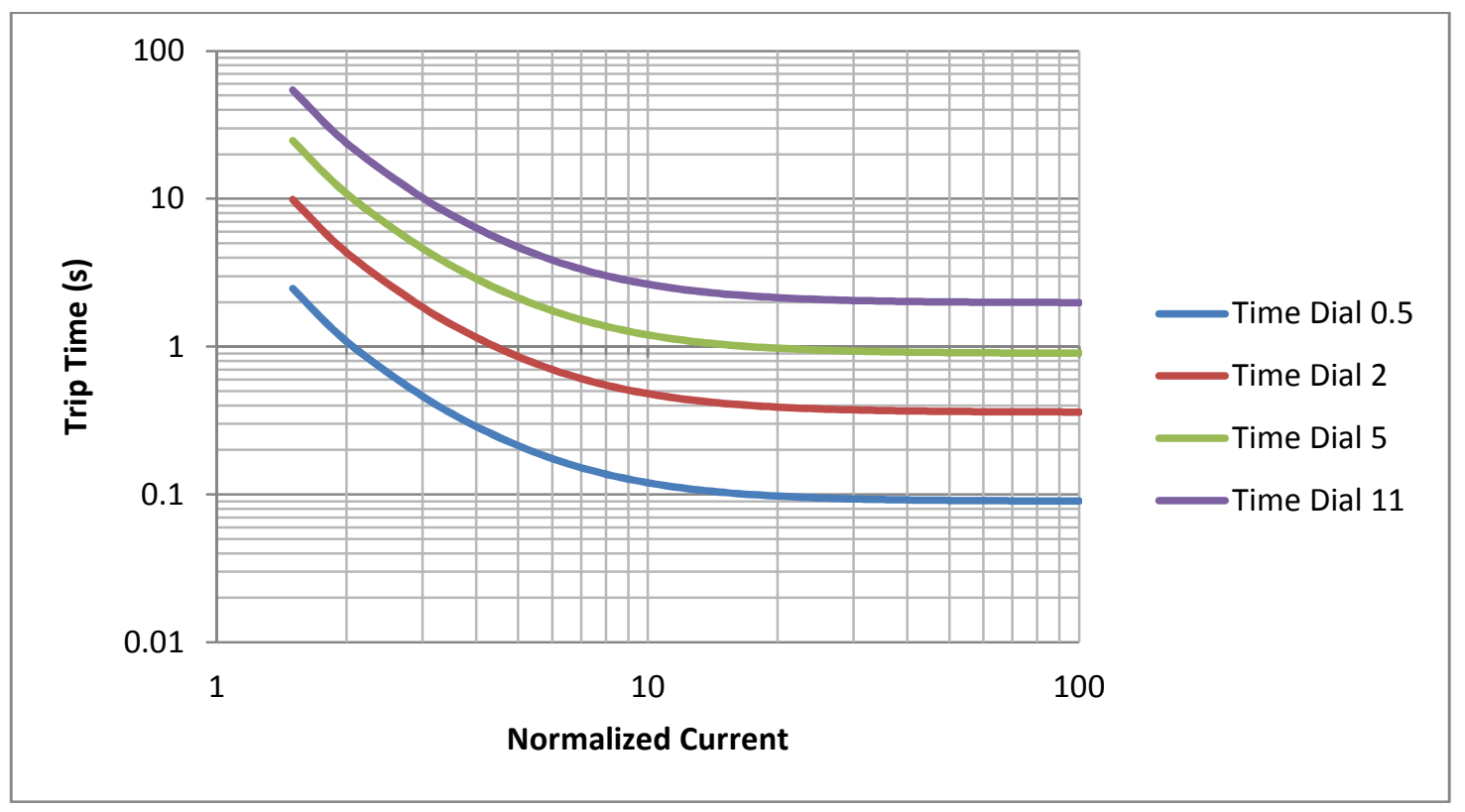

Figure 4.9: U.S. U2 inverse time overcurrent curves

When the overcurrent curves are applied to a piece of equipment and the energy through the equipment is calculated, it can be seen that the energy increases exponentially. Figure 4.10 depicts energy through a 6 inch piece of copper over a range of currents. In the equation for energy, the resistance is held constant; independent of the current. As the current increases the total time the current is applied to the equipment decreases due to the inverse overcurrent curve. Since the 
current is squared and the value of the current is much larger than any component in the equation, the energy curve becomes exponential.

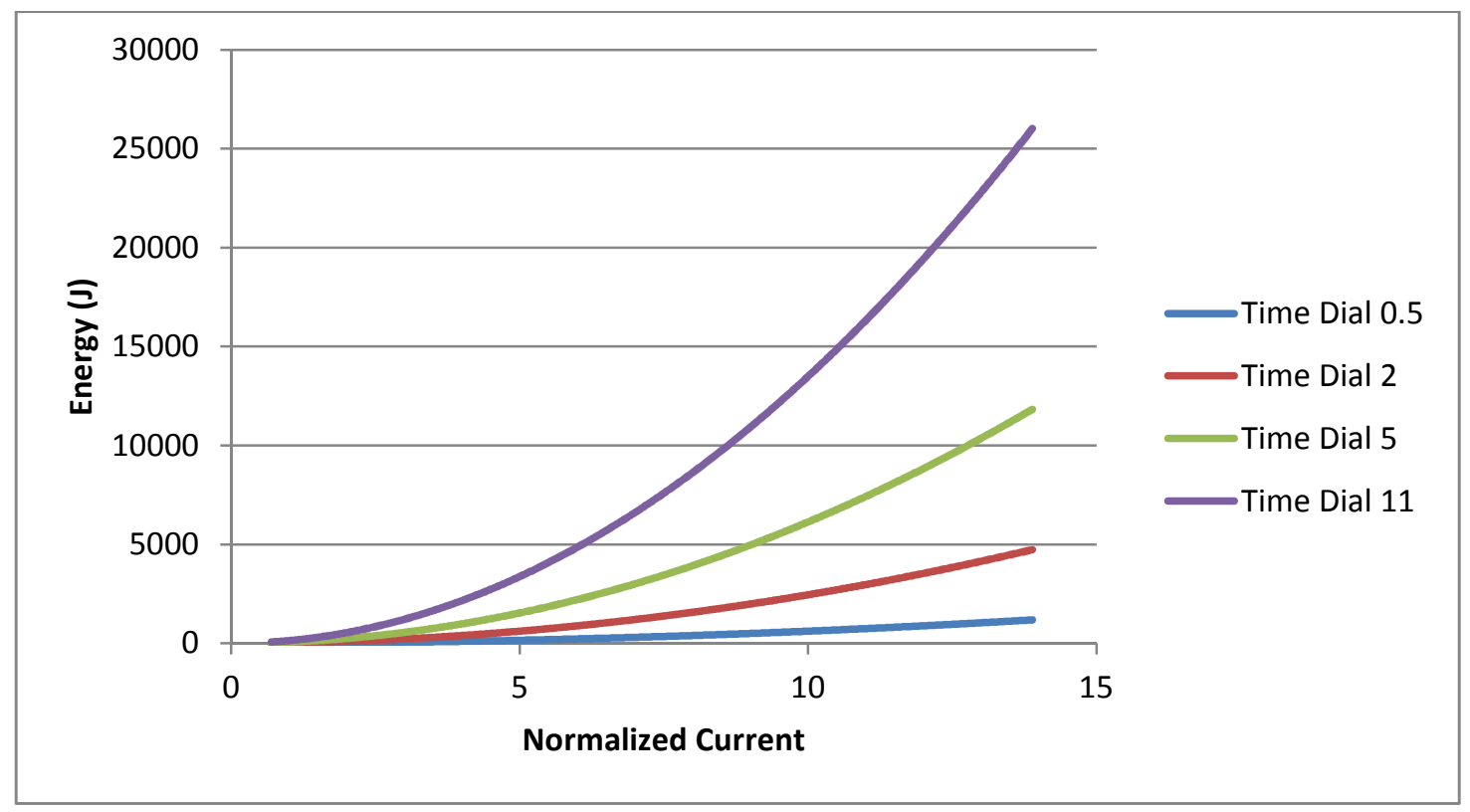

Figure 4.10: Energy through equipment as current increases

Now looking at the plots of the incident energy in Figure 4.11, using the same overcurrent curves and range of currents, the incident energy is linear. This is due to the fact that never in the equation is the current squared. 


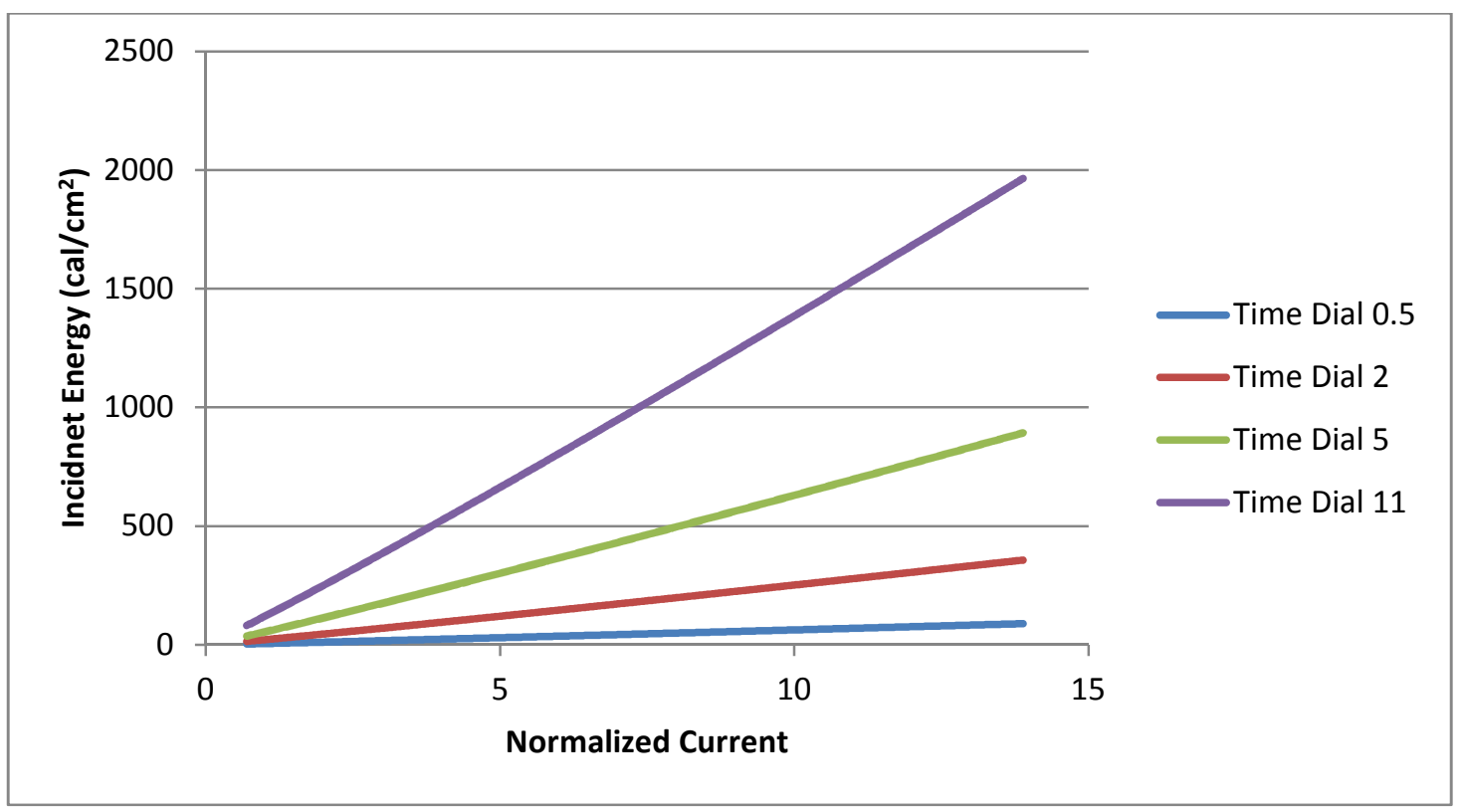

Figure 4.11: Potential incident energy in equipment as current increases

It needs to be noted that the energy calculated is only in Joules and is not directly comparable to cal $/ \mathrm{cm}^{2}$, which is what incident energy is measured in. These plots are shown to depict the discontinuity of the calculated level of energy between the two sets of equations. All data for the three plots in section 4.3 can be found in Appendix F. The parameters and assumptions used to build Figures 4.10 and 4.11 are as follows, the relay was using a 600/5 current transformer, tap setting of 6 amps, normal load current of 700 amps, a piece of wire 6 inches long with a diameter of 0.005 meters resulting in a resistance of $0.0001314 \mathrm{ohms}$ and a system voltage between $1 \mathrm{kV}$ and less than $15 \mathrm{kV}$.

\subsection{Radial Feeders}

Radial feeders are common on the distribution system in any utility power system. It's nearly impossible to network all load in the system due to a number of factors; location, cost and need to name a few. When determining the incident energy at the end of a radial feeder, the modeling can be simplified down to the source of the feeder and using the calculated incident energy for the whole feeder. 
If we refer to Figure 4.2 were we have two radial feeders connected to the system. In our case the load at bus 2 is 350 amps and bus 3 is 100 amps giving a total current at bus 1 to be 450 amps. Since bus 1 is the closest to the system it will have the highest fault current available with bus 2 being the second largest and bus 3 is the lowest on the feeder. When using inverse time overcurrent curves like those in Figure 4.6 and applying them to the fault currents on each bus down the feeder, we see that the largest incident energy will be at bus 1 and reduces as we move down the feeder towards bus 3 in the same fashion as Figure 4.8 depicts.

If a 50/51 relay was to be used instead of just a 51 relay, then the feeder would have two points where the incident energy would need to be calculated; one at the feeder source and one at the position were the 50 relay would stop operating and the 51 relay would start operating. 


\section{Chapter 5: Mitigation Methods and Hazard Reduction}

In order to conduct work on energized equipment, several different methods could be implemented to reduce the level of potential incident energy available. The ultimate hazard reduction would be to de-energize the equipment where the work is to take place. Unfortunately, working on energized equipment still needs to occur in the process of de-energizing the equipment requiring the maintenance. Below are different methods to be considered to reduce the arc-flash hazard.

\subsection{System Modifications}

One method to be evaluated when looking to reduce the incident energy level of an arc flash would be to remove system equipment from service. Removing a line from service, perhaps a parallel line to the location where work is to be conducted could reduce the fault current being supplied to the location. This would in turn reduce the arcing current. A study should be carried out though because lowering the fault current could increase the time needed to initiate the relay trip of the breaker.

Another piece of equipment to be considered is generators. If maintenance can be postponed to coordinate with a scheduled generator outage, this could also reduce fault current on the system. Again special consideration needs to be taken during these system configurations since a lower fault current can increase relay pickup times.

\subsection{Relay Application Changes}

There are a couple of changes that can be applied to relays before work is conducted to help reduce the level of arc-flash hazard presented to the personnel. The two following sections address each. 


\subsubsection{Application}

As proven earlier in section 4.2.1, applying the instantaneous trip application to a piece of equipment can reduce the potential incident energy provided to an employee. With the move from electromechanical relays to microprocessor based relays, applying this technique can be very simple. In many cases the change can be applied by a protection engineer in the home office by remote access. This ensures that the correct relay is changed and the correct settings are applied.

\subsubsection{Optical Detectors}

In addition to the conditions of an arc flash described above in section 2.1, light is also a product of the event. Light intensity can be thousands of times higher than normal ambient light. Optical sensors, in association with current transformers, can be used to detect an arc-flash condition and reduce tripping times down to 25 milliseconds. Both a fault current and sudden increase in light simultaneously will initiate a trip signal. This type of protection can be a stand-alone application. Two positives of using these relays are one, they requiring no coordination with existing protective devices and two, they are always in service and don't require any external intervention. This reduces the chance of forgetting to put a high speed protective device into service before the employee conducts the required work [7]. 


\section{Chapter 6: Conclusions and Recommendations}

This chapter provides observations and conclusions obtained during the research and implementation of this project. Suggestions and recommendations for future work are also presented.

\subsection{Observations and Conclusions}

- $\quad$ The arc-flash module provided by ASPEN® proved to perform as required based on the equations defined in IEEE Std. 1584-2002 except for the three instances where the code needed to be updated. The update ensured any other company/customer using the arc-flash module is using the correct code to perform their own analyses.

- Discontinuity around equipment at $15 \mathrm{kV}$ can be associated to two variables. The first is the system voltage is a variable in the Lee Equation and not in the empirical equation. This adds a multiple of at least 15.1 to the incident energy equation. The second is the full fault current is applied to the Lee Equation and the arcing current, which is a fraction of the fault current, is applied to the empirical equation.

- $\quad$ Two offline arc-flash analysis tools were developed to perform an analysis by hand without causing the user to enter values into multiple equations with the risk of entering the incorrect constants.

- The incident energy increases linearly in both the derived equations and in the Lee equation as the voltage is changed. When the voltage changes from 15 to $15.1 \mathrm{kV}$, the calculated incident energy can increase by 25 times the level with the Lee equation.

- The results of Minnesota Power's transmission system did not produce any current conditions where employees were at risk of an arc flash requiring PPE greater than employees currently wear. 


\subsection{Recommendations}

- $\quad$ Perform additional lab testing on equipment greater than $15 \mathrm{kV}$ to verify the continuity of the IEEE Std. 1584-2002 equations previously developed. This would provide data based equations for higher voltage distribution equipment.

- $\quad$ Perform an industry survey to find out how current standards are being applied; are industry participants using the IEEE Std. 1584-2002 equations or applying the tables provided in the NESC 2009 code. Also in the survey find out how often the industry is conducting an arc-flash analysis ensuring they are using current potential incident energy levels.

- $\quad$ Perform testing on the effects of the X/R ratio of the system and the DC offset of faults. X/R ratios and the DC offset can affect the fault magnitude which can affect the level of potential incident energy. Modeling of arcs in an EMTP program would show the effects.

The thermal effects of arcs have been studied extensively. Arcs also produce high levels of pressure which can cause injury to the employee. Perform detailed studies into the level of pressures associated with arcs and develop pressure PPE to be worn together with the thermal PPE to assist in preventing injury to the employee.

- Verify that $10 \mathrm{kV} /$ in was actually used as the dielectric strength of air in calculating the air gap in Table 410-2 of the NESC 2009 code.

- $\quad$ Perform lab testing on equipment, such as a $345 \mathrm{kV}$ breaker, were the more realistic fault to occur is a single line to ground. A bolted three phase fault on this equipment is unrealistic and could require the employee to wear unnecessary PPE which may hinder work activities.

- $\quad$ Perform a system analysis using a single line to ground fault, on equipment starting at given level such as $69 \mathrm{kV}$, where this type of fault is the most common especially given the needed conditions to create a bolted three phase fault.

- $\quad$ Verify the ASPEN® arc-flash module is updated with any changes made to assumptions or equations in IEEE Std. 1584-2002. 
- $\quad$ Perform a full system analysis if the equations in IEEE Std. 1584-2002 were to be updated based on the above mentioned lab testing.

- $\quad$ Use the results found in ASPEN® to assign arc-flash hazard levels rather than using NESC look-up tables. The tables provided by the NESC contradict the assumptions made in IEEE Std. 1584-2002 and could potentially mask a high arcflash level and put employees in situations that could cause them undue harm, even death.

- $\quad$ Rather than applying Exception 2 from the NESC 2009 code which allows employers to not perform an analysis on equipment below $1 \mathrm{kV}$, perform a full arc-flash hazard analysis. Not performing the analysis could put employees in situations that could cause them undue harm, even death. NESC 2012 now requires a detailed analysis or Tables 410-1, 410-2 or 410-3 must be used on equipment at or above 50 volts.

- $\quad$ Ensure an up-to-date protection database and model is maintained to reduce the analysis time if future studies are required.

- $\quad$ Develop a method to analyze multiple, pre-defined points on a power flow model by initializing a single run. This would greatly reduce the total run time and allow more time to analyze the output results. 


\section{References}

[1] J. C. Pfeiffer (2004). National Electrical Code Internet Connection [Online]. Available: http://www.mikeholt.com/mojonewsarchive/NECHTML/HTML/What-is-Arc-Flash 20040512.php

[2] Standard for Electrical Safety in the Workplace, NFPA 70E, 2004.

[3] National Electrical Safety Code, C2-2007, 2006.

[4] Graphic Products. (2009). Arc Flash Information Resource Center [Online]. Available: http://www.arcflash.me

[5] C. Inshaw and R. Wilson, "Arc Flash Hazard Analysis and Mitigation" in [58 Annual Conference for Protective Relay Engineers], [2005] @ [IEEE]. doi: [10.1109/CPRE.2005.1430429]

[6] R. Lee, “The Other Electrical Hazard: Electric Arc Blast Burns,” IEEE Trans. Ind. Appl., vol. IA-18, no. 3, pp. 246 - 251, May 1982.

[7] R. Wilson et al, "Tripping with the Speed of Light: Arc Flash Protection" in [60 ${ }^{\text {th }}$ Annual Conference for Protective Relay Engineers], [2007] @ [IEEE]. doi: [10.1109/CPRE.2007.359903]

[8] IEEE Guide for Arc-Flash Hazard Calculations, IEEE Std. 1584-2002, 2002.

[9] J. Phillips, "How to Perform an Arc-Flash Study in 12 Steps Part 1 of 3," NEC Digest Mag., pp. 29 - 34, Aug. 2007.

[10] J. Phillips, "How to Perform an Arc-Flash Study in 12 Steps Part 2 of 3," NEC Digest Mag., pp. 50 - 52, Oct. 2007.

[11] J. Phillips, "How to Perform an Arc-Flash Study in 12 Steps Part 3 of 3," NEC Digest Mag., pp. 38 - 42, Dec. 2007.

[12] R. Lee, "Pressures Developed by Arcs,". IEEE Trans.Ind. Appl., vol IA-23, no. 4, pp. 760 - 763, July/Aug. 1987.

[13] H.W. Tinsley et al, "Beyond the Calculations: Life After Arc Flash Analysis" in [Conference Record of Annual Pulp and Paper Industry Technical Conference], [2007] ( ) [IEEE]. doi: [10.1109/PAPCON.2007.4286307] 


\section{Appendix A Procedure for Arc-Flash Analysis}

The 12 steps below are to be used when conducting an arc-flash analysis.

1. Obtain data about the electrical system to be studied. This includes, but is not limited to, source/utility company, impedance data, overcurrent device data and arc-flash study parameters.

2. Develop a single line diagram to document and organize the arc-flash results.

3. Model the power system in an available computer program to assist in performing the required calculations based on the models developed in IEEE Std. 1584-2002.

4. Calculate the arcing short circuit. This is determined based on the available three phase bolted short circuit of the system and applying the IEEE Std. 1584-2002 equations.

5. Determine the clearing time of the overcurrent device upstream, using the time current characteristics, based on the arcing current.

6. Calculate the incident energy using the equations provided in IEEE Std. 1584-2002 based on the values found in steps four and five.

7. Calculate the flash protection boundary using the IEEE Std. 1584-2002 equations based on the incident energy found in step six.

8. Determine the minimum PPE required based on the incident energy found in step six.

9. Develop arc-flash warning labels including the incident energy, flash protection boundary, required PPE and shock hazard approach limits. 
10. Develop a report of the analysis including recommendations on how to reduce incident energy exposure.

11. Integrate the study results into the company's electrical safety program.

12. Train all affected workers on how to interpret the information provided by the study [9]-[11]. 


\section{Appendix B Arc-Flash Analyzer Verification Tools}

\section{B.1 Microsoft Excel Verification Tool}

Table B.1: Arc-flash hazard calculator

\begin{tabular}{|c|c|c|}
\hline \multicolumn{3}{|l|}{ Arc-Flash Hazard Calculator } \\
\hline \multicolumn{3}{|l|}{ User Inputs are in yellow } \\
\hline \multicolumn{3}{|l|}{ For system voltages $<1 \mathrm{kV}$, enter values below } \\
\hline Fault Current & & $\mathrm{kA}$ \\
\hline System Voltage & & $\mathrm{V}$ \\
\hline \multicolumn{3}{|l|}{$\mathrm{K}(-0.153$ for open air, -0.097 for enclosed $)$} \\
\hline Conductor Gap & & $\mathrm{mm}$ \\
\hline \multicolumn{3}{|l|}{$\mathrm{k}_{1}(-0.792$ for open air, -0.555 for enclosed $)$} \\
\hline \multicolumn{3}{|l|}{$\mathrm{k}_{2}(0$ for ungrounded, -0.113 for grounded $)$} \\
\hline Arc Duration & & $\sec$ \\
\hline Working Distance & & inches \\
\hline \multicolumn{3}{|l|}{$\begin{array}{l}\text { Distance Factor ( } 2 \text { for open air and cable, } 1.473 \text { for switch gear, } 1.641 \\
\text { for MCC and panels) }\end{array}$} \\
\hline $\begin{array}{lr} & \text { Arc Current } \\
\end{array}$ & \#NUM! & $\mathrm{kA}$ \\
\hline Normalized Incident Energy & \#NUM! & $\mathrm{J} / \mathrm{cm}^{2}$ \\
\hline Incident Energy & \#NUM! & $\mathrm{cal} / \mathrm{cm}^{2}$ \\
\hline \multicolumn{3}{|l|}{ For system voltages $>1 \mathrm{kV}$ to $15 \mathrm{kV}$, enter values below } \\
\hline Fault Current & & $\mathrm{kA}$ \\
\hline \multicolumn{3}{|l|}{$\mathrm{K}(-0.153$ for open air, -0.097 for enclosed $)$} \\
\hline Conductor Gap & & $\mathrm{mm}$ \\
\hline \multicolumn{3}{|l|}{$\mathrm{k}_{1}(-0.792$ for open air, -0.555 for enclosed $)$} \\
\hline \multicolumn{3}{|l|}{$\mathrm{k}_{2}(0$ for ungrounded, -0.113 for grounded $)$} \\
\hline Arc Duration & & sec \\
\hline Working Distance & & inches \\
\hline \multicolumn{3}{|l|}{ Distance Factor ( 2 for open air and cable, 0.973 for switch gear) } \\
\hline $\begin{array}{lr} & \text { Arc Current } \\
\end{array}$ & \#NUM! & $\mathrm{kA}$ \\
\hline Normalized Incident Energy & \#NUM! & $\mathrm{J} / \mathrm{cm}^{2}$ \\
\hline Incident Energy & \#NUM! & $\mathrm{cal} / \mathrm{cm}^{2}$ \\
\hline \multicolumn{3}{|l|}{ For system voltages $>15 \mathrm{kV}$, enter values below } \\
\hline System Voltage & & $\mathrm{kV}$ \\
\hline Fault Current & & $\mathrm{kA}$ \\
\hline Arc Duration & & $\sec$ \\
\hline Working Distance & & inches \\
\hline Incident Energy & \#DIV/0! & $\mathrm{cal} / \mathrm{cm}^{2}$ \\
\hline
\end{tabular}




\section{B.2 Microsoft Excel Verification Tool with Equations}

Table B.2: Arc-flash hazard calculator with equations

\begin{tabular}{|c|c|c|}
\hline \multicolumn{3}{|c|}{ Arc-Flash Hazard Calculator } \\
\hline User Inputs are in yellow & & \\
\hline \multicolumn{3}{|l|}{$\begin{array}{l}\text { For system voltages }<1 \mathrm{kV} \text {, enter values } \\
\text { below }\end{array}$} \\
\hline Fault Current & & $\mathrm{kA}$ \\
\hline System Voltage & & $\mathrm{V}$ \\
\hline \multicolumn{3}{|l|}{$\mathrm{K}(-0.153$ for open air, -0.097 for enclosed $)$} \\
\hline Conductor Gap & & $\mathrm{mm}$ \\
\hline \multicolumn{3}{|l|}{$\mathrm{k}_{1}(-0.792$ for open air, -0.555 for enclosed) } \\
\hline \multicolumn{3}{|l|}{$\mathrm{k}_{2}$ ( 0 for ungrounded, -0.113 for grounded $)$} \\
\hline Arc Duration & & sec \\
\hline Working Distance & & inches \\
\hline \multicolumn{3}{|l|}{$\begin{array}{l}\text { Distance Factor ( } 2 \text { for open air and cable, } \\
1.473 \text { for switch gear, } 1.641 \text { for MCC and } \\
\text { panels) }\end{array}$} \\
\hline Arc Current & $\begin{array}{l}10^{\wedge}(\mathrm{B} 8+(0.662 * \mathrm{LOG}(\mathrm{B} 6))+(0.0966 *( \\
\mathrm{B} 7 / 1000))+(0.000526 * \mathrm{~B} 9)+(0.5588 *( \\
\mathrm{B} 7 / 1000) * \mathrm{LOG}(\mathrm{B} 6))- \\
(0.00304 * \mathrm{~B} 9 * \mathrm{LOG}(\mathrm{B} 6)))\end{array}$ & $\mathrm{kA}$ \\
\hline Normalized Incident Energy & $\begin{array}{l}10^{\wedge}(\mathrm{B} 10+\mathrm{B} 11+(1.081 * \mathrm{LOG}(\mathrm{B} 15))+(0 \\
\left.\left..0011^{*} \mathrm{~B} 9\right)\right)\end{array}$ & $\mathrm{J} / \mathrm{cm}^{2}$ \\
\hline Incident Energy & $\begin{array}{l}4.184 * 1.5^{*} \mathrm{~B} 16 *(\mathrm{~B} 12 / 0.2)^{*}\left(\left(610^{\wedge} \mathrm{B} 14\right.\right. \\
\left.) /\left((25.4 * \mathrm{~B} 13)^{\wedge} \mathrm{B} 14\right)\right) * 0.24\end{array}$ & $\mathrm{cal} / \mathrm{cm}^{2}$ \\
\hline \multicolumn{3}{|l|}{$\begin{array}{l}\text { For system voltages }>1 \mathrm{kV} \text { to } 15 \mathrm{kV} \text {, enter } \\
\text { values below }\end{array}$} \\
\hline Fault Current & & $\mathrm{kA}$ \\
\hline \multicolumn{3}{|l|}{$\mathrm{K}(-0.153$ for open air, -0.097 for enclosed) } \\
\hline Conductor Gap & & $\mathrm{mm}$ \\
\hline \multicolumn{3}{|l|}{$\mathrm{k}_{1}(-0.792$ for open air, -0.555 for enclosed $)$} \\
\hline \multicolumn{3}{|l|}{$\mathrm{k}_{2}$ ( 0 for ungrounded, -0.113 for grounded $)$} \\
\hline Arc Duration & & $\mathrm{sec}$ \\
\hline Working Distance & & inches \\
\hline \multicolumn{3}{|l|}{$\begin{array}{l}\text { Distance Factor ( } 2 \text { for open air and cable, } \\
0.973 \text { for switch gear) }\end{array}$} \\
\hline Arc Current & $10^{\wedge}(0.00402+(0.983 * \operatorname{LOG}(\mathrm{B} 21)))$ & $\mathrm{kA}$ \\
\hline Normalized Incident Energy & $\begin{array}{l}10^{\wedge}(\mathrm{B} 24+\mathrm{B} 25+(1.081 * \mathrm{LOG}(\mathrm{B} 29))+(0 \\
\left.\left..0011^{*} \mathrm{~B} 23\right)\right)\end{array}$ & $\mathrm{J} / \mathrm{cm}^{2}$ \\
\hline Incident Energy & $\begin{array}{l}4.184 * \mathrm{~B} 30^{*}(\mathrm{~B} 26 / 0.2)^{*}\left(\left(610^{\wedge} \mathrm{B} 28\right) /((2\right. \\
\left.\left.5.4 * \mathrm{~B} 27)^{\wedge} \mathrm{B} 28\right)\right)^{*} 0.24\end{array}$ & $\mathrm{cal} / \mathrm{cm}^{2}$ \\
\hline For system voltages $>15 \mathrm{kV}$, enter values & & \\
\hline
\end{tabular}




\begin{tabular}{|l|l|l|}
\hline below & & \\
\hline & & \\
\hline System Voltage & & $\mathrm{kV}$ \\
\hline Fault Current & & $\mathrm{kA}$ \\
\hline Arc Duration & & $\mathrm{sec}$ \\
\hline Working Distance & & inches \\
\hline & $\left(2.142^{*} 10^{\wedge} 6^{*} \mathrm{~B} 35^{*} \mathrm{~B} 36^{*}\left(\mathrm{~B} 37 /\left(\left(25.4^{*}\right.\right.\right.\right.$ & \\
& $\left.\left.\mathrm{B} 38)^{\wedge} 2\right)\right)^{*} 0.24$ & $\mathrm{cal} / \mathrm{cm}^{2}$ \\
\hline
\end{tabular}

\section{B.3 MATLAB Verification Tool}

\section{FlashCalcs}

Enter the Values Below to Calculate Incident Energy

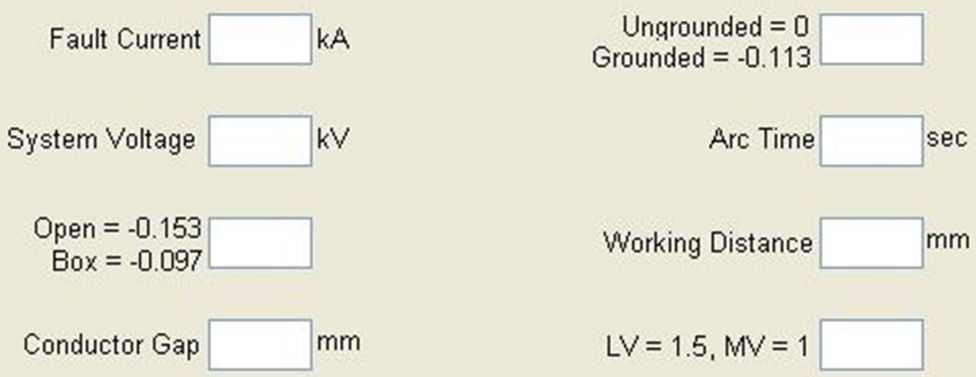

LV \& MV Open Air and Cable $=2$, MV Switchgear $=0.973$

LV Switchgear $=1.473$, LV MCC \& Panels $=1.641$

\section{Calculate}

Arc Current

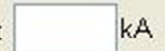

Incident Energy

$\mathrm{cal} / \mathrm{cm}^{\wedge} 2$

Protection Boundary $\mathrm{ft}$

Figure B.1: MATLAB verification tool user interface

\section{B.3.1 MATLAB Program}

function varargout $=$ FlashCalcs (varargin)

$\%$ FLASHCALCS M-file for FlashCalcs.fig

$\%$ FLASHCALCS, by itself, creates a new FLASHCALCS or raises the existing $\%$ singleton*.

$\%$

$\% \quad \mathrm{H}=$ FLASHCALCS returns the handle to a new FLASHCALCS or the handle to $\%$ the existing singleton*.

$\%$ 


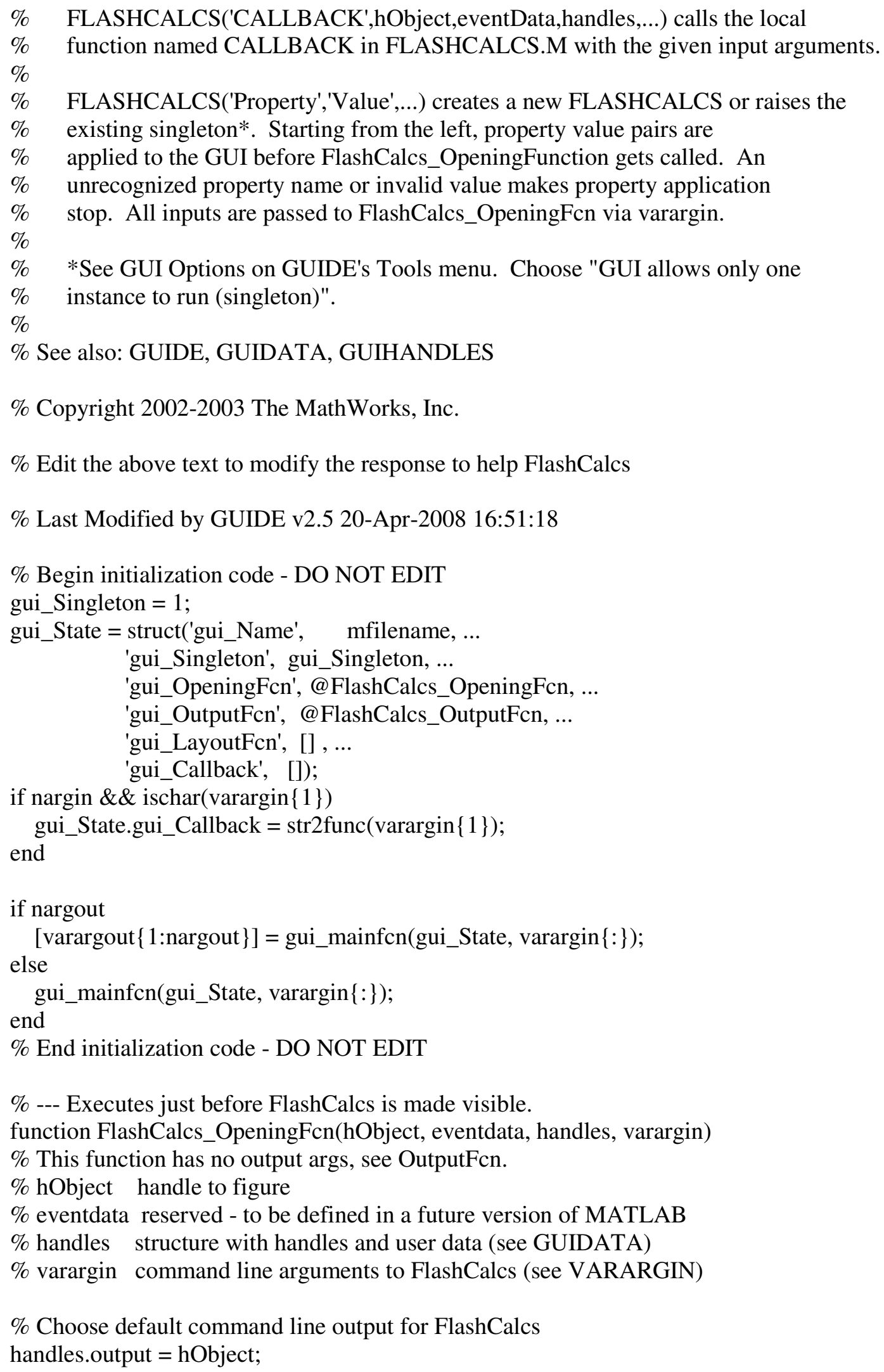


$\%$ Update handles structure

guidata(hObject, handles);

$\%$ UIWAIT makes FlashCalcs wait for user response (see UIRESUME)

$\%$ uiwait(handles.figure1);

$\%$--- Outputs from this function are returned to the command line.

function varargout $=$ FlashCalcs_OutputFen(hObject, eventdata, handles)

$\%$ varargout cell array for returning output args (see VARARGOUT);

$\%$ hObject handle to figure

$\%$ eventdata reserved - to be defined in a future version of MATLAB

$\%$ handles structure with handles and user data (see GUIDATA)

$\%$ Get default command line output from handles structure

varargout $\{1\}=$ handles.output;

function edit1_Callback(hObject, eventdata, handles)

$\%$ hObject handle to edit1 (see GCBO)

$\%$ eventdata reserved - to be defined in a future version of MATLAB

$\%$ handles structure with handles and user data (see GUIDATA)

$\%$ Hints: get(hObject,'String') returns contents of edit1 as text

$\% \quad$ str2double(get(hObject,'String')) returns contents of edit1 as a double

$\%$--- Executes during object creation, after setting all properties.

function edit1_CreateFcn(hObject, eventdata, handles)

$\%$ hObject handle to edit1 (see GCBO)

$\%$ eventdata reserved - to be defined in a future version of MATLAB

$\%$ handles empty - handles not created until after all CreateFcns called

\% Hint: edit controls usually have a white background on Windows.

$\% \quad$ See ISPC and COMPUTER.

if ispc

set(hObject,'BackgroundColor','white');

else

set(hObject,'BackgroundColor',get( 0 ,'defaultUicontrolBackgroundColor')); end

function edit2_Callback(hObject, eventdata, handles)

$\%$ hObject handle to edit2 (see GCBO)

$\%$ eventdata reserved - to be defined in a future version of MATLAB

$\%$ handles structure with handles and user data (see GUIDATA)

\% Hints: get(hObject,'String') returns contents of edit2 as text

$\% \quad$ str2double(get(hObject,'String')) returns contents of edit2 as a double

$\%$--- Executes during object creation, after setting all properties.

function edit2_CreateFcn(hObject, eventdata, handles) 
$\%$ hObject handle to edit2 (see GCBO)

$\%$ eventdata reserved - to be defined in a future version of MATLAB

$\%$ handles empty - handles not created until after all CreateFcns called

$\%$ Hint: edit controls usually have a white background on Windows.

$\% \quad$ See ISPC and COMPUTER.

if ispc

set(hObject,'BackgroundColor','white');

else

set(hObject,'BackgroundColor',get( 0 ,'defaultUicontrolBackgroundColor')); end

function edit3_Callback(hObject, eventdata, handles)

$\%$ hObject handle to edit3 (see GCBO)

$\%$ eventdata reserved - to be defined in a future version of MATLAB

$\%$ handles structure with handles and user data (see GUIDATA)

$\%$ Hints: get(hObject,'String') returns contents of edit3 as text

$\% \quad$ str2double(get(hObject,'String')) returns contents of edit3 as a double

$\%$--- Executes during object creation, after setting all properties.

function edit3_CreateFcn(hObject, eventdata, handles)

$\%$ hObject handle to edit3 (see GCBO)

$\%$ eventdata reserved - to be defined in a future version of MATLAB

$\%$ handles empty - handles not created until after all CreateFcns called

\% Hint: edit controls usually have a white background on Windows.

$\% \quad$ See ISPC and COMPUTER.

if ispc

set(hObject,'BackgroundColor','white');

else

set(hObject,'BackgroundColor', get( 0 ,'defaultUicontrolBackgroundColor')); end

function edit4_Callback(hObject, eventdata, handles)

$\%$ hObject handle to edit4 (see GCBO)

$\%$ eventdata reserved - to be defined in a future version of MATLAB

$\%$ handles structure with handles and user data (see GUIDATA)

$\%$ Hints: get(hObject,'String') returns contents of edit 4 as text

$\% \quad$ str2double(get(hObject,'String')) returns contents of edit4 as a double

$\%$--- Executes during object creation, after setting all properties.

function edit4_CreateFcn(hObject, eventdata, handles)

$\%$ hObject handle to edit4 (see GCBO)

$\%$ eventdata reserved - to be defined in a future version of MATLAB

$\%$ handles empty - handles not created until after all CreateFcns called

$\%$ Hint: edit controls usually have a white background on Windows. 


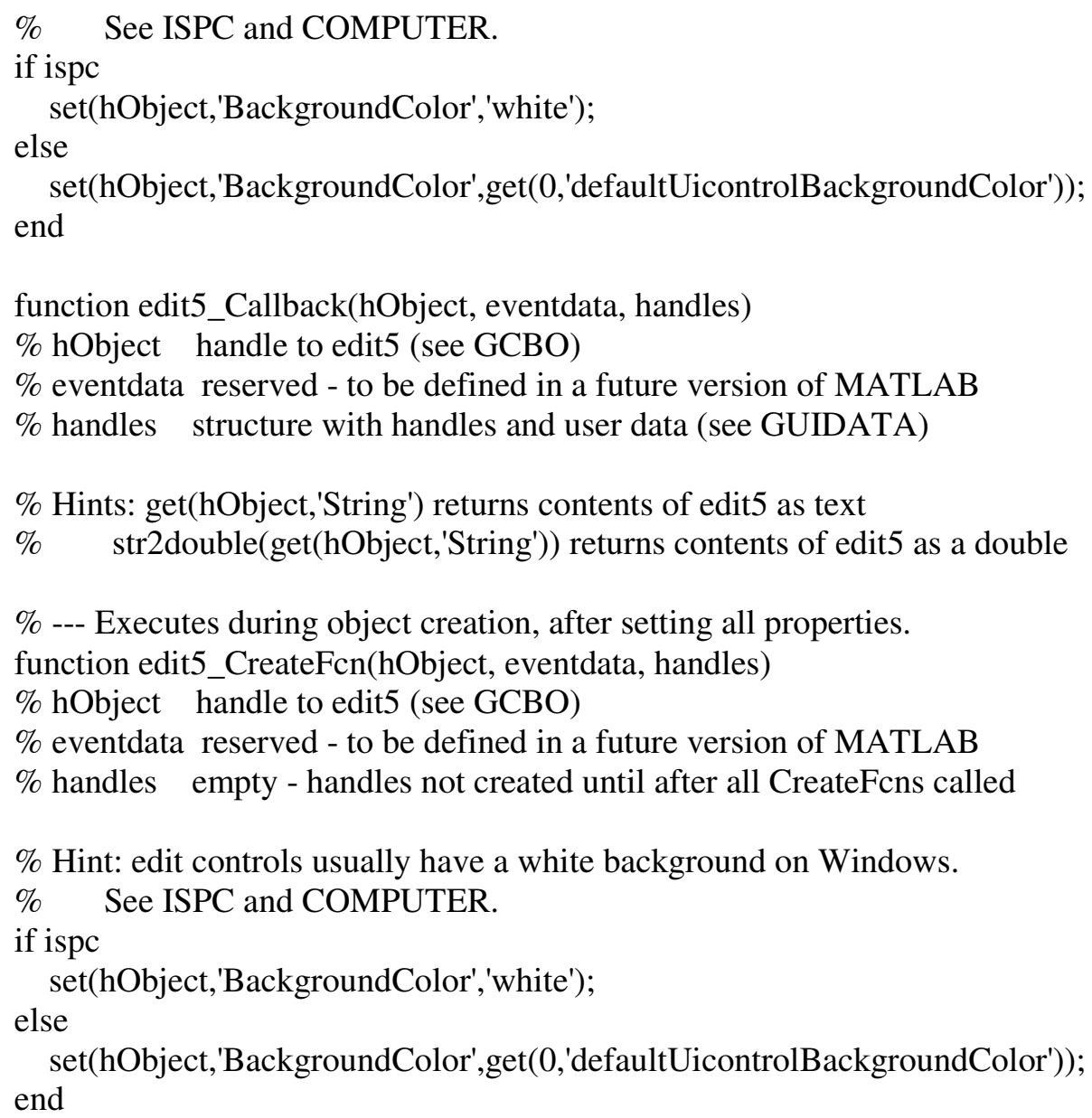




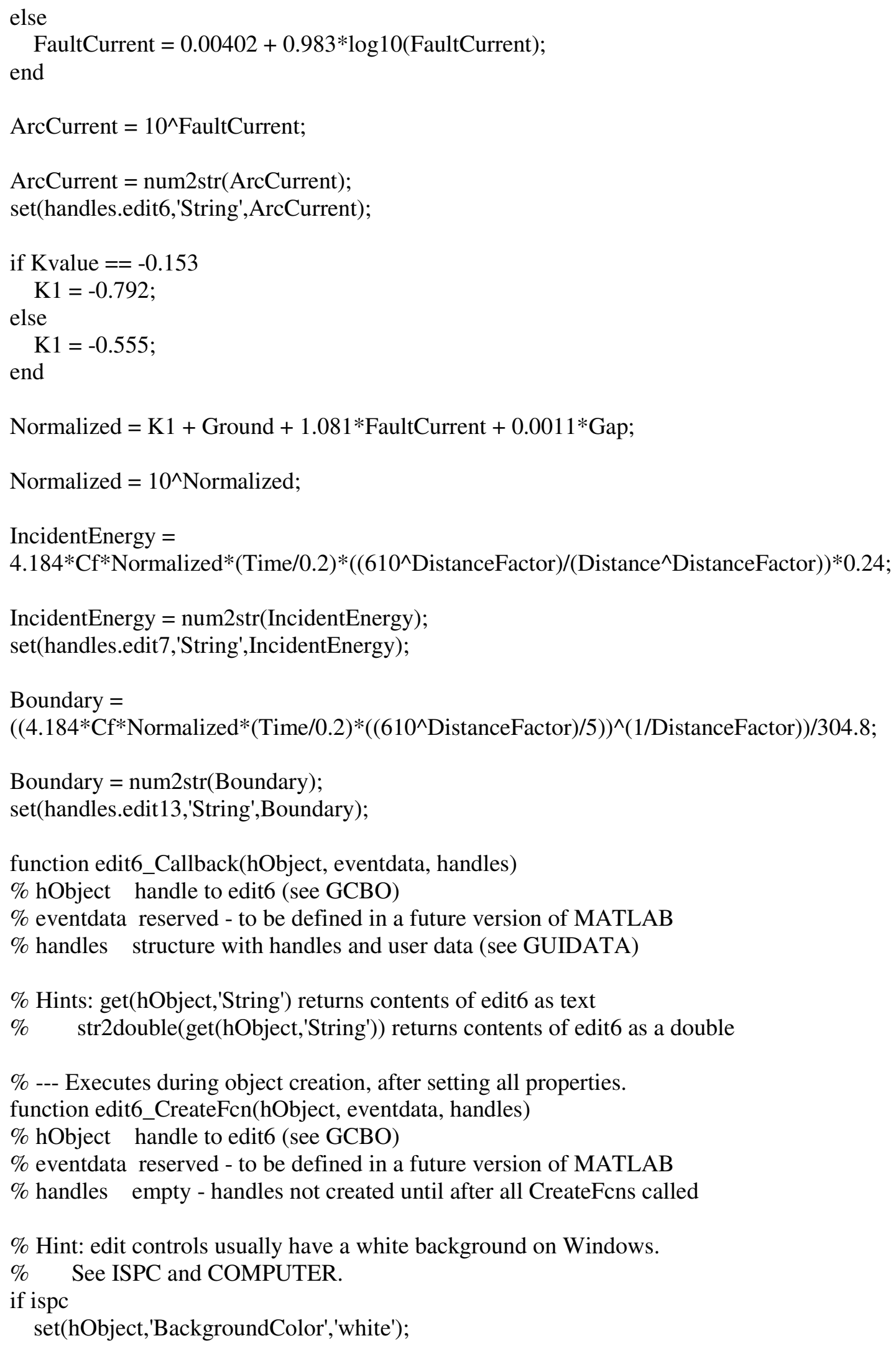




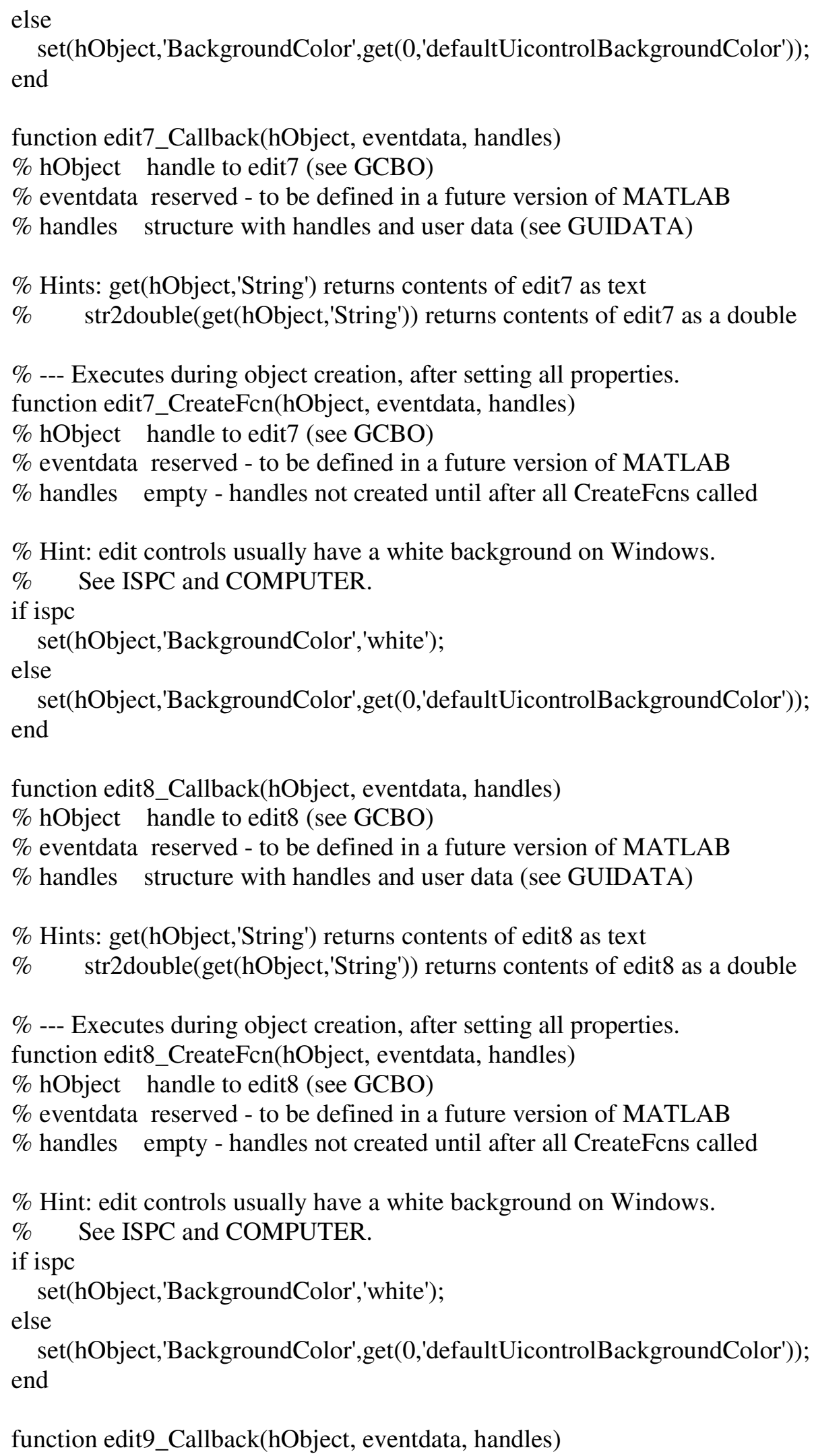


$\%$ hObject handle to edit9 (see GCBO)

$\%$ eventdata reserved - to be defined in a future version of MATLAB

$\%$ handles structure with handles and user data (see GUIDATA)

$\%$ Hints: get(hObject,'String') returns contents of edit9 as text

$\% \quad$ str2double(get(hObject,'String')) returns contents of edit9 as a double

$\%$--- Executes during object creation, after setting all properties.

function edit9_CreateFcn(hObject, eventdata, handles)

$\%$ hObject handle to edit9 (see GCBO)

$\%$ eventdata reserved - to be defined in a future version of MATLAB

$\%$ handles empty - handles not created until after all CreateFcns called

\% Hint: edit controls usually have a white background on Windows.

$\% \quad$ See ISPC and COMPUTER.

if ispc

set(hObject,'BackgroundColor','white');

else

set(hObject,'BackgroundColor',get( 0 ,'defaultUicontrolBackgroundColor'));

end

function edit10_Callback(hObject, eventdata, handles)

$\%$ hObject handle to edit8 (see GCBO)

$\%$ eventdata reserved - to be defined in a future version of MATLAB

$\%$ handles structure with handles and user data (see GUIDATA)

$\%$ Hints: get(hObject,'String') returns contents of edit8 as text

$\% \quad$ str2double(get(hObject,'String')) returns contents of edit8 as a double

$\%$--- Executes during object creation, after setting all properties.

function edit10_CreateFcn(hObject, eventdata, handles)

$\%$ hObject handle to edit8 (see GCBO)

$\%$ eventdata reserved - to be defined in a future version of MATLAB

$\%$ handles empty - handles not created until after all CreateFcns called

$\%$ Hint: edit controls usually have a white background on Windows.

$\% \quad$ See ISPC and COMPUTER.

if ispc

set(hObject,'BackgroundColor','white');

else

set(hObject,'BackgroundColor',get( 0 ,'defaultUicontrolBackgroundColor')); end

function edit11_Callback(hObject, eventdata, handles)

$\%$ hObject handle to edit10 (see GCBO)

$\%$ eventdata reserved - to be defined in a future version of MATLAB

$\%$ handles structure with handles and user data (see GUIDATA)

\% Hints: get(hObject,'String') returns contents of edit10 as text 
$\%$--- Executes during object creation, after setting all properties.

function edit11_CreateFcn(hObject, eventdata, handles)

$\%$ hObject handle to edit10 (see GCBO)

$\%$ eventdata reserved - to be defined in a future version of MATLAB

$\%$ handles empty - handles not created until after all CreateFcns called

$\%$ Hint: edit controls usually have a white background on Windows.

$\% \quad$ See ISPC and COMPUTER.

if ispc

set(hObject,'BackgroundColor','white');

else

set(hObject,'BackgroundColor',get( 0 ,'defaultUicontrolBackgroundColor'));

end

function edit12_Callback(hObject, eventdata, handles)

$\%$ hObject handle to edit9 (see GCBO)

$\%$ eventdata reserved - to be defined in a future version of MATLAB

$\%$ handles structure with handles and user data (see GUIDATA)

$\%$ Hints: get(hObject,'String') returns contents of edit9 as text

$\% \quad$ str2double(get(hObject,'String')) returns contents of edit9 as a double

$\%$--- Executes during object creation, after setting all properties.

function edit12_CreateFcn(hObject, eventdata, handles)

$\%$ hObject handle to edit9 (see GCBO)

$\%$ eventdata reserved - to be defined in a future version of MATLAB

$\%$ handles empty - handles not created until after all CreateFcns called

$\%$ Hint: edit controls usually have a white background on Windows.

$\% \quad$ See ISPC and COMPUTER.

if ispc

set(hObject,'BackgroundColor','white');

else

set(hObject,'BackgroundColor',get( 0 ,'defaultUicontrolBackgroundColor')); end

function edit13_Callback(hObject, eventdata, handles)

$\%$ hObject handle to edit13 (see GCBO)

$\%$ eventdata reserved - to be defined in a future version of MATLAB

$\%$ handles structure with handles and user data (see GUIDATA)

$\%$ Hints: get(hObject,'String') returns contents of edit13 as text

$\% \quad$ str2double(get(hObject,'String')) returns contents of edit13 as a double

$\%$--- Executes during object creation, after setting all properties.

function edit13_CreateFcn(hObject, eventdata, handles)

$\%$ hObject handle to edit13 (see GCBO) 
$\%$ eventdata reserved - to be defined in a future version of MATLAB

$\%$ handles empty - handles not created until after all CreateFcns called

$\%$ Hint: edit controls usually have a white background on Windows.

$\% \quad$ See ISPC and COMPUTER.

if ispc

set(hObject,'BackgroundColor','white');

else

set(hObject,'BackgroundColor',get( 0 ,'defaultUicontrolBackgroundColor')); end 


\section{Appendix C Example System Data}

\section{C.1 System Online Diagram}

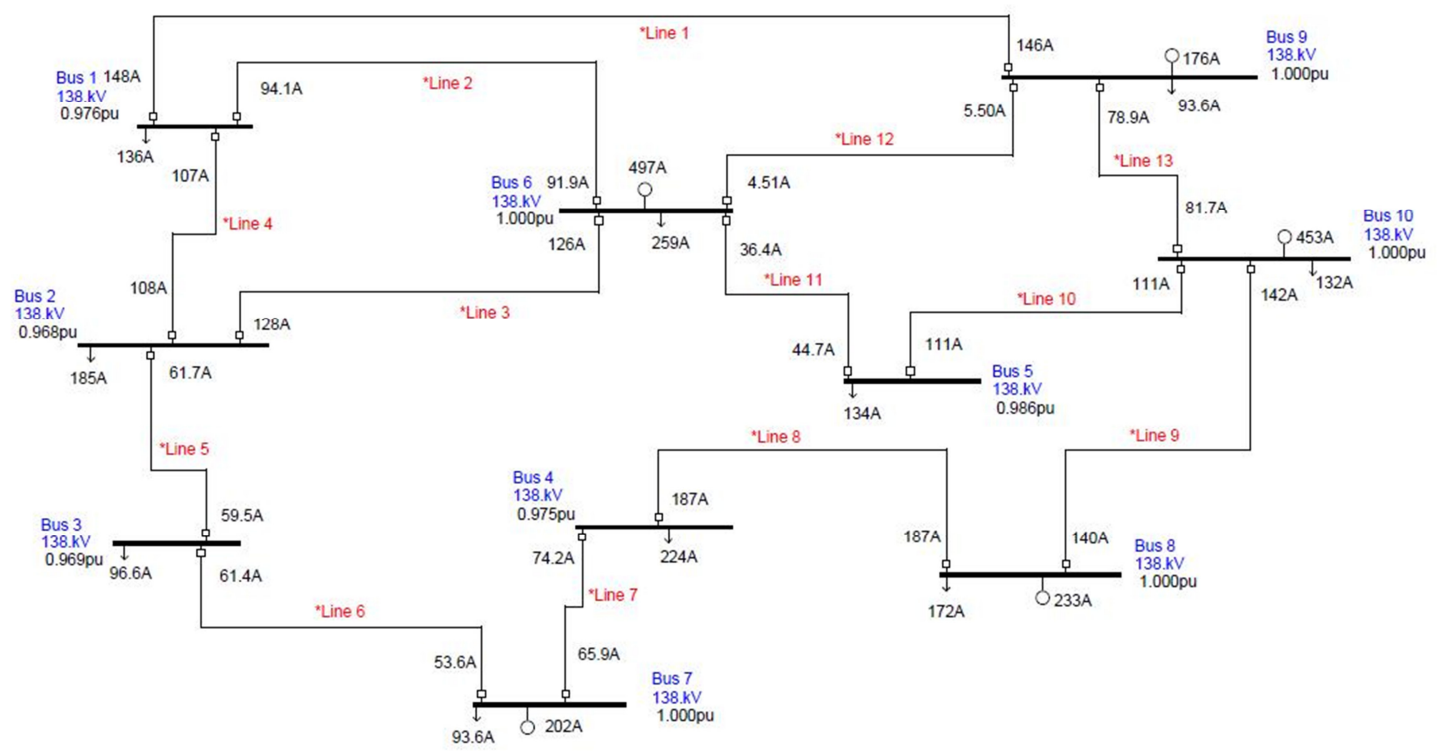

Figure C.1: Example system oneline diagram

\section{C.2 Generator Data}

Table C.1: Example system generator data

\begin{tabular}{|c|c|c|c|c|}
\hline \multirow[b]{2}{*}{ Bus } & \multirow{2}{*}{$\begin{array}{c}\text { Min/Max } \\
\text { MW }\end{array}$} & \multirow{2}{*}{$\begin{array}{c}\text { Min/Max } \\
\text { MVAR }\end{array}$} & \multicolumn{2}{|c|}{$\begin{array}{l}\text { Values in per unit all for Subtansient, } \\
\text { Transient, Synchronous, Negative } \\
\text { Sequence and Zero Sequence }\end{array}$} \\
\hline & & & $\mathbf{R}$ & $\mathbf{X}$ \\
\hline 6 & $25 / 150$ & $-10 / 50$ & 0.09149 & 0.09149 \\
\hline 7 & $10 / 75$ & $0 / 20$ & 0.09576 & 0.09576 \\
\hline 8 & $15 / 100$ & $-5 / 30$ & 0.09237 & 0.09237 \\
\hline 9 & $5 / 80$ & $-5 / 25$ & 0.0958 & 0.0958 \\
\hline 10 & $50 / 200$ & $-15 / 60$ & 0.09044 & 0.09044 \\
\hline
\end{tabular}




\section{C.3 Load Data}

Table C.2: Example system load data

\begin{tabular}{|c|c|c|}
\hline Bus & Pload (MW) & Qload (MVAR) \\
\hline 1 & 30 & 10 \\
\hline 2 & 40 & 15 \\
\hline 3 & 20 & 10 \\
\hline 4 & 50 & 15 \\
\hline 5 & 30 & 10 \\
\hline 6 & 60 & 15 \\
\hline 7 & 20 & 10 \\
\hline 8 & 40 & 10 \\
\hline 9 & 20 & 10 \\
\hline 10 & 30 & 10 \\
\hline
\end{tabular}

\section{C.4 Line Data}

Table C.3: Example system line data

\begin{tabular}{|c|c|c|c|c|c|c|c|c|}
\hline & \multicolumn{9}{|c|}{ Values in per unit } \\
\cline { 2 - 9 } Line & $\mathbf{R}$ & $\mathbf{X}$ & $\mathbf{B 1}$ & $\mathbf{B 2}$ & $\mathbf{R - 0}$ & $\mathbf{X - 0}$ & $\mathbf{B 1 - 0}$ & $\mathbf{B 2 - 0}$ \\
\hline 1 & 0.0366 & 0.14639 & 0.01093 & 0.01093 & 0.0366 & 0.014639 & 0.01093 & 0.01093 \\
\hline 2 & 0.0549 & 0.22873 & 0.01093 & 0.01093 & 0.0366 & 0.014639 & 0.01093 & 0.01093 \\
\hline 3 & 0.0549 & 0.22873 & 0.01093 & 0.01093 & 0.0366 & 0.014639 & 0.01093 & 0.01093 \\
\hline 4 & 0.0183 & 0.07319 & 0.00546 & 0.00546 & 0.0366 & 0.014639 & 0.01093 & 0.01093 \\
\hline 5 & 0.0549 & 0.22873 & 0.01093 & 0.01093 & 0.0366 & 0.014639 & 0.01093 & 0.01093 \\
\hline 6 & 0.0549 & 0.22873 & 0.01093 & 0.01093 & 0.0366 & 0.014639 & 0.01093 & 0.01093 \\
\hline 7 & 0.0366 & 0.14639 & 0.01093 & 0.01093 & 0.0366 & 0.014639 & 0.01093 & 0.01093 \\
\hline 8 & 0.0549 & 0.22873 & 0.01093 & 0.01093 & 0.0366 & 0.014639 & 0.01093 & 0.01093 \\
\hline 9 & 0.0366 & 0.14639 & 0.01093 & 0.01093 & 0.0366 & 0.014639 & 0.01093 & 0.01093 \\
\hline 10 & 0.0549 & 0.22873 & 0.01093 & 0.01093 & 0.0366 & 0.014639 & 0.01093 & 0.01093 \\
\hline 11 & 0.0366 & 0.14639 & 0.01093 & 0.01093 & 0.0366 & 0.014639 & 0.01093 & 0.01093 \\
\hline 12 & 0.0183 & 0.07319 & 0.00546 & 0.00546 & 0.0366 & 0.014639 & 0.01093 & 0.01093 \\
\hline 13 & 0.07319 & 0.29278 & 0.01366 & 0.01366 & 0.0366 & 0.014639 & 0.01093 & 0.01093 \\
\hline
\end{tabular}




\section{C.5 Relay Data}

Table C.4: Example system relay data

\begin{tabular}{|c|c|c|c|c|c|}
\hline Relay Name & CT Ratio & $\begin{array}{c}\text { Tap Setting } \\
\text { (Pickup) }\end{array}$ & $\begin{array}{c}\text { Time } \\
\text { Dial }\end{array}$ & $\begin{array}{c}\text { Relay } \\
\text { Curve }\end{array}$ & $\begin{array}{c}\text { Tap } \\
\text { Unit }\end{array}$ \\
\hline Line 1 Bus 1 & $120 / 5$ & 6.25 & 0.5 & ABB CO-9 & GC2 \\
\hline Line 2 Bus 1 & $120 / 5$ & 4 & 0.5 & ABB CO-9 & GC2 \\
\hline Line 4 Bus 1 & $120 / 5$ & 4.5 & 0.5 & ABB CO-9 & GC2 \\
\hline Line 1 Bus 9 & $120 / 5$ & 6.25 & 0.5 & ABB CO-9 & GC2 \\
\hline Line 12 Bus 9 & $60 / 5$ & 0.5 & 0.5 & ABB CO-9 & GC1 \\
\hline Line 13 Bus 9 & $120 / 5$ & 3.5 & 0.5 & ABB CO-9 & GC2 \\
\hline Line 13 Bus 10 & $120 / 5$ & 3.5 & 0.5 & ABB CO-9 & GC2 \\
\hline Line 9 Bus 10 & $120 / 5$ & 6 & 0.5 & ABB CO-9 & GC2 \\
\hline Line 10 Bus 10 & $120 / 5$ & 4.75 & 0.5 & ABB CO-9 & GC2 \\
\hline Line 9 Bus 8 & $120 / 5$ & 6 & 0.5 & ABB CO-9 & GC2 \\
\hline Line 8 Bus 8 & $120 / 5$ & 8 & 0.5 & ABB CO-9 & GC3 \\
\hline Line 8 Bus 4 & $120 / 5$ & 8 & 0.5 & ABB CO-9 & GC3 \\
\hline Line 7 Bus 7 & $120 / 5$ & 3 & 0.5 & ABB CO-9 & GC1 \\
\hline Line 6 Bus 7 & $120 / 5$ & 2.25 & 0.5 & ABB CO-9 & GC1 \\
\hline Line 6 Bus 3 & $120 / 5$ & 2.75 & 0.5 & ABB CO-9 & GC1 \\
\hline Line 5 Bus 3 & $120 / 5$ & 2.5 & 0.5 & ABB CO-9 & GC1 \\
\hline Line 5 Bus 2 & $120 / 5$ & 2.75 & 0.5 & ABB CO-9 & GC1 \\
\hline Line 3 Bus 2 & $120 / 5$ & 5.5 & 0.5 & ABB CO-9 & GC2 \\
\hline Line 4 Bus 2 & $120 / 5$ & 4.75 & 0.5 & ABB CO-9 & GC2 \\
\hline Line 3 Bus 6 & $120 / 5$ & 5.5 & 0.5 & ABB CO-9 & GC2 \\
\hline Line 2 Bus 6 & $120 / 5$ & 4 & 0.5 & ABB CO-9 & GC2 \\
\hline Line 12 Bus 6 & $60 / 5$ & 0.5 & 0.5 & ABB CO-9 & GC1 \\
\hline Line 11 Bus 6 & $120 / 5$ & 1.75 & 0.5 & ABB CO-9 & GC1 \\
\hline Line 11 Bus 5 & $120 / 5$ & 2 & 0.5 & ABB CO-9 & GC1 \\
\hline Line 10 Bus 5 & $120 / 5$ & 4.75 & 0.5 & ABB CO-9 & GC2 \\
\hline Line 7 Bus 4 & $120 / 5$ & 3.25 & 0.5 & ABB CO-9 & GC1 \\
\hline
\end{tabular}




\section{Appendix D Example System ASPEN@ Output Data}

\section{D.1 ASPEN® Results}

Bus $=$ Bus $1138 \mathrm{kV}$

Equipment category $=$ Open Air

Grounded $=$ Yes

Enclosed $=$ No

Breaker interrupting time $($ cycles $)=3.0$

Working distance (inches) $=53.0$

Conductor gap $(\mathrm{mm})=202.4$

Bolted 3PH fault current $(\mathrm{kA})=4.228$

Arcing current $(\mathrm{kA})=4.228$

Interrupting device $=[\mathrm{OC}$ relay $]$ Line 4 Bus 1 on $\quad 0$ Bus $1 \quad 138 . \mathrm{kV}-\quad 0$ Bus $2 \quad 138 . \mathrm{kV} 1 \mathrm{~L}$

Line 1

Clearing time (seconds) $=0.11$

Incident energy $(\mathrm{cal} / \mathrm{cm} 2)=17.70$

Interrupting device $@ 85 \%$ current $=[\mathrm{OC}$ relay $]$ Line 4 Bus 1 on $\quad 0$ Bus $1 \quad 138 . \mathrm{kV}-\quad 0$ Bus 2

138.kV 1 L Line 1

Clearing time @85\% current (seconds)=0.11

Incident energy@85\% current $(\mathrm{cal} / \mathrm{cm} 2)=15.79$

Required PPE cat. per NFPA 70E $=3$

PPE cat. 1 flash hazard boundary (inches) $=203.5$

PPE cat. 2 flash hazard boundary (inches) $=99.7$

PPE cat. 3 flash hazard boundary (inches) $=78.8$

PPE cat. 4 flash hazard boundary (inches) $=44.6$

Bus $=$ Bus $2138 \mathrm{kV}$

Equipment category $=$ Open Air

Grounded $=$ Yes

Enclosed $=$ No

Breaker interrupting time (cycles) $=3.0$

Working distance $($ inches $)=53.0$

Conductor gap $(\mathrm{mm})=202.4$

Bolted 3PH fault current $(\mathrm{kA})=3.819$

Arcing current $(\mathrm{kA})=3.819$

Interrupting device $=\left[\begin{array}{lllll}\mathrm{OC} \text { relay }\end{array}\right]$ Line 12 Bus 6 on $\quad 0$ Bus $6 \quad 138 . \mathrm{kV}-\quad 0$ Bus $9 \quad 138 . \mathrm{kV} 1$

L Line 11

Clearing time (seconds) $=0.09$

Incident energy $(\mathrm{cal} / \mathrm{cm} 2)=13.43$

Interrupting device @ 85\% current = [OC relay $]$ Line 12 Bus 6 on $\quad 0$ Bus $6 \quad 138 . \mathrm{kV}-\quad 0$ Bus 9

138.kV 1 L Line 1

Clearing time@85\% current (seconds)=0.09

Incident energy @85\% current $(\mathrm{cal} / \mathrm{cm} 2)=11.48$

Required PPE cat. per NFPA 70E $=3$

PPE cat. 1 flash hazard boundary (inches) $=177.3$

PPE cat. 2 flash hazard boundary (inches) $=86.9$

PPE cat. 3 flash hazard boundary (inches) $=68.7$

PPE cat. 4 flash hazard boundary (inches) $=38.8$ 
Bus = Bus $3138 \mathrm{kV}$

Equipment category = Open Air

Grounded $=$ Yes

Enclosed $=$ No

Breaker interrupting time $($ cycles $)=3.0$

Working distance (inches) $=53.0$

Conductor gap $(\mathrm{mm})=202.4$

Bolted 3PH fault current $(\mathrm{kA})=2.471$

Arcing current $(\mathrm{kA})=2.471$

Interrupting device $=[\mathrm{OC}$ relay $]$ Line 6 Bus 7 on $\quad 0$ Bus $7 \quad 138 . \mathrm{kV}-\quad 0$ Bus $3 \quad 138 . \mathrm{kV} 2 \mathrm{~L}$

Line 6

Clearing time $($ seconds) $=0.09$

Incident energy $(\mathrm{cal} / \mathrm{cm} 2)=9.05$

Interrupting device @85\% current = [OC relay $]$ Line 6 Bus 7 on $\quad 0$ Bus $7 \quad 138 . \mathrm{kV}$ - $\quad 0$ Bus 3

138.kV 2 L Line 6

Clearing time@85\% current (seconds)=0.10

Incident energy @ 85\% current $(\mathrm{cal} / \mathrm{cm} 2)=7.87$

Required PPE cat. per NFPA 70E = 3

PPE cat. 1 flash hazard boundary (inches) $=145.6$

PPE cat. 2 flash hazard boundary (inches) $=71.3$

PPE cat. 3 flash hazard boundary (inches) $=56.4$

PPE cat. 4 flash hazard boundary (inches) $=31.9$

Bus $=$ Bus $4138 \mathrm{kV}$

Equipment category = Open Air

Grounded $=$ Yes

Enclosed $=$ No

Breaker interrupting time $($ cycles $)=3.0$

Working distance (inches) $=53.0$

Conductor gap $(\mathrm{mm})=202.4$

Bolted $3 \mathrm{PH}$ fault current $(\mathrm{kA})=3.150$

Arcing current $(\mathrm{kA})=3.150$

Interrupting device $=[\mathrm{OC}$ relay $]$ Line 7 Bus 7 on $\quad 0$ Bus $7 \quad 138 . \mathrm{kV}-\quad 0$ Bus $4 \quad 138 . \mathrm{kV} 1 \mathrm{~L}$

Line 7

Clearing time $($ seconds) $=0.09$

Incident energy $(\mathrm{cal} / \mathrm{cm} 2)=11.57$

Interrupting device $@ 85 \%$ current = [OC relay $]$ Line 7 Bus 7 on $\quad 0$ Bus $7 \quad 138 . \mathrm{kV}$ - $\quad 0$ Bus 4 138.kV 1 L Line 7

Clearing time @85\% current (seconds)=0.10

Incident energy @85\% current $(\mathrm{cal} / \mathrm{cm} 2)=10.06$

Required PPE cat. per NFPA 70E = 3

PPE cat. 1 flash hazard boundary (inches) $=164.5$

PPE cat. 2 flash hazard boundary (inches) $=80.6$

PPE cat. 3 flash hazard boundary (inches) $=63.7$

PPE cat. 4 flash hazard boundary (inches) $=36.1$

Bus $=$ Bus $5138 \mathrm{kV}$ 
Equipment category $=$ Open Air

Grounded $=$ Yes

Enclosed $=$ No

Breaker interrupting time (cycles) $=3.0$

Working distance (inches) $=53.0$

Conductor gap $(\mathrm{mm})=202.4$

Bolted 3PH fault current $(\mathrm{kA})=3.395$

Arcing current $(\mathrm{kA})=3.395$

Interrupting device $=\left[\begin{array}{lllll}\mathrm{OC} \text { relay }\end{array}\right]$ Line 12 Bus 6 on $\quad 0$ Bus $6 \quad 138 . \mathrm{kV}-\quad 0$ Bus $9 \quad 138 . \mathrm{kV} 1$

L Line 11

Clearing time (seconds) $=0.09$

Incident energy $(\mathrm{cal} / \mathrm{cm} 2)=11.91$

Interrupting device @ 85\% current = [OC relay $]$ Line 12 Bus 6 on $\quad 0$ Bus $6 \quad 138 . \mathrm{kV}-\quad 0$ Bus 9

138.kV 1 L Line 1

Clearing time @ 85\% current (seconds) = 0.09

Incident energy @85\% current $(\mathrm{cal} / \mathrm{cm} 2)=10.12$

Required PPE cat. per NFPA 70E $=3$

PPE cat. 1 flash hazard boundary (inches) $=167.0$

PPE cat. 2 flash hazard boundary (inches) $=81.8$

PPE cat. 3 flash hazard boundary (inches) $=64.7$

PPE cat. 4 flash hazard boundary (inches) $=36.6$

Bus $=$ Bus $6138 \mathrm{kV}$

Equipment category $=$ Open Air

Grounded $=$ Yes

Enclosed $=$ No

Breaker interrupting time $($ cycles $)=3.0$

Working distance (inches) $=53.0$

Conductor gap $(\mathrm{mm})=202.4$

Bolted 3PH fault current $(\mathrm{kA})=7.465$

Arcing current $(\mathrm{kA})=7.465$

Interrupting device $=\left[\begin{array}{lllll}\mathrm{OC} \text { relay }\end{array}\right]$ Line 12 Bus 6 on $\quad 0$ Bus $6 \quad 138 . \mathrm{kV}-\quad 0$ Bus $9 \quad 138 . \mathrm{kV} 1$

L Line 11

Clearing time (seconds) $=0.09$

Incident energy $(\mathrm{cal} / \mathrm{cm} 2)=26.19$

Interrupting device @ $85 \%$ current $=[\mathrm{OC}$ relay $]$ Line 12 Bus 6 on $\quad 0$ Bus $6 \quad 138 . \mathrm{kV}-\quad 0$ Bus 9

138.kV 1 L Line 1

Clearing time @85\% current (seconds)=0.09

Incident energy @85\% current $(\mathrm{cal} / \mathrm{cm} 2)=22.26$

Required PPE cat. per NFPA 70E $=4$

PPE cat. 1 flash hazard boundary (inches) $=247.6$

PPE cat. 2 flash hazard boundary (inches) $=121.3$

PPE cat. 3 flash hazard boundary (inches) $=95.9$

PPE cat. 4 flash hazard boundary (inches) $=54.2$

Bus $=$ Bus $7138 \mathrm{kV}$

Equipment category $=$ Open Air

Grounded $=$ Yes

Enclosed $=$ No 
Breaker interrupting time (cycles) $=3.0$

Working distance (inches) $=53.0$

Conductor gap $(\mathrm{mm})=202.4$

Bolted 3PH fault current $(\mathrm{kA})=4.892$

Arcing current $(\mathrm{kA})=4.892$

Interrupting device $=\left[\begin{array}{lllll}\mathrm{OC} \text { relay }\end{array}\right]$ Line 7 Bus 7 on $\quad 0$ Bus $7 \quad 138 . \mathrm{kV}-\quad 0$ Bus $4 \quad 138 . \mathrm{kV} 1 \mathrm{~L}$

Line 7

Clearing time $($ seconds $)=0.11$

Incident energy $(\mathrm{cal} / \mathrm{cm} 2)=20.04$

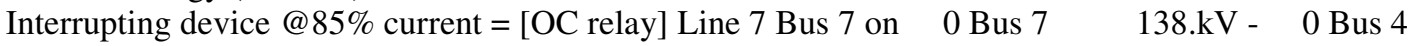

138.kV 1 L Line 7

Clearing time @ $85 \%$ current (seconds) $=0.11$

Incident energy @ 85\% current $(\mathrm{cal} / \mathrm{cm} 2)=17.86$

Required PPE cat. per NFPA 70E $=3$

PPE cat. 1 flash hazard boundary (inches) $=216.6$

PPE cat. 2 flash hazard boundary (inches) $=106.1$

PPE cat. 3 flash hazard boundary (inches) $=83.9$

PPE cat. 4 flash hazard boundary (inches) $=47.5$

Bus $=$ Bus $8138 \mathrm{kV}$

Equipment category $=$ Open Air

Grounded $=$ Yes

Enclosed $=$ No

Breaker interrupting time $($ cycles $)=3.0$

Working distance (inches) $=53.0$

Conductor gap $(\mathrm{mm})=202.4$

Bolted 3PH fault current $(\mathrm{kA})=5.986$

Arcing current $(\mathrm{kA})=5.986$

Interrupting device $=\left[\begin{array}{lllll}\mathrm{OC} \text { relay }\end{array}\right]$ Line 9 Bus 10 on $\quad 0$ Bus $10 \quad 138 . \mathrm{kV}-\quad 0$ Bus $8 \quad 138 . \mathrm{kV} 2$

L Line 12

Clearing time $($ seconds $)=0.11$

Incident energy $(\mathrm{cal} / \mathrm{cm} 2)=24.84$

Interrupting device $@ 85 \%$ current $=[\mathrm{OC}$ relay $]$ Line 9 Bus 10 on $\quad 0$ Bus $10 \quad 138 . \mathrm{kV}-\quad 0$ Bus 8

138.kV 2 L Line 1

Clearing time @ 85\% current (seconds) $=0.11$

Incident energy @85\% current $(\mathrm{cal} / \mathrm{cm} 2)=22.16$

Required PPE cat. per NFPA 70E $=3$

PPE cat. 1 flash hazard boundary (inches) $=241.1$

PPE cat. 2 flash hazard boundary (inches) $=118.1$

PPE cat. 3 flash hazard boundary (inches) $=93.4$

PPE cat. 4 flash hazard boundary (inches) $=52.8$

Bus $=$ Bus $9138 \mathrm{kV}$

Equipment category $=$ Open Air

Grounded $=$ Yes

Enclosed $=$ No

Breaker interrupting time (cycles) $=3.0$

Working distance $($ inches $)=53.0$

Conductor gap $(\mathrm{mm})=202.4$ 
Bolted 3PH fault current $(\mathrm{kA})=7.380$

Arcing current $(\mathrm{kA})=7.380$

Interrupting device $=[\mathrm{OC}$ relay $]$ Line 12 Bus 9 on $\quad 0$ Bus $9 \quad 138 . \mathrm{kV}-\quad 0$ Bus $6 \quad 138 . \mathrm{kV} 1$

L Line 11

Clearing time $($ seconds $)=0.09$

Incident energy $(\mathrm{cal} / \mathrm{cm} 2)=25.89$

Interrupting device @ 85\% current = [OC relay $]$ Line 12 Bus 9 on $\quad 0$ Bus $9 \quad 138 . \mathrm{kV}$ - 0 Bus 6 138.kV 1 L Line 1

Clearing time @ 85\% current (seconds)=0.09

Incident energy @85\% current $(\mathrm{cal} / \mathrm{cm} 2)=22.01$

Required PPE cat. per NFPA 70E $=4$

PPE cat. 1 flash hazard boundary (inches) $=246.2$

PPE cat. 2 flash hazard boundary (inches) $=120.6$

PPE cat. 3 flash hazard boundary (inches) $=95.4$

PPE cat. 4 flash hazard boundary (inches) $=53.9$

Bus $=$ Bus $10138 \mathrm{kV}$

Equipment category = Open Air

Grounded $=$ Yes

Enclosed $=$ No

Breaker interrupting time (cycles) $=3.0$

Working distance (inches) $=53.0$

Conductor gap $(\mathrm{mm})=202.4$

Bolted 3PH fault current $(\mathrm{kA})=6.860$

Arcing current $(\mathrm{kA})=6.860$

Interrupting device $=[$ OC relay $]$ Line 12 Bus 9 on $\quad 0$ Bus $9 \quad 138 . \mathrm{kV}-\quad 0$ Bus $6 \quad 138 . \mathrm{kV} 1$

L Line 11

Clearing time (seconds) $=0.10$

Incident energy $(\mathrm{cal} / \mathrm{cm} 2)=26.41$

Interrupting device @85\% current = [OC relay $]$ Line 12 Bus 9 on $\quad 0$ Bus $9 \quad 138 . \mathrm{kV}$ - 0 Bus 6

138.kV 1 L Line 1

Clearing time@85\% current (seconds)=0.10

Incident energy @85\% current $(\mathrm{cal} / \mathrm{cm} 2)=23.27$

Required PPE cat. per NFPA 70E $=4$

PPE cat. 1 flash hazard boundary (inches) $=248.6$

PPE cat. 2 flash hazard boundary (inches) $=121.8$

PPE cat. 3 flash hazard boundary (inches) $=96.3$

PPE cat. 4 flash hazard boundary (inches) $=54.5$ 


\section{D.2 PPE Levels Required}

Table D.1: Example system PPE level requirements

\begin{tabular}{|c|c|c|c|c|c|c|c|}
\hline \multirow[b]{2}{*}{ 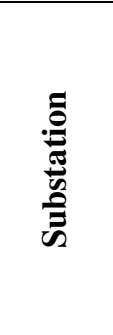 } & \multirow{2}{*}{ 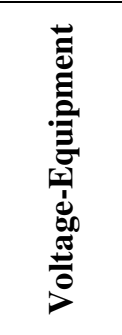 } & \multicolumn{5}{|c|}{$\begin{array}{c}\text { Hazard/Risk Category } \\
\text { Required minimum FR clothing rating }\end{array}$} & \multirow[b]{2}{*}{$\begin{array}{c}\text { Minimum } \\
\text { Approach } \\
\text { Distance } \\
\text { at } 4 \\
\text { cal } / \mathbf{c m}^{2} \\
\text { (ft) }\end{array}$} \\
\hline & & 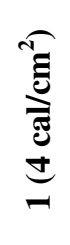 & 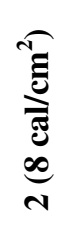 & 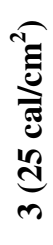 & 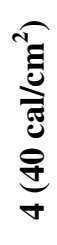 & 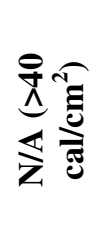 & \\
\hline Bus 1 & $138 \mathrm{kV}$ & & & $\mathrm{X}$ & & & 16.96 \\
\hline Bus 2 & $138 \mathrm{kV}$ & & & $\mathrm{X}$ & & & 14.78 \\
\hline Bus 3 & $138 \mathrm{kV}$ & & & $X$ & & & 12.13 \\
\hline Bus 4 & $138 \mathrm{kV}$ & & & $\mathrm{X}$ & & & 13.71 \\
\hline Bus 5 & $138 \mathrm{kV}$ & & & $\mathrm{X}$ & & & 13.92 \\
\hline Bus 6 & $138 \mathrm{kV}$ & & & & $X$ & & 20.63 \\
\hline Bus 7 & $138 \mathrm{kV}$ & & & $\mathrm{X}$ & & & 18.05 \\
\hline Bus 8 & $138 \mathrm{kV}$ & & & $\mathrm{X}$ & & & 20.09 \\
\hline Bus 9 & $138 \mathrm{kV}$ & & & & $\mathrm{X}$ & & 20.52 \\
\hline Bus 10 & $138 \mathrm{kV}$ & & & & $\mathrm{X}$ & & 20.72 \\
\hline
\end{tabular}




\section{Appendix E Discontinuity of Voltage Levels}

\section{E.1 Voltage Level Test System}

\begin{tabular}{|c|c|c|}
\hline \multicolumn{3}{|c|}{ System 1} \\
\hline Relay 1 -600/5 & Relay 2 - 400/5 & \\
\hline & $\rightarrow$ Load $=2.6 \mathrm{MW}$ & $\rightarrow$ Load $=9 \mathrm{MW}$ \\
\hline $\begin{array}{l}\text { Bus 1 } \\
14.9 \mathrm{kV}\end{array}$ & $\begin{array}{l}\text { Bus } 2 \\
14.9 \mathrm{kV}\end{array}$ & $\begin{array}{l}\text { Bus } 3 \\
14.9 \mathrm{kV}\end{array}$ \\
\hline & System 2 & \\
\hline Relay 1 - 600/5 & Relay 2 - 400/5 & 」 \\
\hline & $\rightarrow$ Load $=2.6 \mathrm{MW}$ & $\longrightarrow$ Load $=9 \mathrm{MW}$ \\
\hline $\begin{array}{l}\text { Bus } 1 \\
15.1 \mathrm{kV}\end{array}$ & $\begin{array}{l}\text { Bus 2 } \\
15.1 \mathrm{kV}\end{array}$ & $\begin{array}{l}\text { Bus } 3 \\
15.1 \mathrm{kV}\end{array}$ \\
\hline
\end{tabular}

Figure E.1: Voltage level test systems

\section{E.2 ASPEN® Results System 1, 51 Contact}

Bus $=$ Bus $314.9 \mathrm{kV}$

Equipment category $=$ Open Air

Grounded $=$ Yes

Enclosed $=$ No

Breaker interrupting time $($ cycles $)=4.0$

Working distance (inches) $=27.0$

Conductor gap $(\mathrm{mm})=50.8$

Bolted 3PH fault current $(\mathrm{kA})=13.962$

Arcing current $(\mathrm{kA})=13.497$

Interrupting device $=\left[\begin{array}{llll}\mathrm{OC} \text { relay }\end{array}\right]$ System 1 Bus 2 on $\quad 0$ Bus $2 \quad 14.9 \mathrm{kV}-\quad 0$ Bus $3 \quad 14.9 \mathrm{kV} 1$

L Line 2

Clearing time (seconds) $=0.11$

Incident energy $(\mathrm{cal} / \mathrm{cm} 2)=1.01$

Interrupting device $@ 85 \%$ current $=[\mathrm{OC}$ relay $]$ System 1 Bus 2 on $\quad 0$ Bus $2 \quad 14.9 \mathrm{kV}-\quad 0$ Bus 3 $14.9 \mathrm{kV} 1 \mathrm{~L}$ Line

Clearing time @85\% current (seconds) $=0.11$

Incident energy @85\% current $(\mathrm{cal} / \mathrm{cm} 2)=0.85$

Required PPE cat. per NFPA 70E $=0$

PPE cat. 1 flash hazard boundary (inches) $=24.7$

PPE cat. 2 flash hazard boundary (inches) $=12.1$

PPE cat. 3 flash hazard boundary (inches) $=9.6$

PPE cat. 4 flash hazard boundary (inches) $=5.4$

Bus $=$ Bus $214.9 \mathrm{kV}$

Equipment category $=$ Open Air

Grounded $=$ Yes

Enclosed $=$ No 
Breaker interrupting time $($ cycles $)=4.0$

Working distance (inches) $=27.0$

Conductor gap $(\mathrm{mm})=50.8$

Bolted 3PH fault current $(\mathrm{kA})=19.358$

Arcing current $(\mathrm{kA})=18.614$

Interrupting device $=\left[\begin{array}{lllll}\mathrm{OC} \text { relay }\end{array}\right]$ System 1 Bus 1 on $\quad 0$ Bus $1 \quad 14.9 \mathrm{kV}-\quad 0$ Bus $2 \quad 14.9 \mathrm{kV} 1$

L Line 1

Clearing time (seconds) $=0.42$

Incident energy $(\mathrm{cal} / \mathrm{cm} 2)=5.50$

Interrupting device @ 85\% current $=$ [OC relay $]$ System 1 Bus 1 on

$14.9 \mathrm{kV} 1 \mathrm{~L}$ Line

Clearing time @ $85 \%$ current (seconds) $=0.43$

Incident energy @85\% current $(\mathrm{cal} / \mathrm{cm} 2)=4.75$

Required PPE cat. per NFPA 70E $=2$

PPE cat. 1 flash hazard boundary (inches) $=57.8$

PPE cat. 2 flash hazard boundary (inches) $=28.3$

PPE cat. 3 flash hazard boundary (inches) $=22.4$

PPE cat. 4 flash hazard boundary (inches) $=12.7$

\section{E.3 ASPEN@ Results System 1, 50 Contact}

Bus $=$ Bus $314.9 \mathrm{kV}$

Equipment category $=$ Open Air

Grounded $=$ Yes

Enclosed $=$ No

Breaker interrupting time $($ cycles $)=4.0$

Working distance (inches) $=27.0$

Conductor gap $(\mathrm{mm})=50.8$

Bolted 3PH fault current $(\mathrm{kA})=13.962$

Arcing current $(\mathrm{kA})=13.497$

Interrupting device $=\left[\begin{array}{llll}\mathrm{OC} \text { relay }\end{array}\right]$ System 1 Bus 2 on $\quad 0$ Bus $2 \quad 14.9 \mathrm{kV}-\quad 0$ Bus $3 \quad 14.9 \mathrm{kV} 1$

L Line 2

Clearing time (seconds) $=0.07$

Incident energy $(\mathrm{cal} / \mathrm{cm} 2)=0.62$

Interrupting device @85\% current $=[\mathrm{OC}$ relay $]$ System 1 Bus 2 on $\quad 0$ Bus $2 \quad 14.9 \mathrm{kV}-\quad 0$ Bus 3 $14.9 \mathrm{kV} 1 \mathrm{~L}$ Line

Clearing time @85\% current (seconds) $=0.07$

Incident energy @85\% current $(\mathrm{cal} / \mathrm{cm} 2)=0.52$

Required PPE cat. per NFPA 70E $=0$

PPE cat. 1 flash hazard boundary (inches) $=19.4$

PPE cat. 2 flash hazard boundary (inches) $=9.5$

PPE cat. 3 flash hazard boundary (inches) $=7.5$

PPE cat. 4 flash hazard boundary (inches) $=4.3$

Bus $=$ Bus $214.9 \mathrm{kV}$

Equipment category $=$ Open Air

Grounded $=$ Yes

Enclosed $=$ No

Breaker interrupting time $($ cycles $)=4.0$

Working distance (inches) $=27.0$

Conductor gap $(\mathrm{mm})=50.8$ 
Bolted 3PH fault current $(\mathrm{kA})=19.358$

Arcing current $(\mathrm{kA})=18.614$

Interrupting device $=\left[\begin{array}{lllll}\mathrm{OC} \text { relay }\end{array}\right]$ System 1 Bus 1 on $\quad 0$ Bus $1 \quad 14.9 \mathrm{kV}-\quad 0$ Bus $2 \quad 14.9 \mathrm{kV} 1$

L Line 1

Clearing time (seconds) $=0.07$

Incident energy $(\mathrm{cal} / \mathrm{cm} 2)=0.88$

Interrupting device @85\% current $=[\mathrm{OC}$ relay $]$ System 1 Bus 1 on $\quad 0$ Bus $1 \quad 14.9 \mathrm{kV}-\quad 0$ Bus 2 $14.9 \mathrm{kV} 1 \mathrm{~L}$ Line

Clearing time @ 85\% current (seconds)=0.07

Incident energy @ $85 \%$ current $(\mathrm{cal} / \mathrm{cm} 2)=0.74$

Required PPE cat. per NFPA 70E $=0$

PPE cat. 1 flash hazard boundary (inches) $=23.1$

PPE cat. 2 flash hazard boundary (inches) $=11.3$

PPE cat. 3 flash hazard boundary (inches) $=9.0$

PPE cat. 4 flash hazard boundary (inches) $=5.1$

\section{E.4 ASPEN® Results System 2, 51 Contact}

Bus $=$ Bus $315.1 \mathrm{kV}$

Equipment category $=$ Open Air

Grounded $=$ Yes

Enclosed $=$ No

Breaker interrupting time $($ cycles $)=4.0$

Working distance (inches) $=27.0$

Conductor gap $(\mathrm{mm})=50.8$

Bolted 3PH fault current $(\mathrm{kA})=13.777$

Arcing current $(\mathrm{kA})=13.777$

Interrupting device $=\left[\begin{array}{llll}\mathrm{OC} \text { relay }\end{array}\right]$ System 2 Bus 2 on $\quad 0$ Bus $2 \quad 15.1 \mathrm{kV}-\quad 0$ Bus $3 \quad 15.1 \mathrm{kV} 1$

L Line 2

Clearing time (seconds) $=0.11$

Incident energy $(\mathrm{cal} / \mathrm{cm} 2)=24.40$

Interrupting device @ 85\% current $=[\mathrm{OC}$ relay $]$ System 2 Bus 2 on $\quad 0$ Bus $2 \quad 15.1 \mathrm{kV}-\quad 0$ Bus 3 $15.1 \mathrm{kV} 1 \mathrm{~L}$ Line

Clearing time @ $85 \%$ current $($ seconds $)=0.11$

Incident energy @85\% current $(\mathrm{cal} / \mathrm{cm} 2)=20.93$

Required PPE cat. per NFPA 70E $=3$

PPE cat. 1 flash hazard boundary (inches) $=121.7$

PPE cat. 2 flash hazard boundary (inches) $=59.6$

PPE cat. 3 flash hazard boundary (inches) $=47.2$

PPE cat. 4 flash hazard boundary (inches) $=26.7$

Bus $=$ Bus $215.1 \mathrm{kV}$

Equipment category $=$ Open Air

Grounded $=$ Yes

Enclosed $=$ No

Breaker interrupting time (cycles) $=4.0$

Working distance (inches) $=27.0$

Conductor gap $(\mathrm{mm})=50.8$

Bolted 3PH fault current $(\mathrm{kA})=19.101$

Arcing current $(\mathrm{kA})=19.101$ 
Interrupting device $=[\mathrm{OC}$ relay $]$ System 2 Bus 1 on $\quad 0$ Bus $1 \quad 15.1 \mathrm{kV}-\quad 0$ Bus $2 \quad 15.1 \mathrm{kV} 1$ L Line 1

Clearing time $($ seconds $)=0.41$

Incident energy $(\mathrm{cal} / \mathrm{cm} 2)=130.16$

Interrupting device @ 85\% current = [OC relay $]$ System 2 Bus 1 on $\quad 0$ Bus $1 \quad 15.1 \mathrm{kV}-\quad 0$ Bus 2 $15.1 \mathrm{kV} 1 \mathrm{~L}$ Line

Clearing time @ $85 \%$ current (seconds) $=0.43$

Incident energy@85\% current $(\mathrm{cal} / \mathrm{cm} 2)=113.79$

Required PPE cat. per NFPA 70E = N/A

PPE cat. 1 flash hazard boundary (inches) $=281.2$

PPE cat. 2 flash hazard boundary (inches) $=137.8$

PPE cat. 3 flash hazard boundary (inches) $=108.9$

PPE cat. 4 flash hazard boundary (inches) $=61.6$

\section{E.5 ASPEN® Results Using 50 and 51 Contacts}

Table E.1: Incident energy level based on trip contact selected

\begin{tabular}{|c|c|c|c|c|c|c|c|c|}
\hline \multirow[b]{2}{*}{ Station } & \multirow{2}{*}{ 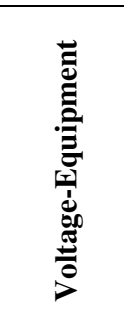 } & \multicolumn{6}{|c|}{$\begin{array}{c}\text { Hazard/Risk Category } \\
\text { Required minimum FR clothing rating }\end{array}$} & \multirow[b]{2}{*}{$\begin{array}{c}\text { Minimum } \\
\text { Approach } \\
\text { Distance a } \\
4 \text { cal } / \mathrm{cm}^{2} \\
\text { (ft) }\end{array}$} \\
\hline & & 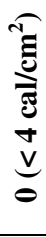 & 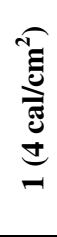 & 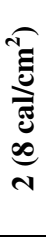 & 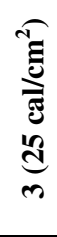 & 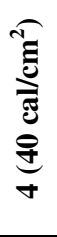 & 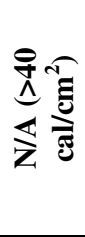 & \\
\hline $\begin{array}{c}\text { System } 1 \\
\text { Bus } 250 \\
\text { Contact }\end{array}$ & $14.9 \mathrm{kV}$ & $X$ & & & & & & 1.93 \\
\hline $\begin{array}{c}\text { System } 1 \\
\text { Bus } 251 \\
\text { Contact }\end{array}$ & $14.9 \mathrm{kV}$ & & & $X$ & & & & 4.82 \\
\hline $\begin{array}{c}\text { System } 1 \\
\text { Bus } 350 \\
\text { Contact }\end{array}$ & $14.9 \mathrm{kV}$ & $\mathrm{X}$ & & & & & & 1.62 \\
\hline $\begin{array}{c}\text { System } 1 \\
\text { Bus } 351 \\
\text { Contact }\end{array}$ & $14.9 \mathrm{kV}$ & $X$ & & & & & & 2.06 \\
\hline
\end{tabular}




\section{E.6 ASPEN® Results Systems 1 \& 2}

Table E.2: Voltage level system comparisons

\begin{tabular}{|c|c|c|c|c|c|c|c|c|}
\hline \multirow[b]{2}{*}{ Station } & \multirow{2}{*}{ 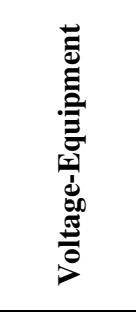 } & \multicolumn{6}{|c|}{$\begin{array}{c}\text { Hazard/Risk Category } \\
\text { Required minimum FR clothing rating }\end{array}$} & \multirow[b]{2}{*}{$\begin{array}{c}\text { Minimum } \\
\text { Approach } \\
\text { Distance a } \\
\mathbf{4} \text { cal/cm } \\
\text { (ft) }\end{array}$} \\
\hline & & 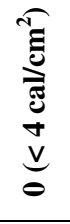 & 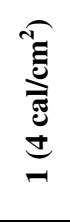 & 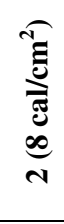 & 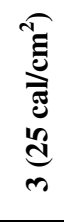 & 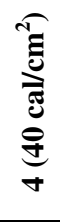 & 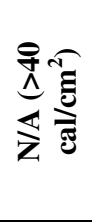 & \\
\hline $\begin{array}{l}\text { System } 1 \\
\text { Bus } 2 \\
\end{array}$ & $14.9 \mathrm{kV}$ & & & $X$ & & & & 4.82 \\
\hline $\begin{array}{l}\text { System } 2 \\
\text { Bus } 2 \\
\end{array}$ & $15.1 \mathrm{kV}$ & & & & & & X & 23.43 \\
\hline $\begin{array}{l}\text { System } 1 \\
\text { Bus } 3\end{array}$ & $14.9 \mathrm{kV}$ & $X$ & & & & & & 2.06 \\
\hline $\begin{array}{l}\text { System } 2 \\
\text { Bus } 3 \\
\end{array}$ & $15.1 \mathrm{kV}$ & & & & $X$ & & & 10.14 \\
\hline
\end{tabular}




\section{Appendix F Energy Plot Data}

Table F.1: U.S. U2 inverse time overcurrent curve data

\begin{tabular}{|c|c|c|c|c|}
\hline \multirow[b]{2}{*}{$\begin{array}{l}\text { Multiple of } \\
\text { Pickup }\end{array}$} & \multicolumn{4}{|c|}{ Trip Time (s) } \\
\hline & $\begin{array}{c}\text { Time Dial } \\
0.5\end{array}$ & Time Dial 2 & Time Dial 5 & $\begin{array}{c}\text { Time Dial } \\
11\end{array}$ \\
\hline 1.5 & 2.47 & 9.88 & 24.7 & 54.34 \\
\hline 2 & 1.081666667 & 4.3266666667 & 10.81666667 & 23.796666667 \\
\hline 3 & 0.461875 & 1.8475 & 4.61875 & 10.16125 \\
\hline 4 & 0.288333333 & 1.153333333 & 2.883333333 & 6.343333333 \\
\hline 5 & 0.213958333 & 0.855833333 & 2.139583333 & 4.707083333 \\
\hline 6 & 0.175 & 0.7 & 1.75 & 3.85 \\
\hline 7 & 0.151979167 & 0.607916667 & 1.519791667 & 3.343541667 \\
\hline 8 & 0.137222222 & 0.548888889 & 1.372222222 & 3.018888889 \\
\hline 9 & 0.1271875 & 0.50875 & 1.271875 & 2.798125 \\
\hline 10 & 0.120050505 & 0.48020202 & 1.200505051 & 2.641111111 \\
\hline 11 & 0.114791667 & 0.459166667 & 1.147916667 & 2.525416667 \\
\hline 12 & 0.110804196 & 0.443216783 & 1.108041958 & 2.437692308 \\
\hline 13 & 0.107708333 & 0.430833333 & 1.077083333 & 2.369583333 \\
\hline 14 & 0.10525641 & 0.421025641 & 1.052564103 & 2.315641026 \\
\hline 15 & 0.10328125 & 0.413125 & 1.0328125 & 2.2721875 \\
\hline 16 & 0.101666667 & 0.406666667 & 1.016666667 & 2.2366666667 \\
\hline 17 & 0.100329861 & 0.401319444 & 1.003298611 & 2.207256944 \\
\hline 18 & 0.099210526 & 0.396842105 & 0.992105263 & 2.182631579 \\
\hline 19 & 0.098263889 & 0.393055556 & 0.982638889 & 2.161805556 \\
\hline 20 & 0.09745614 & 0.389824561 & 0.974561404 & 2.144035088 \\
\hline 21 & 0.096761364 & 0.387045455 & 0.967613636 & 2.12875 \\
\hline 22 & 0.09615942 & 0.384637681 & 0.961594203 & 2.115507246 \\
\hline 23 & 0.09563447 & 0.382537879 & 0.956344697 & 2.103958333 \\
\hline 24 & 0.095173913 & 0.380695652 & 0.95173913 & 2.093826087 \\
\hline 25 & 0.094767628 & 0.379070513 & 0.947676282 & 2.084887821 \\
\hline 26 & 0.094407407 & 0.37762963 & 0.944074074 & 2.076962963 \\
\hline 27 & 0.094086538 & 0.376346154 & 0.940865385 & 2.069903846 \\
\hline 28 & 0.093799489 & 0.375197957 & 0.937994891 & 2.063588761 \\
\hline 29 & 0.093541667 & 0.374166667 & 0.935416667 & 2.057916667 \\
\hline 30 & 0.093309232 & 0.37323693 & 0.933092325 & 2.052803115 \\
\hline 31 & 0.093098958 & 0.372395833 & 0.930989583 & 2.048177083 \\
\hline 32 & 0.092908113 & 0.371632454 & 0.929081134 & 2.043978495 \\
\hline 33 & 0.092734375 & 0.3709375 & 0.92734375 & 2.04015625 \\
\hline 34 & 0.092575758 & 0.37030303 & 0.925757576 & 2.0366666667 \\
\hline 35 & 0.092430556 & 0.369722222 & 0.924305556 & 2.033472222 \\
\hline 36 & 0.092297297 & 0.369189189 & 0.922972973 & 2.030540541 \\
\hline 37 & 0.092174708 & 0.36869883 & 0.921747076 & 2.027843567 \\
\hline 38 & 0.092061677 & 0.368246708 & 0.920616771 & 2.025356895 \\
\hline 39 & 0.091957237 & 0.367828947 & 0.919572368 & 2.023059211 \\
\hline 40 & 0.091860538 & 0.367442151 & 0.918605378 & 2.020931832 \\
\hline
\end{tabular}




\begin{tabular}{|c|c|c|c|c|}
\hline 41 & 0.091770833 & 0.367083333 & 0.917708333 & 2.018958333 \\
\hline 42 & 0.091687465 & 0.366749858 & 0.916874645 & 2.01712422 \\
\hline 43 & 0.091609848 & 0.366439394 & 0.916098485 & 2.015416667 \\
\hline 44 & 0.091537468 & 0.366149871 & 0.915374677 & 2.013824289 \\
\hline 45 & 0.091469862 & 0.365879447 & 0.914698617 & 2.012336957 \\
\hline 46 & 0.091406619 & 0.365626478 & 0.914066194 & 2.010945626 \\
\hline 47 & 0.091347373 & 0.365389493 & 0.913473732 & 2.00964221 \\
\hline 48 & 0.091291793 & 0.365167173 & 0.912917933 & 2.008419453 \\
\hline 49 & 0.091239583 & 0.364958333 & 0.912395833 & 2.007270833 \\
\hline 50 & 0.091190476 & 0.364761905 & 0.911904762 & 2.006190476 \\
\hline 51 & 0.091144231 & 0.364576923 & 0.911442308 & 2.005173077 \\
\hline 52 & 0.091100629 & 0.364402516 & 0.911006289 & 2.004213836 \\
\hline 53 & 0.091059473 & 0.364237892 & 0.910594729 & 2.003308405 \\
\hline 54 & 0.091020583 & 0.364082333 & 0.910205832 & 2.00245283 \\
\hline 55 & 0.090983796 & 0.363935185 & 0.909837963 & 2.001643519 \\
\hline 56 & 0.090948963 & 0.363795853 & 0.909489633 & 2.000877193 \\
\hline 57 & 0.090915948 & 0.363663793 & 0.909159483 & 2.000150862 \\
\hline 58 & 0.090884627 & 0.363538507 & 0.908846268 & 1.99946179 \\
\hline 59 & 0.090854885 & 0.36341954 & 0.908548851 & 1.998807471 \\
\hline 60 & 0.090826619 & 0.363306474 & 0.908266185 & 1.998185607 \\
\hline 61 & 0.090799731 & 0.363198925 & 0.907997312 & 1.997594086 \\
\hline 62 & 0.090774135 & 0.363096539 & 0.907741348 & 1.997030965 \\
\hline 63 & 0.090749748 & 0.362998992 & 0.90749748 & 1.996494456 \\
\hline 64 & 0.090726496 & 0.362905983 & 0.907264957 & 1.995982906 \\
\hline 65 & 0.090704309 & 0.362817235 & 0.907043087 & 1.995494792 \\
\hline 66 & 0.090683123 & 0.362732491 & 0.906831228 & 1.995028703 \\
\hline 67 & 0.090662879 & 0.362651515 & 0.906628788 & 1.994583333 \\
\hline 68 & 0.090643522 & 0.362574086 & 0.906435215 & 1.994157474 \\
\hline 69 & 0.090625 & 0.3625 & 0.90625 & 1.99375 \\
\hline 70 & 0.090607267 & 0.362429067 & 0.906072668 & 1.993359869 \\
\hline 71 & 0.090590278 & 0.362361111 & 0.905902778 & 1.992986111 \\
\hline 72 & 0.090573992 & 0.362295968 & 0.905739919 & 1.992627822 \\
\hline 73 & 0.090558371 & 0.362233483 & 0.905583709 & 1.992284159 \\
\hline 74 & 0.090543379 & 0.362173516 & 0.90543379 & 1.991954338 \\
\hline 75 & 0.090528983 & 0.362115932 & 0.905289829 & 1.991637624 \\
\hline 76 & 0.090515152 & 0.362060606 & 0.905151515 & 1.991333333 \\
\hline 77 & 0.090501856 & 0.362007422 & 0.905018556 & 1.991040823 \\
\hline 78 & 0.090489068 & 0.361956272 & 0.904890679 & 1.990759494 \\
\hline 79 & 0.090476763 & 0.361907051 & 0.904767628 & 1.990488782 \\
\hline 80 & 0.090464916 & 0.361859666 & 0.904649164 & 1.990228161 \\
\hline 81 & 0.090453506 & 0.361814024 & 0.904535061 & 1.989977134 \\
\hline 82 & 0.090442511 & 0.361770043 & 0.904425108 & 1.989735237 \\
\hline 83 & 0.090431911 & 0.361727642 & 0.904319106 & 1.989502033 \\
\hline 84 & 0.090421687 & 0.361686747 & 0.904216867 & 1.989277108 \\
\hline 85 & 0.090411822 & 0.361647287 & 0.904118217 & 1.989060078 \\
\hline 86 & 0.090402299 & 0.361609195 & 0.904022989 & 1.988850575 \\
\hline
\end{tabular}




\begin{tabular}{|c|c|c|c|c|}
87 & 0.090393103 & 0.36157241 & 0.903931025 & 1.988648256 \\
\hline 88 & 0.090384218 & 0.361536872 & 0.90384218 & 1.988452796 \\
\hline 89 & 0.090375631 & 0.361502525 & 0.903756313 & 1.988263889 \\
\hline 90 & 0.090367329 & 0.361469317 & 0.903673293 & 1.988081245 \\
\hline 91 & 0.0903593 & 0.361437198 & 0.903592995 & 1.987904589 \\
\hline 92 & 0.09035153 & 0.361406121 & 0.903515302 & 1.987733664 \\
\hline 93 & 0.09034401 & 0.361376041 & 0.903440102 & 1.987568224 \\
\hline 94 & 0.090336729 & 0.361346916 & 0.903367289 & 1.987408036 \\
\hline 95 & 0.090329676 & 0.361318706 & 0.903296764 & 1.987252881 \\
\hline 96 & 0.090322843 & 0.361291373 & 0.903228432 & 1.98710255 \\
\hline 97 & 0.09031622 & 0.361264881 & 0.903162202 & 1.986956845 \\
\hline 98 & 0.090309799 & 0.361239196 & 0.90309799 & 1.986815578 \\
\hline 99 & 0.090303571 & 0.361214286 & 0.903035714 & 1.986678571 \\
\hline 100 & 0.09029753 & 0.361190119 & 0.902975298 & 1.986545655 \\
\hline
\end{tabular}

Table F.2: Energy through equipment curve data

\begin{tabular}{|c|c|c|c|c|}
\hline \multirow[b]{2}{*}{$\begin{array}{l}\text { Multiple of } \\
\text { Pickup }\end{array}$} & \multicolumn{4}{|c|}{ Energy $(\mathrm{J})$} \\
\hline & $\begin{array}{c}\text { Time Dial } \\
0.5\end{array}$ & $\begin{array}{l}\text { Time } \\
\text { Dial } 2\end{array}$ & $\begin{array}{c}\text { Time } \\
\text { Dial } 5\end{array}$ & $\begin{array}{c}\text { Time Dial } \\
11\end{array}$ \\
\hline 0.694444444 & 2.95689092 & 11.82756 & 29.56891 & 65.0516 \\
\hline 0.722222222 & 3.19814132 & 12.79257 & 31.98141 & 70.359109 \\
\hline 0.75 & 3.44885252 & 13.79541 & 34.48853 & 75.874755 \\
\hline 0.777777778 & 3.70902452 & 14.8361 & 37.09025 & 81.598539 \\
\hline 0.805555556 & 3.97865732 & 15.91463 & 39.78657 & 87.530461 \\
\hline 0.833333333 & 4.25775092 & 17.031 & 42.57751 & 93.67052 \\
\hline 0.861111111 & 4.54630532 & 18.18522 & 45.46305 & 100.01872 \\
\hline 0.8888888889 & 4.84432052 & 19.37728 & 48.44321 & 106.57505 \\
\hline 0.9166666667 & 5.15179652 & 20.60719 & 51.51797 & 113.33952 \\
\hline 0.944444444 & 5.46873332 & 21.87493 & 54.68733 & 120.31213 \\
\hline 0.972222222 & 5.79513092 & 23.18052 & 57.95131 & 127.49288 \\
\hline 1 & 6.13098932 & 24.52396 & 61.30989 & 134.88176 \\
\hline 1.027777778 & 6.47630852 & 25.90523 & 64.76309 & 142.47879 \\
\hline 1.0555555566 & 6.83108852 & 27.32435 & 68.31089 & 150.28395 \\
\hline 1.083333333 & 7.19532932 & 28.78132 & 71.95329 & 158.29724 \\
\hline 1.111111111 & 7.56903092 & 30.27612 & 75.69031 & 166.51868 \\
\hline 1.138888889 & 7.95219332 & 31.80877 & 79.52193 & 174.94825 \\
\hline 1.1666666667 & 8.34481652 & 33.37927 & 83.44817 & 183.58596 \\
\hline 1.194444444 & 8.74690052 & 34.9876 & 87.46901 & 192.43181 \\
\hline 1.222222222 & 9.15844532 & 36.63378 & 91.58445 & 201.4858 \\
\hline 1.25 & 9.57945092 & 38.3178 & 95.79451 & 210.74792 \\
\hline 1.2777777778 & 10.0099173 & 40.03967 & 100.0992 & 220.21818 \\
\hline 1.305555556 & 10.4498445 & 41.79938 & 104.4984 & 229.89658 \\
\hline 1.333333333 & 10.8992325 & 43.59693 & 108.9923 & 239.78312 \\
\hline 1.361111111 & 11.3580813 & 45.43233 & 113.5808 & 249.87779 \\
\hline 1.3888888889 & 11.8263909 & 47.30556 & 118.2639 & 260.1806 \\
\hline 1.416666667 & 12.3041613 & 49.21665 & 123.0416 & 270.69155 \\
\hline
\end{tabular}




\begin{tabular}{|c|c|c|c|c|}
\hline 1.444444444 & 12.7913925 & 51.16557 & 127.9139 & 281.41064 \\
\hline 1.472222222 & 13.2880845 & 53.15234 & 132.8808 & 292.33786 \\
\hline 1.5 & 13.7942373 & 55.17695 & 137.9424 & 303.47322 \\
\hline 1.527777778 & 14.3098509 & 57.2394 & 143.0985 & 314.81672 \\
\hline 1.555555556 & 14.8349253 & 59.3397 & 148.3493 & 326.36836 \\
\hline 1.583333333 & 15.3694605 & 61.47784 & 153.6946 & 338.12813 \\
\hline 1.611111111 & 15.9134565 & 63.65383 & 159.1346 & 350.09604 \\
\hline 1.638888889 & 16.4669133 & 65.86765 & 164.6691 & 362.27209 \\
\hline 1.6666666667 & 17.0298309 & 68.11932 & 170.2983 & 374.65628 \\
\hline 1.694444444 & 17.6022093 & 70.40884 & & 387.2486 \\
\hline 1.722222222 & 18.1840485 & 72.73619 & 181.8405 & 400.04907 \\
\hline 1.75 & 18.7753485 & 75.10139 & 187.7535 & 413.05767 \\
\hline 1.777777778 & 19.3761093 & 77.50444 & 193.7611 & 426.2744 \\
\hline 1.805555556 & 19.9863309 & 79.9 & 199.8 & 439.6 \\
\hline 1.833333333 & 20.6060133 & 82.42405 & 206.0601 & 453.33229 \\
\hline 1.861111111 & 21.23 & 84.9 & 212 & 7344 \\
\hline 1.888888889 & 21.8737605 & 87.49504 & 218.7376 & 481.22273 \\
\hline 1.9166666667 & 22.5218253 & 90.0873 & 225.2183 & 495.48016 \\
\hline 1.944444444 & 23.17 & 92.7174 & 231 & 4572 \\
\hline 1.972222222 & 23.8 & & & 1942 \\
\hline 2 & 24.5227845 & 98.09114 & 245.2278 & 539.50126 \\
\hline 2.027777778 & 25.2086925 & 100.8348 & 252.0869 & 9124 \\
\hline 2.055555556 & 25.9 & & 406 & 8935 \\
\hline 2.083333333 & 26.6088909 & 106.4356 & 266.0889 & 585.3956 \\
\hline 2.111111111 & 27.3231813 & 109.2927 & 273.2318 & 601.10999 \\
\hline 2.138888889 & 28.0469325 & 112.1877 & 280.4693 & 617.03252 \\
\hline 2.1666666667 & 28.7801445 & 115.1206 & 287.8014 & 633.16318 \\
\hline 2.194444444 & 29.5228173 & 118.0913 & 295.2282 & 649.50198 \\
\hline 2.222222222 & 30.2749509 & 121.0998 & 302.7495 & 666.04892 \\
\hline 2.25 & 31.0365453 & 124.1462 & 310.3655 & 682.804 \\
\hline 2.277777778 & 31.8076005 & 127.2304 & 318.076 & 699.76721 \\
\hline 2.305555556 & 32.5881165 & 130.3525 & 325.8812 & 716.93856 \\
\hline 2.333333333 & 33.3780933 & 133.5124 & 333.7809 & 734.31805 \\
\hline 2.361111111 & 34.1775309 & 136.7101 & 341.7753 & 751.90568 \\
\hline 2.388888889 & 34.9864293 & 139.9457 & 349.8643 & 769.70144 \\
\hline 2.416666667 & 35.8047885 & 143.2192 & 358.0479 & 787.70535 \\
\hline 2.444444444 & 36.6326085 & 146.5304 & 366.3261 & 805.91739 \\
\hline 2.472222222 & 37.4698893 & 149.8796 & 374.6989 & 824.33756 \\
\hline 2.5 & 38.3166309 & 153.2665 & 383.1663 & 842.96588 \\
\hline 2.527777778 & 39.1728333 & 156.6913 & 391.7283 & 861.80233 \\
\hline 2.555555556 & 40.0384965 & 160.154 & 400.385 & 880.84692 \\
\hline 2.583333333 & 40.9136205 & 163.6545 & 409.1362 & 900.09965 \\
\hline 2.611111111 & 41.7982053 & 167.1928 & 417.9821 & 919.56052 \\
\hline 2.638888889 & 42.6922509 & 170.769 & 426.9225 & 939.22952 \\
\hline 2.6666666667 & 43.5957573 & & 435.9576 & 959.10666 \\
\hline 2.694444444 & 44.5087245 & 178.0349 & 445.0872 & 979.19194 \\
\hline
\end{tabular}




\begin{tabular}{|c|c|c|c|c|}
\hline 2.722222222 & 45.4311525 & 181.7246 & 454.3115 & 999.48536 \\
\hline 2.75 & 46.3630413 & 185.4522 & 463.6304 & 1019.9869 \\
\hline 2.777777778 & 47.3043909 & 189.2176 & 473.0439 & 1040.6966 \\
\hline 2.805555556 & 48.2552013 & 193.0208 & 482.552 & 1061.6144 \\
\hline 2.833333333 & 49.2154725 & 196.8619 & 492.1547 & 1082.7404 \\
\hline 2.861111111 & 50.1852045 & 200.7408 & 501.852 & 1104.0745 \\
\hline 2.888888889 & 51.1643973 & 204.6576 & 511.644 & 1125.6167 \\
\hline 2.916666667 & 52.1530509 & 208.6122 & 521.5305 & 1147.3671 \\
\hline 2.944444444 & 53.1511653 & 212.6047 & 531.5117 & 1169.3256 \\
\hline 2.972222222 & 54.1587405 & 216.635 & 541.5874 & 1191.4923 \\
\hline 3 & 55.1757765 & 220.7031 & 551.7578 & 1213.8671 \\
\hline 3.027777778 & 56.2022733 & 224.8091 & 562.0227 & 1236.45 \\
\hline 3.055555556 & 57.2382309 & 228.9529 & 572.3823 & 1259.2411 \\
\hline 3.083333333 & 58.2 & & & 2403 \\
\hline 3.111111111 & 59.3385285 & 237.3541 & 593.3853 & 1305.4476 \\
\hline 3.138888889 & 60.4028685 & 241.6115 & 604.0287 & 1328.8631 \\
\hline 3.166666667 & & & & 867 \\
\hline 3.194444444 & 62.559 & 250. & 625. & 1376.3185 \\
\hline 3.222222222 & 63.6526533 & 254.6106 & 636.5265 & 1400.3584 \\
\hline 3.25 & 64.75 & 259. & 484 & 5064 \\
\hline 3.277777778 & 65.8664805 & 263.4659 & 658.6648 & 1449.0626 \\
\hline 3.305555556 & 66.9875853 & 267.9503 & 669.8759 & 1473.7269 \\
\hline 3.333333333 & 68.11 & 272.4726 & 681 & 5993 \\
\hline 3.361111111 & 69.2581773 & 277.0327 & 692. & 1523.6799 \\
\hline 3.388888889 & 70.4076645 & 281.6307 & 9766 & 1548.9686 \\
\hline 3.416666667 & 71.5666125 & 286.2665 & 715.6661 & 1574.4655 \\
\hline 3.444444444 & 72.7 & 290.9401 & 3502 & 1600.1705 \\
\hline 3.472222222 & 73.9128909 & 295.6516 & 739.1 & 1626.0836 \\
\hline 3.5 & 75.1002213 & 300.4009 & 751.0022 & 1652.2049 \\
\hline 3.527777778 & 0125 & 305.1881 & 762.9701 & 1678.5343 \\
\hline 3.555555556 & 77.5032645 & 310.0131 & 775.0326 & 1705.0718 \\
\hline 3.583333333 & 78.7189773 & 314.8759 & 787.1898 & 1731.8175 \\
\hline 3.611111111 & 79.9441509 & 319.7766 & 799.4415 & 1758.7713 \\
\hline 3.638888889 & 81.1787853 & 324.7151 & 811.7879 & 1785.9333 \\
\hline 3.666666667 & 82.4228805 & 329.6915 & 824.2288 & 1813.3034 \\
\hline 3.694444444 & 83.6764365 & 334.7057 & 836.7644 & 1840.8816 \\
\hline 3.722222222 & 84.9394533 & 339.7578 & 849.3945 & 1868.668 \\
\hline 3.75 & 86.2119309 & 344.8477 & 862.1193 & 1896.6625 \\
\hline 3.777777778 & 87.4938693 & 349.9755 & 874.9387 & 1924.8651 \\
\hline 3.805555556 & 88.7852685 & 355.1411 & 887.8527 & 1953.2759 \\
\hline 3.833333333 & 90.0861285 & 360.3445 & 900.8613 & 1981.8948 \\
\hline 3.861111111 & 91.3964493 & 365.5858 & 913.9645 & 2010.7219 \\
\hline 3.888888889 & 92.7162309 & 370.8649 & 927.1623 & 2039.7571 \\
\hline 3.916666667 & 94.0454733 & 376.1819 & 940.4547 & 2069.0004 \\
\hline 3.944444444 & 95.3841765 & 381.5367 & 953.8418 & 2098.4519 \\
\hline 3.972222222 & 96.7323405 & 386.9294 & 967.3234 & 2128.1115 \\
\hline
\end{tabular}




\begin{tabular}{|c|c|c|c|c|}
\hline 4 & 98.0899653 & 392.3599 & 980.8997 & 2157.9792 \\
\hline 4.027777778 & 99.4570509 & 397.8282 & 994.5705 & 2188.0551 \\
\hline 4.055555556 & 100.833597 & 403.3344 & 1008.336 & 2218.3391 \\
\hline 4.083333333 & 102.219605 & 408.8784 & 1022.196 & 2248.8313 \\
\hline 4.111111111 & 103.615073 & 414.4603 & 1036.151 & 2279.5316 \\
\hline 4.138888889 & 105.020001 & 420.08 & 1050.2 & 2310.44 \\
\hline 4.166666667 & 106.434391 & 425.7376 & 1064.344 & 2341.5566 \\
\hline 4.194444444 & 107.858241 & 431.433 & 1078.582 & 2372.8813 \\
\hline 4.222222222 & 109.291553 & 437.1662 & 1092.916 & 2404.4142 \\
\hline 4.25 & 110.734325 & 442.9373 & 1107.343 & 2436.1551 \\
\hline 4.277777778 & 112.186557 & 448.7462 & 1121.866 & 2468.1043 \\
\hline 4.305555556 & 113.648251 & 454.593 & 1136.483 & 2500.2615 \\
\hline 4.333333333 & 115.119405 & 460.4776 & 1151.194 & 269 \\
\hline 4.361111111 & 116.600021 & 466.4001 & 1166 & 2565.2005 \\
\hline 4.388888889 & 118.090097 & 472.3604 & 1180.901 & 2597.9821 \\
\hline 4.416666667 & 119.589633 & 478.3585 & 1195.896 & 2630.9719 \\
\hline 4.444444444 & 121.098631 & 484.3945 & 1210.986 & 2664.1699 \\
\hline 4.472222222 & 122.617089 & 490.4684 & 1226.171 & 2697.576 \\
\hline 4.5 & 124.12 & 496.58 & 1241.45 & 902 \\
\hline 4.527777778 & 125.6 & 502.7296 & 1256.824 & \\
\hline 4.555555556 & 127.229229 & 508.9169 & 1272.292 & 2799.043 \\
\hline 4.583333333 & 128.785531 & 515.1421 & 1287.855 & 2833.2817 \\
\hline 4.611111111 & 130.3 & 521.4052 & 513 & \\
\hline 4.638888889 & 131.926517 & 527.7061 & 1319.265 & 2902.3834 \\
\hline 4.666666667 & 133.511201 & 534.0448 & 1335.112 & 7.2464 \\
\hline 4.694444444 & 135.1 & 540.4214 & 1351.053 & 2.3176 \\
\hline 4.722222222 & 136.708951 & 546.8358 & 1367.09 & 3007.5969 \\
\hline 4.75 & 138.322017 & 553.2881 & 1383.22 & 3043.0844 \\
\hline 4.777777778 & 139.944545 & 559.7782 & 1399.445 & 3078.78 \\
\hline 4.805555556 & 141.576533 & 566.3061 & 1415.765 & 3114.6837 \\
\hline 4.833333333 & 143.217981 & 572.8719 & 1432.18 & 3150.7956 \\
\hline 4.861111111 & 144.868891 & 579.4756 & 1448.689 & 3187.1156 \\
\hline 4.888888889 & 146.529261 & 586.117 & 1465.293 & 3223.6437 \\
\hline 4.916666667 & 148.199093 & 592.7964 & 1481.991 & 3260.38 \\
\hline 4.944444444 & 149.878385 & 599.5135 & 1498.784 & 3297.3245 \\
\hline 4.972222222 & 151.567137 & 606.2685 & 1515.671 & 3334.477 \\
\hline 5 & 153.265351 & 613.0614 & 1532.654 & 3371.8377 \\
\hline 5.027777778 & 154.973025 & 619.8921 & 1549.73 & 3409.4066 \\
\hline 5.055555556 & 156.690161 & 626.7606 & 1566.902 & 3447.1835 \\
\hline 5.083333333 & 158.416757 & 633.667 & 1584.168 & 3485.1686 \\
\hline 5.111111111 & 160.152813 & 640.6113 & 1601.528 & 3523.3619 \\
\hline 5.138888889 & 161.898331 & 647.5933 & 1618.983 & 3561.7633 \\
\hline 5.166666667 & 163.653309 & 654.6132 & 1636.533 & 3600.3728 \\
\hline 5.194444444 & 165.417749 & 661.671 & 1654.177 & 3639.1905 \\
\hline 5.222222222 & 167.191649 & 668.7666 & 1671.916 & 3678.2163 \\
\hline 5.25 & 168.975009 & 675.9 & 1689.75 & 3717.4502 \\
\hline
\end{tabular}




\begin{tabular}{|c|c|c|c|c|}
\hline 5.277777778 & 170.767831 & 683.0713 & 1707.678 & 3756.8923 \\
\hline 5.305555556 & 172.570113 & 690.2805 & 1725.701 & 3796.5425 \\
\hline 5.333333333 & 174.381857 & 697.5274 & 1743.819 & 3836.4008 \\
\hline 5.361111111 & 176.203061 & 704.8122 & 1762.031 & 3876.4673 \\
\hline 5.388888889 & 178.033725 & 712.1349 & 1780.337 & 3916.742 \\
\hline 5.416666667 & 179.873851 & 719.4954 & 1798.739 & 3957.2247 \\
\hline 5.444444444 & 181.723437 & 726.8937 & 1817.234 & 3997.9156 \\
\hline 5.472222222 & 183.582485 & 734.3299 & 1835.825 & 4038.8147 \\
\hline 5.5 & 185.450993 & 741.804 & 1854.51 & 4079.9218 \\
\hline 5.527777778 & 187.328961 & 749.3158 & 873.29 & 4121.2371 \\
\hline 5.555555556 & 189.216391 & 756.8656 & 1892.164 & 4162.7606 \\
\hline 5.583333333 & 191.113281 & 764.4531 & 1911.133 & 4204.4922 \\
\hline 5.611111111 & 193.01 & 772.0785 & 1930.196 & 424 \\
\hline 5.638888889 & 194.9 & 779.7418 & 1949.354 & 4288.5798 \\
\hline 5.666666667 & 196.860717 & 787.4429 & 1968.607 & 4330.9358 \\
\hline 5.694444444 & 198.7 & 795.1818 & & \\
\hline 5.722222222 & 200.7396 & 802.9586 & 2007.396 & 4416.2722 \\
\hline 5.75 & 202.693301 & 810.7732 & 2026.933 & 4459.2526 \\
\hline 5.777777778 & 204.6 & 818.6 & 204 & \\
\hline 5.8055 & 206.6 & 826.516 & 6.29 & \\
\hline 5.833333333 & 208.611031 & 834.4441 & 086.11 & 4589.4427 \\
\hline 5.861111111 & 210.602529 & 842.4101 & 2106.025 & 4633 \\
\hline 5.888888889 & 212.6 & 850.414 & 35 & \\
\hline 5.9166666667 & 214.613909 & 858.4556 & 2146.139 & 4721.506 \\
\hline 5.944444444 & 216.633789 & 866.5352 & 2166. & 4765 \\
\hline 5.972222222 & 218. & 874.6525 & 631 & 5889 \\
\hline 6 & 220.701933 & 882.8077 & 2207.019 & 4855.4425 \\
\hline 6.027777778 & 222.750197 & 891.0008 & 2227.502 & 4900.5043 \\
\hline 6.055555556 & 224.807921 & 899.2317 & 2248.079 & 4945.7743 \\
\hline 6.083333333 & 226.875105 & 907.5004 & 2268.751 & 4991.2523 \\
\hline 6.111111111 & 228.951751 & 915.807 & 2289.518 & 5036.9385 \\
\hline 6.138888889 & 231.037857 & 924.1514 & 2310.379 & 5082.8329 \\
\hline 6.166666667 & 233.133425 & 932.5337 & 2331.334 & 5128.9353 \\
\hline 6.194444444 & 235.238453 & 940.9538 & 2352.385 & 5175.246 \\
\hline 6.222222222 & 237.352941 & 949.4118 & 2373.529 & 5221.7647 \\
\hline 6.25 & 239.476891 & 957.9076 & 2394.769 & 5268.4916 \\
\hline 6.277777778 & 241.610301 & 966.4412 & 2416.103 & 5315.4266 \\
\hline 6.305555556 & 243.753173 & 975.0127 & 2437.532 & 5362.5698 \\
\hline 6.333333333 & 245.905505 & 983.622 & 2459.055 & 5409.9211 \\
\hline 6.361111111 & 248.067297 & 992.2692 & 2480.673 & 5457.4805 \\
\hline 6.388888889 & 250.238551 & 1000.954 & 2502.386 & 5505.2481 \\
\hline 6.416666667 & 252.419265 & 1009.677 & 2524.193 & 5553.2238 \\
\hline 6.444444444 & 254.609441 & 1018.438 & 2546.094 & 5601.4077 \\
\hline 6.472222222 & 256.809077 & 1027.236 & 2568.091 & 5649.7997 \\
\hline 6.5 & 259.018173 & 1036.073 & 2590.182 & 5698.3998 \\
\hline 6.527777778 & 261.236731 & 1044.947 & 2612.367 & 5747.2081 \\
\hline
\end{tabular}




\begin{tabular}{|c|c|c|c|c|}
\hline 6.555555556 & 263.464749 & 1053.859 & 2634.647 & 5796.2245 \\
\hline 6.583333333 & 265.702229 & 1062.809 & 2657.022 & 5845.449 \\
\hline 6.611111111 & 267.949169 & 1071.797 & 2679.492 & 5894.8817 \\
\hline 6.638888889 & 270.205569 & 1080.822 & 2702.056 & 5944.5225 \\
\hline 6.6666666667 & 272.471431 & 1089.886 & 2724.714 & 5994.3715 \\
\hline 6.694444444 & 274.746753 & 1098.987 & 2747.468 & 6044.4286 \\
\hline 6.722222222 & 277.031537 & 1108.126 & 2770.315 & 6094.6938 \\
\hline 6.75 & 279.325781 & 1117.303 & 2793.258 & 6145.1672 \\
\hline 6.777777778 & 281.629485 & 1126.518 & 2816.295 & 6195.8487 \\
\hline 6.805555556 & 283.942651 & 1135.771 & 2839.427 & 6246.7383 \\
\hline 6.833333333 & 286.265277 & 1145.061 & 2862.653 & 6297.8361 \\
\hline 6.861111111 & 288.597365 & 1154.389 & 2885.974 & 6349.142 \\
\hline 6.888888889 & 290.938913 & 1163.756 & 2909.389 & 6400. \\
\hline 6.9166 & 293.2 & 3.16 & 2.899 & 6452 \\
\hline 6.944444444 & 295.650391 & 1182.602 & 2956.504 & 6504.3086 \\
\hline 6.972222222 & 298.020321 & 1192.081 & 2980.203 & 6556.4471 \\
\hline 7 & 300.3 & 599 & 997 & 937 \\
\hline 7.027777778 & 302.788565 & 1211.154 & 3027.886 & 6661.3484 \\
\hline 7.055555556 & 305.186877 & 1220.748 & 3051.869 & 6714.1113 \\
\hline 7.083333333 & 307.5 & 379 & 947 & 676 \\
\hline 7.111111111 & 310.011885 & 1240.048 & 3100.119 & 6820 \\
\hline 7.138888889 & 312.438581 & 1249.754 & 3124.386 & 6873.6488 \\
\hline 7.1666666667 & 314.8 & 1259.499 & .747 & 692 \\
\hline 444444 & 317.3 & .281 & 204 & 6981. \\
\hline 7.222222222 & 319.775431 & 1279.102 & 3197.754 & 7035.0595 \\
\hline 7.25 & 322.2 & 1288.96 & 3222.4 & 7089.2793 \\
\hline 7.2777777778 & 324.7 & 1298.856 & 3247.14 & 7143.7073 \\
\hline 7.305555556 & 327.197429 & 1308.79 & 3271.974 & 7198.3434 \\
\hline 7.333333333 & 329.690349 & 1318.761 & 3296.903 & 7253.1877 \\
\hline 7.361111111 & 332.192731 & 1328.771 & 3321.927 & 7308.2401 \\
\hline 7.388888889 & 334.704573 & 1338.818 & 3347.046 & 7363.5006 \\
\hline 7.4166666667 & 337.225877 & 1348.904 & 3372.259 & 7418.9693 \\
\hline 7.444444444 & 339.756641 & 1359.027 & 3397.566 & 7474.6461 \\
\hline 7.472222222 & 342.296865 & 1369.187 & 3422.969 & 7530.531 \\
\hline 7.5 & 344.846551 & 1379.386 & 3448.466 & 7586.6241 \\
\hline 7.527777778 & 347.405697 & 1389.623 & 3474.057 & 7642.9253 \\
\hline 7.555555556 & 349.974305 & 1399.897 & 3499.743 & 7699.4347 \\
\hline 7.583333333 & 352.552373 & 1410.209 & 3525.524 & 7756.1522 \\
\hline 7.611111111 & 355.139901 & 1420.56 & 3551.399 & 7813.0778 \\
\hline 7.638888889 & 357.736891 & 1430.948 & 3577.369 & 7870.2116 \\
\hline 7.666666667 & 360.343341 & 1441.373 & 3603.433 & 7927.5535 \\
\hline 7.694444444 & 362.959253 & 1451.837 & 3629.593 & 7985.1036 \\
\hline 7.722222222 & 365.584625 & 1462.338 & 3655.846 & 8042.8617 \\
\hline 7.75 & 368.219457 & 1472.878 & 3682.195 & 8100.8281 \\
\hline 7.7777777778 & 370.863751 & 1483.455 & 3708.638 & 8159.0025 \\
\hline 7.805555556 & 373.517505 & 1494.07 & 3735.175 & 8217.3851 \\
\hline
\end{tabular}




\begin{tabular}{|c|c|c|c|c|}
\hline 7.833333333 & 376.180721 & 1504.723 & 3761.807 & 8275.9759 \\
\hline 7.861111111 & 378.853397 & 1515.414 & 3788.534 & 8334.7747 \\
\hline 7.888888889 & 381.535533 & 1526.142 & 3815.355 & 8393.7817 \\
\hline 7.916666667 & 384.227131 & 1536.909 & 3842.271 & 8452.9969 \\
\hline 7.944444444 & 386.928189 & 1547.713 & 3869.282 & 8512.4202 \\
\hline 7.972222222 & 389.638709 & 1558.555 & 3896.387 & 8572.0516 \\
\hline 8 & 392.358689 & 1569.435 & 3923.587 & 8631.8911 \\
\hline 8.027777778 & 395.088129 & 1580.353 & 3950.881 & 8691.9388 \\
\hline 8.055555556 & 397.827031 & 1591.308 & 3978.27 & 8752.1947 \\
\hline 8.083333333 & 400.575393 & 1602.302 & 4005.754 & 6587 \\
\hline 8.111111111 & 403.333217 & 1613.333 & 4033.332 & 8873.3308 \\
\hline 8.138888889 & 406.100501 & 1624.402 & 4061.005 & 8934.211 \\
\hline 8.166666667 & 408.877245 & 1635.509 & 4088.772 & 2994 \\
\hline 8.194444444 & 411.6 & 1646.654 & 4116.635 & 9056.5959 \\
\hline 8.222222222 & 414.459117 & 1657.836 & 4144.591 & 9118.1006 \\
\hline 8.25 & 417. & 1669.057 & 642 & 134 \\
\hline 8.277777778 & 420.078833 & 1680.315 & 4200.788 & 9241.7343 \\
\hline 8.305555556 & 422.902881 & 1691.612 & 4229.029 & 9303.8634 \\
\hline 8.333333333 & 425.736391 & 946 & 364 & 9366 \\
\hline 8.361111111 & 428.579361 & 1714.317 & 4285.794 & 9428.7459 \\
\hline 8.388888889 & 431.431793 & 1725.727 & 4314.318 & 9491.4994 \\
\hline 8.416666667 & 434.293685 & 1737.175 & 4342.937 & 611 \\
\hline 8.444444444 & 437.165037 & 1748.66 & 4371.65 & 9617.6308 \\
\hline 8.472222222 & 440.045851 & 1760.183 & 4400.459 & 9681.0087 \\
\hline 8.5 & 442.936125 & 1771.745 & 4429.361 & 5948 \\
\hline 8.527777778 & 445.8 & 1783.343 & 4458.359 & 3889 \\
\hline 8.555555556 & 448.745057 & 1794.98 & 4487.451 & 9872.3912 \\
\hline 8.583333333 & 451.663713 & 1806.655 & 4516.637 & 9936.6017 \\
\hline 8.611111111 & 454.591831 & 1818.367 & 4545.918 & 10001.02 \\
\hline 8.638888889 & 457.529409 & 1830.118 & 4575.294 & 10065.647 \\
\hline 8.666666667 & 460.476449 & 1841.906 & 4604.764 & 10130.482 \\
\hline 8.694444444 & 463.432949 & 1853.732 & 4634.329 & 10195.525 \\
\hline 8.722222222 & 466.398909 & 1865.596 & 4663.989 & 10260.776 \\
\hline 8.75 & 469.374331 & 1877.497 & 4693.743 & 10326.235 \\
\hline 8.777777778 & 472.359213 & 1889.437 & 4723.592 & 10391.903 \\
\hline 8.805555556 & 475.353557 & 1901.414 & 4753.536 & 10457.778 \\
\hline 8.833333333 & 478.357361 & 1913.429 & 4783.574 & 10523.862 \\
\hline 8.861111111 & 481.370625 & 1925.483 & 4813.706 & 10590.154 \\
\hline 8.888888889 & 484.393351 & 1937.573 & 4843.934 & 10656.654 \\
\hline 8.9166666667 & 487.425537 & 1949.702 & 4874.255 & 10723.362 \\
\hline 8.944444444 & 490.467185 & 1961.869 & 4904.672 & 10790.278 \\
\hline 8.972222222 & 493.518293 & 1974.073 & 4935.183 & 10857.402 \\
\hline 9 & 496.578861 & 1986.315 & 4965.789 & 10924.735 \\
\hline 9.027777778 & 499.648891 & 1998.596 & 4996.489 & 10992.276 \\
\hline 9.055555556 & 502.728381 & 2010.914 & 5027.284 & 11060.024 \\
\hline 9.083333333 & 505.817333 & 2023.269 & 5058.173 & 11127.981 \\
\hline
\end{tabular}




\begin{tabular}{|c|c|c|c|c|}
\hline 9.111111111 & 508.915745 & 2035.663 & 5089.157 & 11196.146 \\
\hline 9.138888889 & 512.023617 & 2048.094 & 5120.236 & 11264.52 \\
\hline 9.166666667 & 515.140951 & 2060.564 & 5151.41 & 11333.101 \\
\hline 9.194444444 & 518.267745 & 2073.071 & 5182.677 & 11401.89 \\
\hline 9.222222222 & 521.404001 & 2085.616 & 5214.04 & 11470.888 \\
\hline 9.25 & 524.549717 & 2098.199 & 5245.497 & 11540.094 \\
\hline 9.277777778 & 527.704893 & 2110.82 & 5277.049 & 11609.508 \\
\hline 9.305555556 & 530.869531 & 2123.478 & 5308.695 & 11679.13 \\
\hline 9.333333333 & 534.043629 & 2136.175 & 5340.436 & 11748.96 \\
\hline 9.361111111 & 537.227189 & 2148.909 & 5372.272 & 11818.998 \\
\hline 9.388888889 & 540.420209 & 2161.681 & 5404.202 & 11889.245 \\
\hline 9.416666667 & 543.622689 & 2174.491 & 5436.227 & 11959.699 \\
\hline 9.444444444 & 546.834631 & 2187.339 & 5468.346 & .362 \\
\hline 9.472222222 & 550.056033 & 2200.224 & 5500.56 & 12101.233 \\
\hline 9.5 & 553.286897 & 2213.148 & 5532.869 & 12172.312 \\
\hline 9.527777778 & 556.527221 & 2226.109 & & 43.599 \\
\hline 9.55 & 559. & 2239.108 & 5597.77 & 12315.094 \\
\hline 9.583333333 & 563.036251 & 2252.145 & 5630.363 & 12386.798 \\
\hline 9.611111111 & 566.304957 & 2265.22 & & .709 \\
\hline 9.638888889 & 569.58 & 2278.332 & 831 & 12530.829 \\
\hline 9.666666667 & 572.870753 & 2291.483 & 5728.708 & 12603.157 \\
\hline 9.694444444 & 576.167841 & 2304.671 & 5761.678 & 75.693 \\
\hline 22222 & 579.4 & .898 & .744 & 48.437 \\
\hline 9.75 & 582.790401 & .162 & 5827 & 1.389 \\
\hline 9.777777778 & 586.115873 & 2344.463 & 5861.159 & 12894.549 \\
\hline 9.805555556 & 589.4 & 803 & 508 & 7.918 \\
\hline 9.833333333 & 592.795197 & 2371.181 & 5927.952 & 13041.494 \\
\hline 9.861111111 & 596.149051 & 2384.596 & 5961.491 & 13115.279 \\
\hline 9.888888889 & 599.512365 & 2398.049 & .124 & 13189.272 \\
\hline 9.916666667 & 602.885141 & 2411.541 & 6028.851 & 13263.473 \\
\hline 9.944444444 & 606.267377 & 2425.07 & 6062.674 & 13337.882 \\
\hline 9.972222222 & 609.659073 & 2438.636 & 6096.591 & 13412.5 \\
\hline 10 & 613.060231 & 2452.241 & 6130.602 & 13487.325 \\
\hline 10.02777778 & 616.470849 & 2465.883 & 6164.708 & 13562.359 \\
\hline 555556 & 619.890929 & 2479.564 & 6198.909 & 13637.6 \\
\hline 10.08333333 & 623.320469 & 2493.282 & 6233.205 & 13713.05 \\
\hline 10.11111111 & 626.759469 & 2507.038 & 6267.595 & 13788.708 \\
\hline 10.13888889 & 630.207931 & 2520.832 & 6302.079 & 13864.574 \\
\hline 10.16666667 & 633.665853 & 2534.663 & 6336.659 & 13940.649 \\
\hline 10.19444444 & 637.133237 & 2548.533 & 6371.332 & 14016.931 \\
\hline 10.22222222 & 640.610081 & 2562.44 & 6406.101 & 14093.422 \\
\hline 10.25 & 644.096385 & 2576.386 & 6440.964 & 14170.12 \\
\hline 10.27777778 & 647.592151 & 2590.369 & 6475.922 & 14247.027 \\
\hline 10.30555556 & 651.097377 & 2604.39 & 6510.974 & 14324.142 \\
\hline 10.33333333 & 654.612065 & 2618.448 & 6546.121 & 14401.465 \\
\hline 10.36111111 & 658.136213 & 2632.545 & 6581.362 & 14478.997 \\
\hline
\end{tabular}




\begin{tabular}{|c|c|c|c|c|}
\hline 10.38888889 & 661.669821 & 2646.679 & 6616.698 & 14556.736 \\
\hline 10.41666667 & 665.212891 & 2660.852 & 6652.129 & 14634.684 \\
\hline 10.44444444 & 668.765421 & 2675.062 & 6687.654 & 14712.839 \\
\hline 10.47222222 & 672.327413 & 2689.31 & 6723.274 & 14791.203 \\
\hline 10.5 & 675.898865 & 2703.595 & 6758.989 & 14869.775 \\
\hline 10.52777778 & 679.479777 & 2717.919 & 6794.798 & 14948.555 \\
\hline 10.55555556 & 683.070151 & 2732.281 & 6830.702 & 15027.543 \\
\hline 10.58333333 & 686.669985 & 2746.68 & 6866.7 & 15106.74 \\
\hline 10.61111111 & 690.279281 & 2761.117 & 6902.793 & 15186.144 \\
\hline 10.63888889 & 693.898037 & 2775.592 & & 15265.757 \\
\hline 10.666666667 & 697.526253 & 2790.105 & 6975.263 & 15345.578 \\
\hline 10.69444444 & 701.163931 & 2804.656 & 7011.639 & 15425.606 \\
\hline 10.72222222 & 704.811 & 2819.244 & 7048.111 & 844 \\
\hline 10.75 & 708.4676 & 2833.871 & 7084.677 & 15586.289 \\
\hline 10.77777778 & 712.133729 & 2848.535 & 7121.337 & 15666.942 \\
\hline 10.80555556 & 715.80 & 2863.237 & 092 & 7.803 \\
\hline 10.83333333 & 719.494231 & 2877.977 & 7194.942 & 15828.873 \\
\hline 10.86111111 & 723.188673 & 2892.755 & 7231.887 & 15910.151 \\
\hline 10.88888889 & 726.85 & 2907.57 & 926 & 637 \\
\hline 10.91666667 & 730.6 & 2922.424 & .059 & .331 \\
\hline 10.94444444 & 734.328765 & 2937.315 & 7343.288 & 16155.233 \\
\hline 10.97222222 & 738.061051 & 2952.244 & 7380.611 & .343 \\
\hline 11 & 741.8 & .211 & 28 & .662 \\
\hline 11.02777778 & 745.55 & 2982.216 & 5.54 & 16402.188 \\
\hline 11.05555556 & 749.3 & 259 & 7493.147 & .923 \\
\hline 11.08333333 & 753.0 & .339 & 7530.848 & 7.866 \\
\hline 11.11111111 & 756.864391 & 3027.458 & 7568.644 & 16651.017 \\
\hline 11.13888889 & 760.653441 & 3042.614 & 7606.534 & 16734.376 \\
\hline 11.16666667 & 764.451953 & 3057.808 & 7644.52 & 16817.943 \\
\hline 11.19444444 & 768.259925 & 3073.04 & 7682.599 & 16901.718 \\
\hline 11.22222222 & 772.077357 & 3088.309 & 7720.774 & 16985.702 \\
\hline 11.25 & 775.904251 & 3103.617 & 7759.043 & 17069.894 \\
\hline 11.27777778 & 779.740605 & 3118.962 & 7797.406 & 17154.293 \\
\hline 11.30555556 & 783.586421 & 3134.346 & 7835.864 & 17238.901 \\
\hline 11.33333333 & 787.441697 & 3149.767 & 7874.417 & 17323.717 \\
\hline 11.36111111 & 791.306433 & 3165.226 & 7913.064 & 17408.742 \\
\hline 11.38888889 & 795.180631 & 3180.723 & 7951.806 & 17493.974 \\
\hline 11.41666667 & 799.064289 & 3196.257 & 7990.643 & 17579.414 \\
\hline 11.44444444 & 802.957409 & 3211.83 & 8029.574 & 17665.063 \\
\hline 11.47222222 & 806.859989 & 3227.44 & 8068.6 & 17750.92 \\
\hline 11.5 & 810.772029 & 3243.088 & 8107.72 & 17836.985 \\
\hline 11.52777778 & 814.693531 & 3258.774 & 8146.935 & 17923.258 \\
\hline 11.55555556 & 818.624493 & 3274.498 & 8186.245 & 18009.739 \\
\hline 11.58333333 & 822.564917 & 3290.26 & 8225.649 & 18096.428 \\
\hline 11.61111111 & 826.514801 & 3306.059 & 8265.148 & 18183.326 \\
\hline 11.63888889 & 830.474145 & 3321.897 & 8304.741 & 18270.431 \\
\hline
\end{tabular}

81 


\begin{tabular}{|c|c|c|c|c|}
\hline 11.66666667 & 834.442951 & 3337.772 & 8344.43 & 18357.745 \\
\hline 11.69444444 & 838.421217 & 3353.685 & 8384.212 & 18445.267 \\
\hline 11.72222222 & 842.408945 & 3369.636 & 8424.089 & 18532.997 \\
\hline 11.75 & 846.406133 & 3385.625 & 8464.061 & 18620.935 \\
\hline 11.77777778 & 850.412781 & 3401.651 & 8504.128 & 18709.081 \\
\hline 11.80555556 & 854.428891 & 3417.716 & 8544.289 & 18797.436 \\
\hline 11.83333333 & 858.454461 & 3433.818 & 8584.545 & 18885.998 \\
\hline 11.86111111 & 862.489493 & 3449.958 & 8624.895 & 18974.769 \\
\hline 11.88888889 & 866.533985 & 3466.136 & 8665.34 & 19063.748 \\
\hline 11.91666667 & 870.587937 & 3482.352 & 8705.879 & 2.935 \\
\hline 11.94444444 & 874.651351 & 3498.605 & 8746.514 & 19242.33 \\
\hline 11.97222222 & 878.724225 & 3514.897 & 8787.242 & 19331.933 \\
\hline 12 & 882.80 & 3531.226 & 8828.066 & .744 \\
\hline 12.02777778 & 886.898357 & 3547.593 & 8868.984 & 19511.764 \\
\hline 12.05555556 & 890.999613 & 3563.998 & 8909.996 & 19601.991 \\
\hline 12.08333333 & 895.11 & 3580 & 8951.103 & .427 \\
\hline 12.11111111 & 899.230509 & 3596.922 & 8992.305 & 19783.071 \\
\hline 12.13888889 & 903.360149 & 3613.441 & 9033.601 & 19873.923 \\
\hline 12.16666667 & 907.4 & 997 & 907 & .983 \\
\hline 12.19444444 & 911.6 & 3646.591 & 911 & 6.252 \\
\hline 12.22222222 & 915.805831 & 3663.223 & 9158.058 & 20147.728 \\
\hline 12.25 & 919.9 & 3679.893 & 9199.733 & .413 \\
\hline 12.27777778 & 924.15 & 3696.601 & 9241.503 & .306 \\
\hline 12.30555556 & 928.336661 & 3713.347 & 9283.367 & 20423.407 \\
\hline 12.33333333 & 932.532525 & 3730.13 & 9325.325 & 20515.716 \\
\hline 12.36111111 & 936.737851 & 3746.951 & 9367.379 & .233 \\
\hline 12.38888889 & 940.952637 & 3763.811 & 9409.526 & 20700.958 \\
\hline 12.41666667 & 945.176885 & 3780.708 & 9451.769 & 20793.891 \\
\hline 12.44444444 & 949.410593 & 3797.642 & 9494.106 & 20887.033 \\
\hline 12.47222222 & 953.653761 & 3814.615 & 9536.538 & 20980.383 \\
\hline 12.5 & 957.906391 & 3831.626 & 9579.064 & 21073.941 \\
\hline 12.52777778 & 962.168481 & 3848.674 & 9621.685 & 21167.707 \\
\hline 12.55555556 & 966.440033 & 3865.76 & 9664.4 & 21261.681 \\
\hline 12.58333333 & 970.721045 & 3882.884 & 9707.21 & 21355.863 \\
\hline 12.61111111 & 975.011517 & 3900.046 & 9750.115 & 21450.253 \\
\hline 12.63888889 & 979.311451 & 3917.246 & 9793.115 & 21544.852 \\
\hline 12.666666667 & 983.620845 & 3934.483 & 9836.208 & 21639.659 \\
\hline 12.69444444 & 987.939701 & 3951.759 & 9879.397 & 21734.673 \\
\hline 12.72222222 & 992.268017 & 3969.072 & 9922.68 & 21829.896 \\
\hline 12.75 & 996.605793 & 3986.423 & 9966.058 & 21925.327 \\
\hline 12.77777778 & 1000.95303 & 4003.812 & 10009.53 & 22020.967 \\
\hline 12.80555556 & 1005.30973 & 4021.239 & 10053.1 & 22116.814 \\
\hline 12.83333333 & 1009.67589 & 4038.704 & 10096.76 & 22212.87 \\
\hline 12.86111111 & 1014.05151 & 4056.206 & 10140.52 & 22309.133 \\
\hline 12.88888889 & 1018.43659 & 4073.746 & 10184.37 & 22405.605 \\
\hline 12.91666667 & 1022.83113 & 4091.325 & 10228.31 & 22502.285 \\
\hline
\end{tabular}




\begin{tabular}{|r|r|r|r|r|}
\hline 12.94444444 & 1027.23513 & 4108.941 & 10272.35 & 22599.173 \\
\hline 12.9722222 & 1031.6486 & 4126.594 & 10316.49 & 22696.269 \\
\hline 13 & 1036.07152 & 4144.286 & 10360.72 & 22793.573 \\
\hline 13.02777778 & 1040.50391 & 4162.016 & 10405.04 & 22891.086 \\
\hline 13.05555556 & 1044.94575 & 4179.783 & 10449.46 & 22988.807 \\
\hline 13.08333333 & 1049.39706 & 4197.588 & 10493.97 & 23086.735 \\
\hline 13.1111111 & 1053.85782 & 4215.431 & 10538.58 & 23184.872 \\
\hline 13.13888889 & 1058.32805 & 4233.312 & 10583.28 & 23283.217 \\
\hline 13.16666667 & 1062.80774 & 4251.231 & 10628.08 & 23381.77 \\
\hline 13.19444444 & 1067.29689 & 4269.188 & 10672.97 & 23480.532 \\
\hline 13.2222222 & 1071.7955 & 4287.182 & 10717.96 & 23579.501 \\
\hline 13.25 & 1076.30357 & 4305.214 & 10763.04 & 23678.679 \\
\hline 13.27777778 & 1080.8211 & 4323.284 & 10808.21 & 23778.064 \\
\hline 13.30555556 & 1085.3481 & 4341.392 & 10853.48 & 23877.658 \\
\hline 13.33333333 & 1089.88455 & 4359.538 & 10898.85 & 23977.46 \\
\hline 13.36111111 & 1094.43047 & 4377.722 & 10944.3 & 24077.47 \\
\hline 13.38888889 & 1098.98584 & 4395.943 & 10989.86 & 24177.688 \\
\hline 13.41666667 & 1103.55068 & 4414.203 & 11035.51 & 24278.115 \\
\hline 13.44444444 & 1108.12497 & 4432.5 & 11081.25 & 24378.749 \\
\hline 13.4722222 & 1112.70873 & 4450.835 & 11127.09 & 24479.592 \\
\hline 13.5 & 1117.30195 & 4469.208 & 11173.02 & 24580.643 \\
\hline 13.52777778 & 1121.90463 & 4487.619 & 11219.05 & 24681.902 \\
\hline 13.55555556 & 1126.51677 & 4506.067 & 11265.17 & 24783.369 \\
\hline 13.58333333 & 1131.13837 & 4524.553 & 11311.38 & 24885.044 \\
\hline 13.6111111 & 1135.76943 & 4543.078 & 11357.69 & 24986.927 \\
\hline 13.6388889 & 1140.40995 & 4561.64 & 11404.1 & 25089.019 \\
\hline 13.66666667 & 1145.05994 & 4580.24 & 11450.6 & 25191.319 \\
\hline 13.69444444 & 1149.71938 & 4598.878 & 11497.19 & 25293.826 \\
\hline 13.7222222 & 1154.38829 & 4617.553 & 11543.88 & 25396.542 \\
\hline 13.75 & 1159.06665 & 4636.267 & 11590.67 & 25499.466 \\
\hline 13.77777778 & 1163.75448 & 4655.018 & 11637.54 & 25602.599 \\
\hline 13.80555556 & 1168.45176 & 4673.807 & 11684.52 & 25705.939 \\
\hline 13.83333333 & 1173.15851 & 4692.634 & 11731.59 & 25809.487 \\
\hline 13.86111111 & 1177.87472 & 4711.499 & 11778.75 & 25913.244 \\
\hline 13.88888889 & 1182.60039 & 4730.402 & 11826 & 26017.209 \\
\hline & & & & \\
\hline
\end{tabular}

Figure F.3: Potential incident energy in equipment curve data

\begin{tabular}{|c|c|c|c|c|}
\hline \multirow{2}{*}{$\begin{array}{c}\text { Multiple of } \\
\text { Pickup }\end{array}$} & \multicolumn{4}{|c|}{ Incident Energy $\left(\mathrm{cal} / \mathrm{cm}^{2}\right)$} \\
\cline { 2 - 5 } & $\begin{array}{c}\text { Time Dial } \\
0.5\end{array}$ & $\begin{array}{c}\text { Time } \\
\text { Dial 2 }\end{array}$ & $\begin{array}{c}\text { Time } \\
\text { Dial 5 }\end{array}$ & $\begin{array}{c}\text { Time Dial } \\
11\end{array}$ \\
\hline 0.694444444 & 3.70106811 & 14.80427 & 37.01068 & 81.423498 \\
\hline 0.72222222 & 3.85853784 & 15.43415 & 38.58538 & 84.887833 \\
\hline 0.75 & 4.01638871 & 16.06555 & 40.16389 & 88.360552 \\
\hline 0.777777778 & 4.17460738 & 16.69843 & 41.74607 & 91.841362 \\
\hline 0.805555556 & 4.33318143 & 17.33273 & 43.33181 & 95.329991 \\
\hline 0.833333333 & 4.49209929 & 17.9684 & 44.92099 & 98.826184 \\
\hline
\end{tabular}

83 


\begin{tabular}{|c|c|c|c|c|}
\hline 0.861111111 & 4.65135015 & 18.6054 & 46.5135 & 102.3297 \\
\hline 0.888888889 & 4.81092387 & 19.2437 & 48.10924 & 105.84033 \\
\hline 0.9166666667 & 4.97081094 & 19.88324 & 49.70811 & 109.35784 \\
\hline 0.944444444 & 5.13100241 & 20.52401 & 51.31002 & 112.88205 \\
\hline 0.972222222 & 5.29148984 & 21.16596 & 52.9149 & 116.41278 \\
\hline 1 & 5.45226525 & 21.80906 & 54.52265 & 119.94984 \\
\hline 1.027777778 & 5.61332113 & 22.45328 & 56.13321 & 123.49306 \\
\hline 1.055555556 & 5.77465032 & 23.0986 & 57.7465 & 127.04231 \\
\hline 1.083333333 & 5.93624605 & 23.74498 & 59.36246 & 130.59741 \\
\hline 1.111111111 & 6.09810189 & 24.39241 & 60.98102 & 134.15824 \\
\hline 1.138888889 & 6.26021173 & 25.04085 & 62.60212 & 137.72466 \\
\hline 1.1666666667 & 6.42256973 & 25.69028 & 64.2257 & 141.2 \\
\hline 1.194444444 & 6.58517033 & 26.34068 & 65.8517 & 144.87375 \\
\hline 1.222222222 & 6.74800822 & 26.99203 & 67.48008 & 148.45618 \\
\hline 1.25 & 6.911078 & 27.64431 & 69.11078 & \\
\hline 1.277777778 & 7.07437583 & 28.2975 & 70.74376 & 155.63627 \\
\hline 1.305555556 & 7.23789604 & 28.95158 & 72.37896 & 159.23371 \\
\hline 1.333333333 & 7.40163 & 29.606 & 74.01635 & \\
\hline 1.361111111 & 7.565 & 30.26235 & 587 & 291 \\
\hline 1.388888889 & 7.72974934 & 30.919 & 9749 & 170. \\
\hline 1.4166666667 & 7.89411763 & 31.57647 & 78. & 7059 \\
\hline 1.444444444 & 8.058 & 475 & 80.5 & \\
\hline 1.472222222 & 8.223457 & 32.89383 & 3457 & 180.91605 \\
\hline 1.5 & 8.38842091 & 33.55368 & 83.88421 & 184.54526 \\
\hline 1.527777778 & & & & 7868 \\
\hline 1.555555556 & 8.71892019 & 34.87568 & 87.1892 & 191.81624 \\
\hline 1.583333333 & 8.88444913 & 35.5378 & 88.84449 & 195.45788 \\
\hline 1.611111111 & 9.05016017 & 36.20064 & 90.5016 & 0352 \\
\hline 1.638888889 & 9.21605036 & 36.8642 & 92.1605 & 202.75311 \\
\hline 1.6666666667 & 9.38211685 & 37.52847 & 93.82117 & 206.40657 \\
\hline 1.694444444 & 9.54835687 & & 95.48357 & 210.06385 \\
\hline 1.722222222 & 9.71476776 & 38.85907 & 4768 & 213.72489 \\
\hline 1.75 & 9.88134693 & 39.52539 & 98.81347 & 217.38963 \\
\hline 1.777777778 & 10.0480919 & 40.19237 & 100.4809 & 221.05802 \\
\hline 1.805555556 & 10.2150001 & 40.86 & 102.15 & 224.73 \\
\hline 1.833333333 & 10.3820694 & 41.52828 & 103.8207 & 228.40553 \\
\hline 1.861111111 & 10.5492973 & 42.19719 & 105.493 & 232.08454 \\
\hline 1.888888889 & 10.7166817 & 42.86673 & 107.1668 & 235.767 \\
\hline 1.9166666667 & 10.8842204 & 43.53688 & 108.8422 & 239.45285 \\
\hline 1.944444444 & 11.0519113 & 44.20765 & 110.5191 & 243.14205 \\
\hline 1.972222222 & 11.2197524 & 44.87901 & 112.1975 & 246.83455 \\
\hline 2 & 11.3877416 & 45.55097 & 113.8774 & 250.53032 \\
\hline 2.027777778 & 11.5558771 & 46.22351 & 115.5588 & 254.2293 \\
\hline 2.055555556 & 11.724157 & 46.89663 & 117.2416 & 257.93145 \\
\hline 2.083333333 & 11.8925793 & 47.57032 & 118.9258 & 261.63675 \\
\hline 2.111111111 & 12.0611425 & 48.24457 & 120.6114 & 265.34513 \\
\hline
\end{tabular}




\begin{tabular}{|c|c|c|c|c|}
\hline 2.138888889 & 12.2298446 & 48.91938 & 122.2984 & 269.05658 \\
\hline 2.1666666667 & 12.3986841 & 49.59474 & 123.9868 & 272.77105 \\
\hline 2.194444444 & 12.5676592 & 50.27064 & 125.6766 & 276.4885 \\
\hline 2.222222222 & 12.7367684 & 50.94707 & 127.3677 & 280.2089 \\
\hline 2.25 & 12.90601 & 51.62404 & 129.0601 & 283.93222 \\
\hline 2.277777778 & 13.0753826 & 52.30153 & 130.7538 & 287.65842 \\
\hline 2.305555556 & 13.2448847 & 52.97954 & 132.4488 & 291.38746 \\
\hline 2.333333333 & 13.4145147 & 53.65806 & 134.1451 & 295.11932 \\
\hline 2.361111111 & 13.5842713 & 54.33709 & 135.8427 & 298.85397 \\
\hline 2.388888889 & 13.754153 & 55.01661 & 5415 & 9137 \\
\hline 2.416666667 & 13.9241586 & 55.69663 & 139.2416 & 306.33149 \\
\hline 2.444444444 & 14.0942866 & 56.37715 & 140.9429 & 310.0743 \\
\hline 2.472222222 & 14.2645357 & 57.05814 & 142.6454 & 313.81979 \\
\hline 2.5 & 14.4 & 3962 & 144.349 & 317.5679 \\
\hline 2.527777778 & 14.6053923 & 58.42157 & 146.0539 & 321.31863 \\
\hline 2.555555556 & 14.7759974 & 399 & 7.76 & 325. \\
\hline 2.583333333 & 14.9467186 & 59.78687 & 149.4672 & 328.8 \\
\hline 2.611111111 & 15.1175549 & 60.47022 & 151.1755 & 332.58621 \\
\hline 2.6388 & 505 & 402 & 885 & 336 \\
\hline 2.6666666667 & 15.4595 & 827 & & 340 \\
\hline 2.694444444 & 15.6307424 & 62.52297 & 156.3074 & 343.87633 \\
\hline 2.722222222 & 15.8020276 & 63.20811 & 158.0203 & 347. \\
\hline 2.75 & & 63.89369 & & 529 \\
\hline 2.777777778 & 16.1449253 & 64.5797 & 493 & 355.18836 \\
\hline 2.805555556 & 16.3165359 & 65.26614 & 163.1654 & 358.96379 \\
\hline 2.833333333 & 16.488253 & 65.95301 & 164.8825 & 4157 \\
\hline 2.861111111 & 16.6600755 & 66.6403 & 166.6008 & 366.52166 \\
\hline 2.888888889 & 16.8320026 & 67.32801 & 168.32 & 370.30406 \\
\hline 2.916666667 & 17.0040332 & 68.01613 & 170.0403 & 374.08873 \\
\hline 2.944444444 & 17.1761665 & 68.70467 & 171.7617 & 377.87566 \\
\hline 2.972222222 & 17.3484015 & 69.39361 & 173.484 & 381.66483 \\
\hline 3 & 17.5207374 & 70.08295 & 175.2074 & 385.45622 \\
\hline 3.027777778 & 17.6931733 & 70.77269 & 176.9317 & 389.24981 \\
\hline 3.055555556 & 17.8657082 & 71.46283 & 178.6571 & 393.04558 \\
\hline 3.083333333 & 18.0383415 & 72.15337 & 180.3834 & 396.84351 \\
\hline 3.111111111 & 18.2110722 & 72.84429 & 182.1107 & 400.64359 \\
\hline 3.138888889 & 18.3838995 & 73.5356 & 183.839 & 404.44579 \\
\hline 3.166666667 & 18.5568227 & 74.22729 & 185.5682 & 408.2501 \\
\hline 3.194444444 & 18.7298409 & 74.91936 & 187.2984 & 412.0565 \\
\hline 3.222222222 & 18.9029533 & 75.61181 & 189.0295 & 415.86497 \\
\hline 3.25 & 19.0761592 & 76.30464 & 190.7616 & 419.6755 \\
\hline 3.277777778 & 19.2494579 & 76.99783 & 192.4946 & 423.48807 \\
\hline 3.305555556 & 19.4228486 & 77.69139 & 194.2285 & 427.30267 \\
\hline 3.333333333 & 19.5963306 & 78.38532 & 195.9633 & 431.11927 \\
\hline & 19.7699032 & 79.07961 & 197.699 & 434.93787 \\
\hline 3.388888889 & 19.9435656 & 79.77426 & 199.4357 & 438.75844 \\
\hline
\end{tabular}




\begin{tabular}{|c|c|c|c|c|}
\hline 3.416666667 & 20.1173172 & 80.46927 & 201.1732 & 442.58098 \\
\hline 3.444444444 & 20.2911573 & 81.16463 & 202.9116 & 446.40546 \\
\hline 3.472222222 & 20.4650852 & 81.86034 & 204.6509 & 450.23188 \\
\hline 3.5 & 20.6391003 & 82.5564 & 206.391 & 454.06021 \\
\hline 3.527777778 & 20.813202 & 83.25281 & 208.132 & 457.89044 \\
\hline 3.555555556 & 20.9873895 & 83.94956 & 209.8739 & 461.72257 \\
\hline 3.583333333 & 21.1616623 & 84.64665 & 211.6166 & 465.55657 \\
\hline 3.611111111 & 21.3360197 & 85.34408 & 213.3602 & 469.39243 \\
\hline 3.638888889 & 21.5104611 & 86.04184 & 215.1046 & 473.23014 \\
\hline 3.6666666667 & 21.6849859 & 86.73994 & 216.8499 & 477.06969 \\
\hline 3.694444444 & 21.8595936 & 87.43837 & 218.5959 & 480.91106 \\
\hline 3.722222222 & 22.0342835 & 88.13713 & 220.3428 & 484.75424 \\
\hline 3.75 & 22.2090551 & 88.83 & 222.0906 & 488.5 \\
\hline 3.777777778 & 22.3839078 & 89.5 & 223.8391 & 492.44597 \\
\hline 3.805555556 & 22.558841 & 90.23536 & 225.5884 & 496.2945 \\
\hline 3.833333333 & 22.7338542 & 90.93542 & 227. & 500.14479 \\
\hline 3.861111111 & 22.908 & 91.6 & 229.0895 & 503.99683 \\
\hline 3.888888889 & 23.0841185 & 92.33647 & 230.8412 & 507.85061 \\
\hline 3.9166666667 & 23.2593685 & 93.03747 & 937 & 611 \\
\hline 3.944444444 & 23.4346963 & & & 332 \\
\hline 3.972222222 & 23.6101015 & 94.44041 & 236.101 & 519.42223 \\
\hline 4 & 23.78 & 95.14233 & 237. & 8284 \\
\hline 4.0277777778 & 23.96 & 457 & & 4512 \\
\hline 4.055555556 & 24.136776 & 471 & 241.3 & 531.00907 \\
\hline 4.083333333 & 24.3124856 & 97.24994 & 243.1249 & 534.87468 \\
\hline 4.111111111 & 24.48827 & 97.95308 & 244.8827 & 538.74194 \\
\hline 4.138888889 & 24.6641289 & 98.65652 & 246.6413 & 542.61084 \\
\hline 4.1666666667 & 24.8400616 & 99.36025 & 248.4006 & 546.48136 \\
\hline 4.194444444 & 25.0160679 & 100.0643 & 250.1607 & 550.35349 \\
\hline 4.222222222 & 25.1921472 & 100.7686 & 251.9215 & 554.22724 \\
\hline 4.25 & 25.368299 & 101.4732 & 253.683 & 558.10258 \\
\hline 4.277777778 & 25.5445229 & 102.1781 & 255.4452 & 561.9795 \\
\hline 4.305555556 & 25.7208186 & 102.8833 & 257.2082 & 565.85801 \\
\hline 4.333333333 & 25.8971855 & 103.5887 & 258.9719 & 569.73808 \\
\hline 4.361111111 & 26.0736232 & 104.2945 & 260.7362 & 573.61971 \\
\hline 4.388888889 & 26.2501313 & 105.0005 & 262.5013 & 577.50289 \\
\hline 4.416666667 & 26.4267094 & 105.7068 & 264.2671 & 581.38761 \\
\hline 4.444444444 & 26.603357 & 106.4134 & 266.0336 & 585.27385 \\
\hline 4.472222222 & 26.7800738 & 107.1203 & 267.8007 & 589.16162 \\
\hline 4.5 & 26.9568594 & 107.8274 & 269.5686 & 593.05091 \\
\hline 4.527777778 & 27.1337133 & 108.5349 & 271.3371 & 596.94169 \\
\hline 4.555555556 & 27.3106352 & 109.2425 & 273.1064 & 600.83397 \\
\hline 4.583333333 & 27.4876246 & 109.9505 & 274.8762 & 604.72774 \\
\hline 4.611111111 & 27.6646813 & 110.6587 & 276.6468 & 608.62299 \\
\hline & 27.8418048 & & 278.418 & 612.5197 \\
\hline 4.6666666667 & 28.0189947 & 112.076 & 280.1899 & 616.41788 \\
\hline
\end{tabular}




\begin{tabular}{|c|c|c|c|c|}
\hline 4.694444444 & 28.1962506 & 112.785 & 281.9625 & 620.31751 \\
\hline 4.722222222 & 28.3735723 & 113.4943 & 283.7357 & 624.21859 \\
\hline 4.75 & 28.5509593 & 114.2038 & 285.5096 & 628.12111 \\
\hline 4.777777778 & 28.7284113 & 114.9136 & 287.2841 & 632.02505 \\
\hline 4.805555556 & 28.9059279 & 115.6237 & 289.0593 & 635.93041 \\
\hline 4.833333333 & 29.0835088 & 116.334 & 290.8351 & 639.83719 \\
\hline 4.861111111 & 29.2611536 & 117.0446 & 292.6115 & 643.74538 \\
\hline 4.888888889 & 29.438862 & 117.7554 & 294.3886 & 647.65497 \\
\hline 4.916666667 & 29.6166337 & 118.4665 & 296.1663 & 651.56594 \\
\hline 4.944444444 & 29.7944683 & 119.1779 & 297.9447 & 655.4783 \\
\hline 4.972222222 & 29.9723654 & 119.8895 & 299.7237 & 659.39204 \\
\hline 5 & 30.1503248 & 120.6013 & 301.5032 & 663.30715 \\
\hline 5.027777778 & 30.328 & & 2835 & 2361 \\
\hline 5.055555556 & 30.5064291 & 122.0257 & 305.0643 & 671.14144 \\
\hline 5.083333333 & 30.6845733 & 122.7383 & 306.8457 & 675.06061 \\
\hline 5.111111111 & & & 278 & 113 \\
\hline 5.138888889 & 31.0410444 & 124. & 310.4104 & 682.90298 \\
\hline 5.166666667 & 31.2193706 & 124.8775 & 312.1937 & 686.82615 \\
\hline 5.194444444 & & & 776 & 065 \\
\hline 22222 & 31.57 & 126.3048 & 5.762 & 694.67646 \\
\hline 5.25 & 31.7547084 & 127.0188 & 317.5471 & 698.60358 \\
\hline 5.277777778 & 273 & 331 & 319.3327 & 3201 \\
\hline 5.305555556 & 32.111 & 4476 & 321.119 & 706.46172 \\
\hline 5.333333333 & 32.2905786 & 623 & 322.9058 & 710.39273 \\
\hline 5.361111111 & 32.469319 & 129.8773 & 324.6932 & 714.32502 \\
\hline 5.388888889 & 32.6481174 & 130.5925 & 326.4812 & 718.25858 \\
\hline 5.416666667 & 32.8269735 & 131.3079 & 328.2697 & 722.19342 \\
\hline 5.444444444 & 33.0058871 & 132.0235 & 330.0589 & 726.12952 \\
\hline 5.472222222 & 33.184 & 394 & 331.8486 & 6687 \\
\hline 5.5 & 33.3638855 & 133.4 & 333.6389 & 734.00548 \\
\hline 5.527777778 & 33.5429698 & 134.1719 & 335.4297 & 737.94534 \\
\hline 5.555555556 & 33.7221104 & 134.8884 & 337.2211 & 741.88643 \\
\hline 5.583333333 & 33.9013072 & 135.6052 & 339.0131 & 745.82876 \\
\hline 5.611111111 & 34.0805598 & 136.3222 & 340.8056 & 749.77232 \\
\hline 5.638888889 & 34.259868 & 137.0395 & 342.5987 & 753.7171 \\
\hline 5.666666667 & 34.4392315 & 137.7569 & 344.3923 & 757.66309 \\
\hline 5.694444444 & 34.6186501 & 138.4746 & 346.1865 & 761.6103 \\
\hline 5.722222222 & 34.7981235 & 139.1925 & 347.9812 & 765.55872 \\
\hline 5.75 & 34.9776515 & 139.9106 & 349.7765 & 769.50833 \\
\hline 5.777777778 & 35.1572338 & 140.6289 & 351.5723 & 773.45914 \\
\hline 5.805555556 & 35.3368702 & 141.3475 & 353.3687 & 777.41114 \\
\hline 5.833333333 & 35.5165604 & 142.0662 & 355.1656 & 781.36433 \\
\hline 5.861111111 & 35.6963042 & 142.7852 & 356.963 & 785.31869 \\
\hline 5.888888889 & 35.8761014 & 143.5044 & 358.761 & 789.27423 \\
\hline 5.916666667 & 36.0559516 & 144.2238 & 360.5595 & 793.23094 \\
\hline 5.944444444 & 36.2358548 & 144.9434 & 362.3585 & 797.18881 \\
\hline
\end{tabular}




\begin{tabular}{|c|c|c|c|c|}
\hline 5.972222222 & 36.4158106 & 145.6632 & 364.1581 & 801.14783 \\
\hline 6 & 36.5958189 & 146.3833 & 365.9582 & 805.10802 \\
\hline 6.027777778 & 36.7758794 & 147.1035 & 367.7588 & 809.06935 \\
\hline 6.055555556 & 36.9559918 & 147.824 & 369.5599 & 813.03182 \\
\hline 6.083333333 & 37.136156 & 148.5446 & 371.3616 & 816.99543 \\
\hline 6.111111111 & 37.3163717 & 149.2655 & 373.1637 & 820.96018 \\
\hline 6.138888889 & 37.4966387 & 149.9866 & 374.9664 & 824.9 \\
\hline 6.1666666667 & 37.6769568 & 150.7078 & 376.7696 & 828.89305 \\
\hline 6.194444444 & 37.8573258 & 151.4293 & 378.5733 & 832.86117 \\
\hline 6.222222222 & 38.0377454 & 152.151 & 380.3775 & 836.8304 \\
\hline 6.25 & 38.2182155 & 152.8729 & 382.1822 & 840.80074 \\
\hline 6.277777778 & 38.3987358 & 153.5949 & 383.9874 & 844.77219 \\
\hline 6.305555556 & 38.5793062 & 154.3172 & 385.7931 & 848. \\
\hline 6.33 & 38.7 & & 5993 & 838 \\
\hline 6.361111111 & 38.9405962 & 155.7624 & 389.406 & 856.69312 \\
\hline 6.388888889 & 39.1213154 & 156.4853 & 391.2132 & 860.66894 \\
\hline 6.4166 & 39.3 & & 208 & \\
\hline 6.444444444 & 39.482 & 316 & .829 & 868.62383 \\
\hline 6.472222222 & 39.6637675 & 158.6551 & 396.6377 & 872.60289 \\
\hline 6.5 & 39.84 & 787 & 4468 & 301 \\
\hline 6.527777778 & 40.0256457 & 160.1026 & 400.2565 & 880.56421 \\
\hline 6.555555556 & 40.2066573 & 160.8266 & 402.0666 & 884.54646 \\
\hline 6.583333333 & 40.38 & 509 & 8772 & 2977 \\
\hline 6.611111111 & 40.5688243 & 2753 & 405.6882 & 892.51413 \\
\hline 6.638888889 & 40.7499794 & 999 & 407.4998 & 896.49955 \\
\hline 6.6666666667 & 40.9311819 & 163.7247 & 409.3118 & 900.486 \\
\hline 6.694444444 & 41.1124318 & 164.4497 & 411.1243 & 904.4735 \\
\hline 6.722222222 & 41.2937287 & 165.1749 & 412.9373 & 908.46203 \\
\hline 6.75 & 41.4750726 & 165.9003 & 414.7507 & 912.4516 \\
\hline 6.7777777778 & 41.6564633 & 166.6259 & 416.5646 & 916.44219 \\
\hline 6.805555556 & 41.8379004 & 167.3516 & 418.379 & 920.43381 \\
\hline 6.833333333 & 42.019384 & 168.0775 & 420.1938 & 924.42645 \\
\hline 6.861111111 & 42.2009138 & 168.8037 & 422.0091 & 928.4201 \\
\hline 6.888888889 & 42.3824896 & 169.53 & 423.8249 & 932.41477 \\
\hline 6.9166666667 & 42.5641113 & 170.2564 & 425.6411 & 936.41045 \\
\hline 6.944444444 & 42.7457786 & 170.9831 & 427.4578 & 940.40713 \\
\hline 6.972222222 & 42.9274915 & 171.71 & 429.2749 & 944.40481 \\
\hline 7 & 43.1092497 & 172.437 & 431.0925 & 948.40349 \\
\hline 7.027777778 & 43.2910531 & 173.1642 & 432.9105 & 952.40317 \\
\hline 7.055555556 & 43.4729015 & 173.8916 & 434.729 & 956.40383 \\
\hline 7.083333333 & 43.6547947 & 174.6192 & 436.5479 & 960.40548 \\
\hline 7.111111111 & 43.8367326 & 175.3469 & 438.3673 & 964.40812 \\
\hline 7.138888889 & 44.018715 & 176.0749 & 440.1872 & 968.41173 \\
\hline 7.166666667 & 44.2007418 & 176.803 & 442.0074 & 972.41632 \\
\hline 7.194444444 & 44.3828128 & 177.5313 & 443.8281 & 976.42188 \\
\hline 7.222222222 & 44.5649278 & 178.2597 & 445.6493 & 980.42841 \\
\hline
\end{tabular}




\begin{tabular}{|c|c|c|c|c|}
\hline 7.25 & 44.7470866 & 178.9883 & 447.4709 & 984.43591 \\
\hline 7.277777778 & 44.9292892 & 179.7172 & 449.2929 & 988.44436 \\
\hline 7.305555556 & 45.1115354 & 180.4461 & 451.1154 & 992.45378 \\
\hline 7.333333333 & 45.2938249 & 181.1753 & 452.9382 & 996.46415 \\
\hline 7.361111111 & 45.4761577 & 181.9046 & 454.7616 & 1000.4755 \\
\hline 7.388888889 & 45.6585335 & 182.6341 & 456.5853 & 1004.4877 \\
\hline 7.416666667 & 45.8409524 & 183.3638 & 458.4095 & 1008.501 \\
\hline 7.444444444 & 46.023414 & 184.0937 & 460.2341 & 1012.5151 \\
\hline 7.472222222 & 46.2059182 & 184.8237 & 462.0592 & 1016.5302 \\
\hline 7.5 & 46.388465 & 185.5539 & 463.8846 & .5462 \\
\hline 7.527777778 & 46.5710541 & 186.2842 & 465.7105 & 1024.5632 \\
\hline 7.555555556 & 46.7536854 & 187.0147 & 467.5369 & 1028.5811 \\
\hline 7.583333333 & 46.9363587 & 187.7454 & 469. & \\
\hline 7.611111111 & 47.119074 & 188.4763 & 471.1907 & 1036.6196 \\
\hline 7.638888889 & 47.301831 & 189.2073 & 473.0183 & 1040.6403 \\
\hline 7.6666666667 & 47.4846296 & 189.9385 & 474.8 & 1044 \\
\hline 7.694444444 & 47.6674697 & 190.6 & 476.6747 & 1048.6843 \\
\hline 7.722222222 & 47.8503512 & 191.4014 & 478.5035 & 1052.7077 \\
\hline 7.75 & 48.0332739 & 192.1 & 480. & 1056 \\
\hline 7.777777778 & 48.2 & .865 & 482 & \\
\hline 7.805555556 & 48.3992423 & 193.597 & 483.9924 & 1064.7833 \\
\hline 7.833333333 & 48.5822877 & 194.3292 & 485.8229 & 103 \\
\hline 7.861111111 & 48.76 & 195.0615 & 487. & \\
\hline 7.888888889 & 48.9485004 & 195.794 & 489.485 & 1076.867 \\
\hline 7.916666667 & 49.1316675 & 196.5267 & 491.3167 & 1080.8967 \\
\hline 7.944444444 & 49.3148747 & & 493.1487 & \\
\hline 7.972222222 & 49.4981221 & 197.9925 & 494.9812 & 1088.9587 \\
\hline 8 & 49.6814095 & 198.7256 & 496.8141 & 1092.991 \\
\hline 8.027777778 & 49.8647367 & 199.4589 & 498.6474 & 1097.0242 \\
\hline 8.055555556 & 50.0481037 & 200.1924 & 500.481 & 1101.0583 \\
\hline 8.083333333 & 50.2315102 & 200.926 & 502.3151 & 1105.0932 \\
\hline 8.111111111 & 50.4149563 & 201.6598 & 504.1496 & 1109.129 \\
\hline 8.138888889 & 50.5984417 & 202.3938 & 505.9844 & 1113.1657 \\
\hline 8.166666667 & 50.7819663 & 203.1279 & 507.8197 & 1117.2033 \\
\hline 8.194444444 & 50.96553 & 203.8621 & 509.6553 & 1121.2417 \\
\hline 8.222222222 & 51.1491327 & 204.5965 & 511.4913 & 1125.2809 \\
\hline 8.25 & 51.3327742 & 205.3311 & 513.3277 & 1129.321 \\
\hline 8.277777778 & 51.5164544 & 206.0658 & 515.1645 & 1133.362 \\
\hline 8.305555556 & 51.7001733 & 206.8007 & 517.0017 & 1137.4038 \\
\hline 8.333333333 & 51.8839306 & 207.5357 & 518.8393 & 1141.4465 \\
\hline 8.361111111 & 52.0677263 & 208.2709 & 520.6773 & 1145.49 \\
\hline 8.388888889 & 52.2515603 & 209.0062 & 522.5156 & 1149.5343 \\
\hline 8.416666667 & 52.4354324 & 209.7417 & 524.3543 & 1153.5795 \\
\hline 8.444444444 & 52.6193424 & 210.4774 & 526.1934 & 1157.6255 \\
\hline 8.472222222 & 52.8032904 & 211.2132 & 528.0329 & 1161.6724 \\
\hline 8.5 & 52.9872761 & 211.9491 & 529.8728 & 1165.7201 \\
\hline
\end{tabular}




\begin{tabular}{|c|c|c|c|c|}
\hline 8.527777778 & 53.1712995 & 212.6852 & 531.713 & 1169.7686 \\
\hline 8.555555556 & 53.3553605 & 213.4214 & 533.5536 & 1173.8179 \\
\hline 8.583333333 & 53.5394589 & 214.1578 & 535.3946 & 1177.8681 \\
\hline 8.611111111 & 53.7235945 & 214.8944 & 537.2359 & 1181.9191 \\
\hline 8.638888889 & 53.9077674 & 215.6311 & 539.0777 & 1185.9709 \\
\hline 8.666666667 & 54.0919774 & 216.3679 & 540.9198 & 1190.0235 \\
\hline 8.694444444 & 54.2762244 & 217.1049 & 542.7622 & 1194.0769 \\
\hline 8.722222222 & 54.4605082 & 217.842 & 544.6051 & 1198.1312 \\
\hline 8.75 & 54.6448288 & 218.5793 & 546.4483 & 1202.1862 \\
\hline 8.777777778 & 54.829186 & 219.3167 & 548.2919 & 1206.2421 \\
\hline 8.805555556 & 55.0135798 & 220.0 & 550.1358 & 10.2988 \\
\hline 8.833333333 & 55.19801 & 220.792 & 551.9801 & 1214.3562 \\
\hline 8.861111111 & 55.3824765 & 221.5299 & 553. & 145 \\
\hline 8.888888889 & 55.56 & 222.2679 & 555.6698 & 1222.4735 \\
\hline 8.916666667 & 55.7515181 & 223.0061 & 557.5152 & 1226.5334 \\
\hline 8.944444444 & 55.93 & & 559. & 1230.594 \\
\hline 8.972222222 & 56.1207037 & 224.4828 & 561.207 & 1234.6555 \\
\hline 9 & 56.3053503 & 225.2214 & 563.0535 & 1238.7177 \\
\hline 9.027777778 & 56.49 & 225.9 & 564. & 807 \\
\hline 5556 & 56.672 & & 566.7 & 46.8445 \\
\hline 9.083333333 & 56.8595037 & 227. & 568.595 & 1250.9091 \\
\hline 9.111111111 & 57.0442924 & 228.1772 & 570.4429 & 254.9744 \\
\hline 9.138888889 & 57.2291164 & & 572. & 259.0406 \\
\hline 9.1666666667 & 57.4139756 & 229.6 & 574.1398 & 1263.1075 \\
\hline 9.194444444 & 57.5988698 & 230.3 & 575.9 & 1267.1751 \\
\hline 9.222222222 & 57.7837991 & & 838 & 271.2436 \\
\hline 9.25 & 57.9687632 & 231.8751 & 579.6 & 1275.3128 \\
\hline 9.277777778 & 58.1537621 & 232.615 & 581.5376 & 1279.3828 \\
\hline 9.305555556 & 58.3387957 & 233.3552 & 583.388 & 1283.4535 \\
\hline 9.333333333 & 58.5238639 & 234.0955 & 585.2386 & 1287.525 \\
\hline 9.361111111 & 58.7089667 & 234.8359 & 587.0897 & 1291.5973 \\
\hline 9.388888889 & 58.8941038 & 235.5764 & 588.941 & 1295.6703 \\
\hline 9.4166666667 & 59.0792752 & 236.3171 & 590.7928 & 1299.7441 \\
\hline 9.444444444 & 59.2644808 & 237.0579 & 592.6448 & 1303.8186 \\
\hline 9.472222222 & 59.4497205 & 237.7989 & 594.4972 & 1307.8939 \\
\hline 9.5 & 59.6349943 & 238.54 & 596.3499 & 1311.9699 \\
\hline 9.527777778 & 59.820302 & 239.2812 & 598.203 & 1316.0466 \\
\hline 9.555555556 & 60.0056435 & 240.0226 & 600.0564 & 1320.1242 \\
\hline 9.583333333 & 60.1910188 & 240.7641 & 601.9102 & 1324.2024 \\
\hline 9.611111111 & 60.3764277 & 241.5057 & 603.7643 & 1328.2814 \\
\hline 9.638888889 & 60.5618702 & 242.2475 & 605.6187 & 1332.3611 \\
\hline 9.6666666667 & 60.7473461 & 242.9894 & 607.4735 & 1336.4416 \\
\hline 9.694444444 & 60.9328554 & 243.7314 & 609.3286 & 1340.5228 \\
\hline 9.722222222 & 61.1183981 & 244.4736 & 611.184 & 1344.6048 \\
\hline 9.75 & 61.3039739 & 245.2159 & 613.0397 & 1348.6874 \\
\hline 9.777777778 & 61.4895828 & 245.9583 & 614.8958 & 1352.7708 \\
\hline
\end{tabular}




\begin{tabular}{|c|c|c|c|c|}
\hline 9.805555556 & 61.6752248 & 246.7009 & 616.7522 & 1356.8549 \\
\hline 9.833333333 & 61.8608997 & 247.4436 & 618.609 & 1360.9398 \\
\hline 9.861111111 & 62.0466074 & 248.1864 & 620.4661 & 1365.0254 \\
\hline 9.888888889 & 62.2323479 & 248.9294 & 622.3235 & 1369.1117 \\
\hline 9.916666667 & 62.4181211 & 249.6725 & 624.1812 & 1373.1987 \\
\hline 9.944444444 & 62.6039269 & 250.4157 & 626.0393 & 1377.2864 \\
\hline 9.972222222 & 62.7897652 & 251.1591 & 627.8977 & 1381.3748 \\
\hline 10 & 62.9756359 & 251.9025 & 629.7564 & 1385.464 \\
\hline 10.02777778 & 63.1615389 & 252.6462 & 631.6154 & 1389.5539 \\
\hline 10.05555556 & 63.3474742 & 253.3899 & 633.4747 & 1393.6444 \\
\hline 10.08333333 & 63.5334417 & 254.1338 & 635.3344 & 1397.7357 \\
\hline 10.11111111 & 63.7194412 & 254.8778 & 637.1944 & 1401.8277 \\
\hline 10.13888889 & 63.90 & 255. & 639 & 204 \\
\hline 10.16666667 & 64.05 & 256.3661 & 640.9154 & 1410.0138 \\
\hline 10.19444444 & 64.2776315 & 257.1105 & 642.7763 & 1414.1079 \\
\hline 10.22222222 & 64.46 & 257.855 & 644 & \\
\hline 10.25 & 64.6499174 & 258.5997 & 646.4992 & \\
\hline 10.27777778 & 64.8361077 & 259.3444 & 648.3611 & 1426.3944 \\
\hline 10.30555556 & 65.02 & 260. & 650 & \\
\hline 10.33333333 & & 343 & 652. & \\
\hline 10.36111111 & 65.3948675 & 261.5795 & 653.9487 & 1438.6871 \\
\hline 10.38888889 & 65.5811834 & 262.3247 & 655. & 786 \\
\hline 10.41666667 & 65.7 & 701 & 657 & 1446.8857 \\
\hline 10.44444444 & 65.9539088 & 263.8156 & 659. & 1450.986 \\
\hline 10.47222222 & 66.1403181 & 5613 & 661 & 087 \\
\hline 10.5 & 66.3 & 307 & 676 & 1459.1887 \\
\hline 10.52777778 & 66.5132295 & 266.0529 & 665.1323 & 1463.291 \\
\hline 10.55555556 & 66.6997315 & 266.7989 & 666.9973 & 1467.3941 \\
\hline 10.58333333 & 66.8862642 & 267.5451 & 668.8626 & 1471.4978 \\
\hline 10.61111111 & 67.0728275 & 268.2913 & 670.7283 & 1475.6022 \\
\hline 10.63888889 & 67.2594215 & 269.0377 & 672.5942 & 1479.7073 \\
\hline 10.66666667 & 67.446046 & 269.7842 & 674.4605 & 1483.813 \\
\hline 10.69444444 & 67.6327009 & 270.5308 & 676.327 & 1487.9194 \\
\hline 10.72222222 & 67.8193861 & 271.2775 & 678.1939 & 1492.0265 \\
\hline 10.75 & 68.0061017 & 272.0244 & 680.061 & 1496.1342 \\
\hline 10.77777778 & 68.1928475 & 272.7714 & 681.9285 & 1500.2426 \\
\hline 10.80555556 & 68.3796234 & 273.5185 & 683.7962 & 1504.3517 \\
\hline 10.83333333 & 68.5664294 & 274.2657 & 685.6643 & 1508.4614 \\
\hline 10.86111111 & 68.7532654 & 275.0131 & 687.5327 & 1512.5718 \\
\hline 10.88888889 & 68.9401313 & 275.7605 & 689.4013 & 1516.6829 \\
\hline 10.916666667 & 69.1270271 & 276.5081 & 691.2703 & 1520.7946 \\
\hline 10.94444444 & 69.3139526 & 277.2558 & 693.1395 & 1524.907 \\
\hline 10.97222222 & 69.5009079 & 278.0036 & 695.0091 & 1529.02 \\
\hline 11 & 69.6878928 & 278.7516 & 696.8789 & 1533.1336 \\
\hline 11.02777778 & 69.8749073 & 279.4996 & 698.7491 & 1537.248 \\
\hline 11.05555556 & 70.0619513 & 280.2478 & 700.6195 & 1541.3629 \\
\hline
\end{tabular}

91 


\begin{tabular}{|c|c|c|c|c|}
\hline 11.08333333 & 70.2490247 & 280.9961 & 702.4902 & 1545.4785 \\
\hline 11.11111111 & 70.4361275 & 281.7445 & 704.3613 & 1549.5948 \\
\hline 11.13888889 & 70.6232595 & 282.493 & 706.2326 & 1553.7117 \\
\hline 11.16666667 & 70.8104208 & 283.2417 & 708.1042 & 1557.8293 \\
\hline 11.19444444 & 70.9976113 & 283.9904 & 709.9761 & 1561.9474 \\
\hline 11.22222222 & 71.1848308 & 284.7393 & 711.8483 & 1566.0663 \\
\hline 11.25 & 71.3720794 & 285.4883 & 713.7208 & 1570.1857 \\
\hline 11.27777778 & 71.559357 & 286.2374 & 715.5936 & 1574.3059 \\
\hline 11.30555556 & 71.7466634 & 286.9867 & 717.4666 & 1578.4266 \\
\hline 11.33333333 & 71.9339986 & 287.736 & 719.34 & \\
\hline 11.36111111 & 72.1213626 & 288.4855 & 721.2136 & 1586.67 \\
\hline 11.38888889 & 72.3087553 & 289.235 & 723.0876 & 1590.7926 \\
\hline & 72.49 & 289.9847 & 724.9618 & \\
\hline 11.44444444 & 72.6836265 & 290.7345 & 726.8363 & 1599.0398 \\
\hline 11.47222222 & 72.8711049 & 291.4844 & 728.711 & 1603.1643 \\
\hline 11.5 & 73.0586117 & 292.2344 & 730.5861 & 895 \\
\hline 11.52777778 & 73.2461468 & 292.9846 & 732.4615 & 1611.4152 \\
\hline 11.55555556 & 73.4337103 & 293.7348 & 734.3371 & 1615.5416 \\
\hline 11.58333333 & 73.6 & 294.4852 & 213 & 686 \\
\hline 11.61111111 & 73.8089219 & 295.2357 & 738.0892 & \\
\hline 11.63888889 & 73.9965699 & 295.9863 & 739.9657 & 1627.9245 \\
\hline 11.66666667 & 74.1842459 & 296.737 & 741.8425 & 1632.0534 \\
\hline 11.69444444 & 74.3719499 & 297.4878 & 195 & 1636.1829 \\
\hline 11.72222222 & 74.5596819 & 298.2387 & 745.5968 & 1640.313 \\
\hline 11.75 & 74.7474417 & 298.9898 & 747.4744 & 1644.4437 \\
\hline 11.77777778 & 74.93 & 299.7409 & 749.3523 & 1648.575 \\
\hline 11.80555556 & 75.1230446 & 300.4922 & 751.2304 & 1652.707 \\
\hline 11.83333333 & 75.3108876 & 301.2436 & 753.1089 & 1656.8395 \\
\hline 11.86111111 & 75.4987583 & 301.995 & 754.9876 & 1660.9727 \\
\hline 11.88888889 & 75.6866565 & 302.7466 & 756.8666 & 1665.1064 \\
\hline 11.91666667 & 75.8745821 & 303.4983 & 758.7458 & 1669.2408 \\
\hline 11.94444444 & 76.0625353 & 304.2501 & 760.6254 & 1673.3758 \\
\hline 11.97222222 & 76.2505158 & 305.0021 & 762.5052 & 1677.5113 \\
\hline 12 & 76.4385236 & 305.7541 & 764.3852 & 1681.6475 \\
\hline 12.02777778 & 76.6265586 & 306.5062 & 766.2656 & 1685.7843 \\
\hline 12.05555556 & 76.8146209 & 307.2585 & 768.1462 & 1689.9217 \\
\hline 12.08333333 & 77.0027103 & 308.0108 & 770.0271 & 1694.0596 \\
\hline 12.11111111 & 77.1908268 & 308.7633 & 771.9083 & 1698.1982 \\
\hline 12.13888889 & 77.3789703 & 309.5159 & 773.7897 & 1702.3373 \\
\hline 12.16666667 & 77.5671408 & 310.2686 & 775.6714 & 1706.4771 \\
\hline 12.19444444 & 77.7553381 & 311.0214 & 777.5534 & 1710.6174 \\
\hline 12.22222222 & 77.9435623 & 311.7742 & 779.4356 & 1714.7584 \\
\hline 12.25 & 78.1318134 & 312.5273 & 781.3181 & 1718.8999 \\
\hline 12.27777778 & 78.3200911 & 313.2804 & 783.2009 & 1723.042 \\
\hline & 78.5083955 & & 785.084 & 1727.1847 \\
\hline 12.33333333 & 78.6967266 & 314.7869 & 786.9673 & 1731.328 \\
\hline
\end{tabular}




\begin{tabular}{|c|c|c|c|c|}
\hline 12.36111111 & 78.8850842 & 315.5403 & 788.8508 & 1735.4719 \\
\hline 12.38888889 & 79.0734683 & 316.2939 & 790.7347 & 1739.6163 \\
\hline 12.41666667 & 79.2618789 & 317.0475 & 792.6188 & 1743.7613 \\
\hline 12.44444444 & 79.4503158 & 317.8013 & 794.5032 & 1747.9069 \\
\hline 12.47222222 & 79.6387792 & 318.5551 & 796.3878 & 1752.0531 \\
\hline 12.5 & 79.8272688 & 319.3091 & 798.2727 & 1756.1999 \\
\hline 12.52777778 & 80.0157846 & 320.0631 & 800.1578 & 1760.3473 \\
\hline 12.55555556 & 80.2043266 & 320.8173 & 802.0433 & 1764.4952 \\
\hline 12.58333333 & 80.3928947 & 321.5716 & 803.9289 & 1768.6437 \\
\hline 12.61111111 & 80.5814889 & 322.326 & 805.8149 & 1772.7928 \\
\hline 12.63888889 & 80.7701092 & 323.0804 & 807.7011 & 1776.9424 \\
\hline 12.66666667 & 80.9587554 & 323.835 & 809.5876 & 1781.0926 \\
\hline 12.69444444 & 81.1474274 & 8997 & 811.4743 & 434 \\
\hline 12.72222222 & 81.3361254 & 325.3445 & 813.3613 & 1789.3948 \\
\hline 12.75 & 81.5248491 & 326.0994 & 815.2485 & 1793.5467 \\
\hline 12.77777778 & 81.7135986 & 544 & & 1797.6992 \\
\hline 12.80555556 & 81.9023738 & 327.6095 & 819.0237 & 1801.8522 \\
\hline 12.83333333 & 82.0911747 & 328.3647 & 820.9117 & 1806.0058 \\
\hline 12.86111111 & 82.280 & 329.12 & 822.8 & \\
\hline 12.88888889 & 82.4 & 329.8754 & 824.6885 & 1814.3148 \\
\hline 12.91666667 & 82.6577306 & 330.6309 & 826.5773 & 1818.4701 \\
\hline 12.94444444 & 82.8466335 & 331.3865 & 828.4663 & 1822.6259 \\
\hline 12.97222222 & 83.0 & 422 & 556 & 824 \\
\hline 13 & 83.2245154 & 332.8981 & 832.2452 & 1830.9393 \\
\hline 13.02777778 & 83.4134943 & 333.654 & 834.1349 & 1835.0969 \\
\hline 13.05555556 & 83.60 & 334.41 & 36.025 & .255 \\
\hline 13.08333333 & 83.7915278 & 335.1661 & 837.9153 & 1843.4136 \\
\hline 13.11111111 & 83.9805823 & 335.9223 & 839.8058 & 1847.5728 \\
\hline 13.13888889 & 84.1696619 & 336.6786 & 841.6966 & 1851.7326 \\
\hline 13.16666667 & 84.3587665 & 337.4351 & 843.5877 & 1855.8929 \\
\hline 13.19444444 & 84.5478961 & 338.1916 & 845.479 & 1860.0537 \\
\hline 13.22222222 & 84.7370506 & 338.9482 & 847.3705 & 1864.2151 \\
\hline 13.25 & 84.92623 & 339.7049 & 849.2623 & 1868.3771 \\
\hline 13.27777778 & 85.1154342 & 340.4617 & 851.1543 & 1872.5396 \\
\hline 13.30555556 & 85.3046632 & 341.2187 & 853.0466 & 1876.7026 \\
\hline 13.33333333 & 85.493917 & 341.9757 & 854.9392 & 1880.8662 \\
\hline 13.36111111 & 85.6831955 & 342.7328 & 856.832 & 1885.0303 \\
\hline 13.38888889 & 85.8724986 & 343.49 & 858.725 & 1889.195 \\
\hline 13.41666667 & 86.0618263 & 344.2473 & 860.6183 & 1893.3602 \\
\hline 13.44444444 & 86.2511786 & 345.0047 & 862.5118 & 1897.5259 \\
\hline 13.47222222 & 86.4405553 & 345.7622 & 864.4056 & 1901.6922 \\
\hline 13.5 & 86.6299565 & 346.5198 & 866.2996 & 1905.859 \\
\hline 13.52777778 & 86.8193821 & 347.2775 & 868.1938 & 1910.0264 \\
\hline 13.55555556 & 87.0088321 & 348.0353 & 870.0883 & 1914.1943 \\
\hline 13.58333333 & 87.1983064 & 348.7932 & 871.9831 & 1918.3627 \\
\hline 13.61111111 & 87.387805 & 349.5512 & 873.878 & 1922.5317 \\
\hline
\end{tabular}




\begin{tabular}{|r|r|r|r|r|}
\hline 13.63888889 & 87.5773278 & 350.3093 & 875.7733 & 1926.7012 \\
\hline 13.66666667 & 87.7668747 & 351.0675 & 877.6687 & 1930.8712 \\
\hline 13.69444444 & 87.9564458 & 351.8258 & 879.5645 & 1935.0418 \\
\hline 13.72222222 & 88.146041 & 352.5842 & 881.4604 & 1939.2129 \\
\hline 13.75 & 88.3356601 & 353.3426 & 883.3566 & 1943.3845 \\
\hline 13.77777778 & 88.5253033 & 354.1012 & 885.253 & 1947.5567 \\
\hline 13.80555556 & 88.7149705 & 354.8599 & 887.1497 & 1951.7294 \\
\hline 13.83333333 & 88.9046615 & 355.6186 & 889.0466 & 1955.9026 \\
\hline 13.86111111 & 89.0943764 & 356.3775 & 890.9438 & 1960.0763 \\
\hline 13.88888889 & 89.2841151 & 357.1365 & 892.8412 & 1964.2505 \\
\hline
\end{tabular}

